\title{
Efficacy of antiviral therapies for COVID-19: a systematic review of randomized controlled trials
}

\author{
Charan Thej Reddy Vegivinti ${ }^{1}$, Kirk W. Evanson ${ }^{2}$, Hannah Lyons ${ }^{3,4}$, Izzet Akosman ${ }^{3,10}$, Averi Barrett ${ }^{3}$, \\ Nicole Hardy ${ }^{3}$, Bernadette Kane ${ }^{2}$, Praneeth Reddy Keesari ${ }^{5}$, Yashwitha Sai Pulakurthi ${ }^{5}$, Erin Sheffels ${ }^{2 *}$, \\ Prasanth Balasubramanian ${ }^{1}$, Richa Chibbar 6 , Spandana Chittajallu ${ }^{7}$ Kathryn Cowie ${ }^{3}$, J. Karon $^{3}$, Lauren Siegel ${ }^{3}$, \\ Ranita Tarchand ${ }^{3}$, Caleb Zinn ${ }^{3}$, Nitin Gupta ${ }^{8,9}$, Kevin M. Kallmes ${ }^{3}$, Kavitha Saravu ${ }^{8,9}$ and Jillienne Touchette ${ }^{2}$
}

\begin{abstract}
Background: Coronavirus disease 2019 (COVID-19) continues to pose a significant threat to public health worldwide. The purpose of this study was to review current evidence obtained from randomized clinical trials on the efficacy of antivirals for COVID-19 treatment.

Methods: A systematic literature search was performed using PubMed to identify randomized controlled trials published up to September 4, 2021 that examined the efficacy of antivirals for COVID-19 treatment. Studies that were not randomized controlled trials or that did not include treatment of COVID-19 with approved antivirals were excluded. Risk of bias was assessed using the Scottish Intercollegiate Guidelines Network (SIGN) method. Due to study heterogeneity, inferential statistics were not performed and data were expressed as descriptive statistics.
\end{abstract}

Results: Of the 2,284 articles retrieved, 31 (12,440 patients) articles were included. Overall, antivirals were more effective when administered early in the disease course. No antiviral treatment demonstrated efficacy at reducing COVID19 mortality. Sofosbuvir/daclatasvir results suggested clinical improvement, although statistical power was low. Remdesivir exhibited efficacy in reducing time to recovery, but results were inconsistent across trials.

Conclusions: Although select antivirals have exhibited efficacy to improve clinical outcomes in COVID-19 patients, none demonstrated efficacy in reducing mortality. Larger RCTs are needed to conclusively establish efficacy.

Keywords: Systematic review, COVID-19, Antiviral, SARS-CoV-2, Therapeutic, Randomized controlled trial

\section{Background}

Coronavirus disease 2019 (COVID-19) continues to present a significant challenge to healthcare systems worldwide, with approximately 269 million confirmed cases of the disease that have led to 5.3 million deaths as of December 12, 2021 [1]. COVID-19 develops from a viral

\footnotetext{
*Correspondence: erinsheffels@supedit.com

2 Superior Medical Experts, 1425 Minnehaha Ave E, P.O. Box 6000545, St Paul, MN 55106, USA

Full list of author information is available at the end of the article
}

infection, severe acute respiratory syndrome coronavirus 2 (SARS-CoV-2), which can elicit exaggerated immune and inflammatory responses if the infection progresses [2]. As such, there are a wide variety of therapeutic strategies that have been used to treat the disease at various stages, including antiviral, antiretroviral, antimalarial, anti-inflammatory, corticosteroid, immunomodulatory, and immunoglobulin therapies [3].

Research on drug therapies for COVID-19 has relied heavily on results obtained from observational studies, many of which contain biases resulting from 
demographical differences, patient/disease heterogeneity, differences in institutional practices and standards, and differences in healthcare infrastructure and financial support. As a result of the substantial heterogeneity across studies, a consensus on COVID-19 therapies has remained elusive.

Antiviral drugs, such as remdesivir, represent promising drug candidates to attenuate viral and disease progression. Although there have been comprehensive presentations of outcomes associated with antiviral treatments for COVID-19 obtained from randomized controlled design, the number of relevant randomized controlled trials were limited in these studies because they were either published early in the pandemic [4] or had search dates that ended during the middle of the pandemic [5] and many new trails have been published in the past year. Additionally, while a more recent review has been published, it did not include a description of how the study was carried out and was not PRISMA compliant [6]. Here, we conducted a systematic review of RCTs that examined antiviral efficacy for COVID-19 treatment.

\section{Methods}

\section{Literature search}

A systematic literature search was conducted to identify RCTs that investigated antiviral treatments of COVID-19 using PubMed through Nested Knowledge, an AutoLit platform for living systematic reviews [7]. The search terms used are listed in Table 1, and search filters or limits were not used. All fields were searched and the search was not limited to title/abstract. Databases used included Embase, PubMed, PubMed Central, and Web of Science. This study was conducted in accordance with the Preferred Reporting Items for Systematic Reviews and MetaAnalyses (PRISMA) guidelines [8]. A review protocol was created by the authors in order to establish the framework for this systematic review and can be viewed on the Nested Knowledge platform [9]. Concepts outlined in the protocol were then developed into a custom tagging hierarchy in order to tag each study, which reflected specific evidence underneath the categories we laid out. For example, under outcomes, there is a node for Clinical Improvement that reflects an outcome we intended to gather from each study. Tagging of full-text articles was completed in order to trace concepts and link qualitative synthesis. The review was not registered.

\section{Study selection and quality assessment}

Studies published between November 1, 2019 and September 4, 2021 were considered. Prior to screening, all studies published before November 1, 2019 or not published in English were automatically excluded by Nested Knowledge. Additionally, during the screening process, a machine learning algorithm ordered studies based on what was most likely to be included, and the software automatically de-duplicated studies. No further automation was used, as each article was screened by one of nine contributors and inclusion was independently verified by one author $(\mathrm{NH})$. All studies that used a randomized controlled design to examine clinical outcomes related to antiviral treatment of COVID-19 were included. Only drugs approved for use as antivirals were considered, including baloxavir marboxil [10], lopinavir/ ritonavir (LPV/r) [11], atazanavir [12], sofosbuvir [13], daclatasvir [14], remdesivir [15], ribavirin [16], favipiravir [17], umifenovir (Arbidol) [18], and azvudine [19] and novaferon [20]. The following article types were excluded: observational, editorial, opinion, in vitro or in vivo study, review, methods, case series or report, guidelines, and articles that were not published in English.

\section{Data collection}

Data was manually extracted through the Nested Knowledge platform for living systematic reviews by one of 11 contributors and independently checked for accuracy by one author for each study. Tags from the custommade Nested Knowledge tagging hierarchy were preconfigured as data elements in order to keep variables organized. Variables in the platform were classified as continuous, categorical, or dichotomous, and manually extracting data from full-text articles facilitated statistical analysis and qualitative synthesis. When available, background characteristics were collected, including age, sex, time from symptom onset to the start of treatment, white blood cell count (WBC), and oxygen saturation $\left(\mathrm{SpO}_{2}\right)$. Intervention-related information, such as doses and regiment, follow-up period, and concomitant medications, were also collected. The outcomes collected included mortality, incidence of mechanical ventilation and intensive care unit (ICU) admission, number of patients with negative reverse transcription polymerase chain reaction (RT-PCR) tests, duration of hospitalization, incidence of clinical improvement, and improvement in $\mathrm{SpO}_{2}$.

\section{Risk of bias and statistical analysis}

Risk of bias was assessed using the Scottish Intercollegiate Guidelines Network (SIGN) checklist for randomized controlled trials [21]. Items that are considered in the SIGN checklist include an appropriate and clearly focused question, randomized assignment, adequate concealment, blinding, similar treatment and control groups at the start of the trial, the treatment is the only difference between groups, standard outcome measurement, percentage of subjects that dropped, intention to treat analysis, comparable results for all sites, and overall assessment of the study. The grading system includes 
Table 1 Search terms

\begin{tabular}{|c|c|c|c|}
\hline Search terms & Database & Search date & $\begin{array}{l}\text { Number } \\
\text { of results }\end{array}$ \\
\hline $\begin{array}{l}\text { (Lopinavir OR Ritonavir OR Remdesivir OR Ribavirin OR Arbidol OR Favipiravir OR Sofosbuvir OR } \\
\text { Daclatasvir) AND (COVID-19 OR SARS-COV-2 OR "novel coronavirus") AND (RCT OR "randomized con- } \\
\text { trolled trial" OR "randomised controlled trial" OR "randomized" OR "randomised") }\end{array}$ & Web of Science & $12-01-2021$ & 336 \\
\hline $\begin{array}{l}\text { (Lopinavir OR Ritonavir OR Remdesivir OR Ribavirin OR Arbidol OR Favipiravir OR Sofosbuvir OR } \\
\text { Daclatasvir) AND (COVID-19 OR SARS-COV-2 OR "novel coronavirus") AND (RCT OR "randomized con- } \\
\text { trolled trial" OR "randomised controlled trial" OR "randomized" OR "randomised") }\end{array}$ & Embase & $12-01-2021$ & 25 \\
\hline $\begin{array}{l}\text { (Lopinavir OR Ritonavir OR Remdesivir OR Ribavirin OR Arbidol OR Favipiravir OR Sofosbuvir OR } \\
\text { Daclatasvir) AND (COVID-19 OR SARS-COV-2 OR "novel coronavirus") AND (RCT OR "randomized con- } \\
\text { trolled trial" OR "randomised controlled trial" OR "randomized" OR "randomised") }\end{array}$ & PubMed & $12-01-2021$ & 339 \\
\hline $\begin{array}{l}\text { (Lopinavir OR Ritonavir OR Remdesivir OR Ribavirin OR Arbidol OR Favipiravir OR Sofosbuvir OR Daclatasvir } \\
\text { OR Ivermectin OR Azithromycin) AND (COVID-19 OR SARS-COV-2 OR "novel coronavirus") AND (RCT OR } \\
\text { "randomized controlled trial") }\end{array}$ & PubMed & $12-04-2021$ & 162 \\
\hline $\begin{array}{l}\text { ("Therapeutics" OR "antiviral therapies") AND (RCT OR "randomized controlled trial") AND (COVID-19 OR } \\
\text { SAR-COV-2 OR "coronavirus") }\end{array}$ & PubMed & $1-04-2021$ & 47 \\
\hline $\begin{array}{l}\text { ("randomized controlled trial" OR RCT) AND (Ribavirin) AND (COVID-19 OR SARs-CoV-2 OR "coronavirus" } \\
\text { OR SAR-COV-2) }\end{array}$ & PubMed & $12-04-2021$ & 14 \\
\hline $\begin{array}{l}\text { (SARs-CoV-2 OR SARs OR COVID-19 OR "coronavirus") AND (LPV/RTV OR Lopinavir OR Ritonavir) AND (RCT } \\
\text { or "randomized controlled trial") }\end{array}$ & PubMed & $12-05-2021$ & 68 \\
\hline $\begin{array}{l}\text { (Sofosbuvir OR Daclatasvir) AND (RCT OR "randomized controlled trial") AND (COVID-19 OR SAR-COV-2 OR } \\
\text { "novel coronavirus") }\end{array}$ & PubMed & $12-04-2021$ & 7 \\
\hline $\begin{array}{l}\text { SARs-CoV-2 OR SARs OR COVID-19 OR "coronavirus" OR covid AND ("antiviral drugs") AND (RCT OR "rand- } \\
\text { omized controlled trial" OR "randomised controlled trial") }\end{array}$ & PubMed & $12-04-2021$ & 14 \\
\hline $\begin{array}{l}\text { ("antiviral therapies" OR "antiviral drugs") AND (RCT OR "randomized controlled trial" OR "randomised } \\
\text { controlled trial") AND (COVID-19 OR SAR-COV-2 OR coronavirus OR covid) }\end{array}$ & PubMed & $12-04-2021$ & 20 \\
\hline $\begin{array}{l}\text { (SARs-CoV-2 OR SARs OR COVID-19 OR "coronavirus" OR "covid") AND (LPV/RTV OR Lopinavir OR Ritonavir) } \\
\text { AND ("randomised controlled trial" OR RCT or "randomized controlled trial") }\end{array}$ & PubMed & $12-04-2021$ & 73 \\
\hline $\begin{array}{l}\text { (Lopinavir OR Ritonavir OR Remdesivir OR Ribavirin OR Arbidol OR Favipiravir OR Sofosbuvir OR } \\
\text { Daclatasvir) AND (COVID-19 OR SARS-COV-2 OR "novel coronavirus" OR covid) AND (RCT OR "randomized } \\
\text { controlled trial" OR "randomised controlled trial")) }\end{array}$ & PubMed & $12-04-2021$ & 124 \\
\hline $\begin{array}{l}\text { ("novel coronavirus" OR COVID-19 OR SARS-CoV-2) AND (RCT OR "randomized controlled trial") AND } \\
\text { ("antiviral therapy") AND (Lopinavir OR Ritonavir OR Remdesivir OR Ribaviron OR Arbidol OR Favipiravir OR } \\
\text { Daclatasvir OR Sofosbuvir) }\end{array}$ & PubMed & $12-04-2021$ & 11 \\
\hline $\begin{array}{l}\text { (Lopinavir OR Ritonavir OR Remdesivir OR Ribavirin OR Arbidol OR Favipiravir OR Sofosbuvir OR } \\
\text { Daclatasvir) AND (COVID-19 OR SARS-COV-2 OR "novel coronavirus") AND (RCT OR "randomized con- } \\
\text { trolled trial") }\end{array}$ & PubMed Central & $02-08-2021$ & 1971 \\
\hline $\begin{array}{l}\text { (Lopinavir OR Ritonavir OR Remdesivir OR Ribavirin OR Arbidol OR Favipiravir OR Sofosbuvir OR } \\
\text { Daclatasvir) AND (COVID-19 OR SARS-COV-2 OR "novel coronavirus") AND (RCT OR "randomized con- } \\
\text { trolled trial") }\end{array}$ & PubMed & $12-04-2021$ & 124 \\
\hline ("COVID-19" OR "coronavirus" OR SAR-COV-2) AND ("Ribavirin") AND (RCT OR "randomized control trial") & PubMed & $12-04-2021$ & 5 \\
\hline
\end{tabular}

levels of evidence rated from $1++$ high quality to 2 - high risk of bias, as well as grades of recommendation, followed by grades of recommendation from grade A to D. Two independent reviewers assessed each study. Assessments were verified and disagreements were adjudicated by a third reviewer. Due to heterogeneity in treatments used and outcomes reported, inferential statistics were not performed, and data were expressed as descriptive statistics only. Continuous data were reported as mean \pm standard deviation (SD) or median (interquartile range $[\mathrm{IQR}])$ unless otherwise noted.

\section{Results}

A total of 2,284 articles were identified from the search terms, of which 31 studies that included 12,440 patients used randomized controlled designs to examine the efficacy of antiviral therapy on COVID-19 [22-53]. A PRISMA diagram detailing the search strategy is shown in Fig. 1. Of the articles identified, 30 were excluded after fulltext review [54-83]. One study was originally included, but was later retracted due to concerns about data integrity, and thus was excluded [42]. Antiviral treatments compared in the included studies were umifenovir (Arbidol) [25, 29, 31, 47], baloxavir marboxil [30], enisamium [50], 


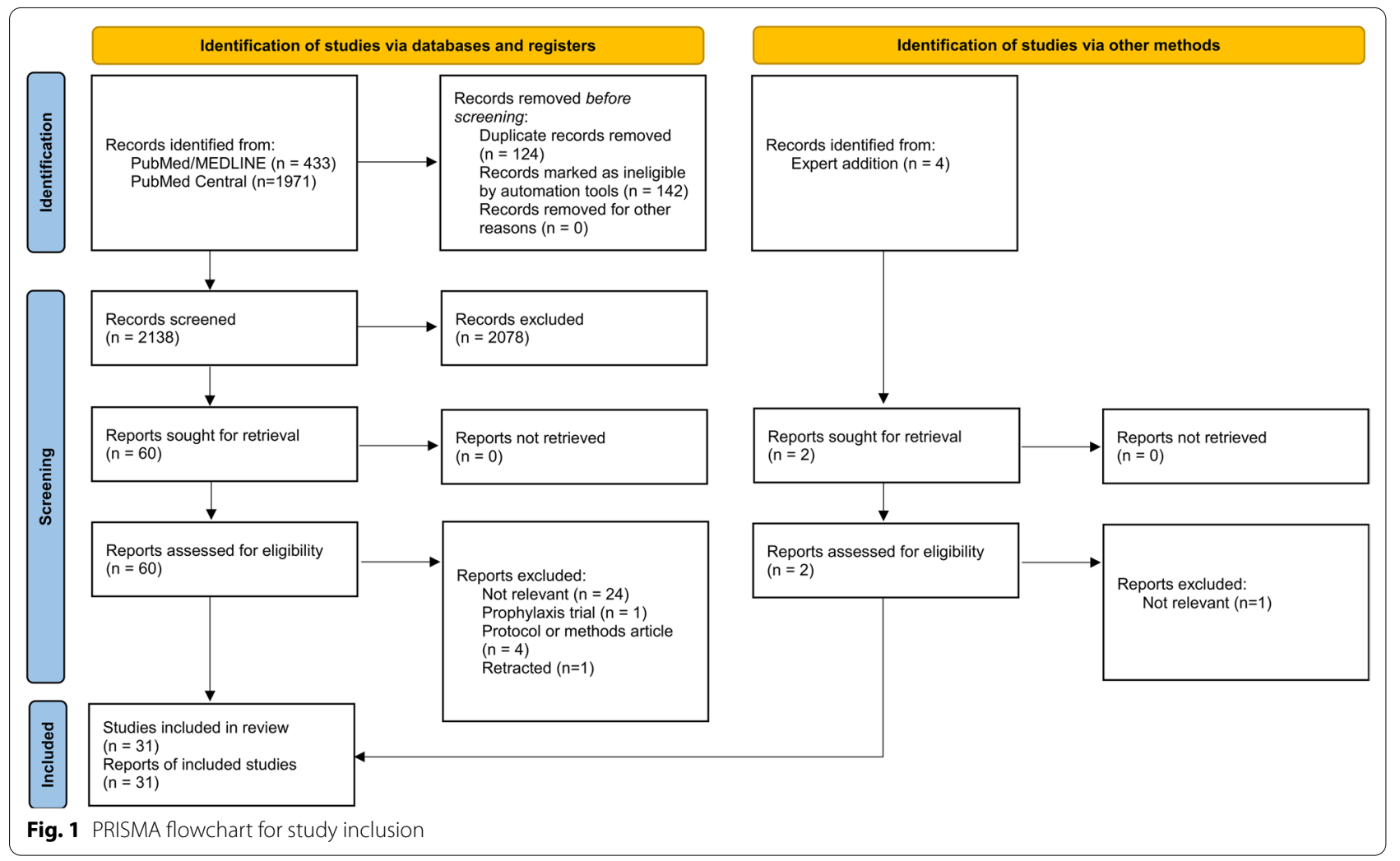

favipiravir [25, 30, 35, 40-42, 44, 45, 48, 52], lopinavir/ritonavir (LPV/r) [24, 26, 27, 29, 31, 37, 38, 44, 47], remdesivir [23, 34, 36, 39, 51, 53], ribavirin [22], sofosbuvir/daclatasvir [22, 32, 33, 46, 49], sofosbuvir/ledipasvir [28], sofosbuvir/ravidasvir [46], and sofosbuvir/velpatasvir [43]. The study characteristics and baseline patient characteristics are summarized in Table 2. The outcomes of interest and study conclusions are summarized in Table 3. Two studies were rated low quality on the risk of bias assessment, with bias favoring the test treatment $[49,51]$. The remaining studies were rated either acceptable or high quality (Additional file 1).

\section{Favipiravir}

Favipiravir is an antiviral used to treat influenza in Japan. It is a purine analog that inhibits viral RNA-dependent RNA polymerase, blocking viral genome replication and transcription [84]. We identified nine RCTs that examined the efficacy of favipiravir in treating COVID-19. Five trials found significant differences between the favipiravir treatment and comparator groups [35, 45, 48, 52, 85] and four did not find significant differences [30, 40, 41, 44] (Table 3).

Zhao et al. conducted a multicentric open-label trial that compared favipiravir with a control group [45]. Patients were randomly assigned to receive favipiravir or treatments other than favipiravir, chosen at the discretion of the treating physician. Patients treated with favipiravir had a significantly shorter median time to positive-to-negative RT-PCR SARS-CoV-2 test conversion (17 days) compared to the control group (26 days; hazard ratio [HR]: 2.1 [95\% confidence interval [CI] 1.1-4.0], $\mathrm{p}=0.038$ ). The trial ended after 30 days, at which time the favipiravir group had a significantly higher incidence of conversion to negative RT-PCR tests $(80.6 \%$ [29/36]) compared to the control group (52.6\% [10/19], $\mathrm{p}=0.030)$. Mortality did not occur in either group within the 30-day study period.

Shinkai et al. investigated the efficacy of favipiravir in COVID-19 patients without oxygen therapy in a singleblind, placebo-controlled trial [52]. Patients received favipiravir or a placebo on the same schedule. They defined clinical improvement by four clinical parameters: temperature, oxygen saturation, chest imaging findings, and viral clearance assessed with RT-PCR. Patients treated with favipiravir met the criteria for clinical improvement significantly earlier (11.9 days [95\% CI: 10.0-13.1 days]) than patients in the placebo group (14.7 days [95\% CI: $10.5-17.9$ days], $\mathrm{p}=0.014)$. The difference in time to improvement was also significant in the covariateadjusted Cox proportional hazards model (HR: 1.59 [95\% CI 1.02-2.48]). Within the individual parameters, time to 


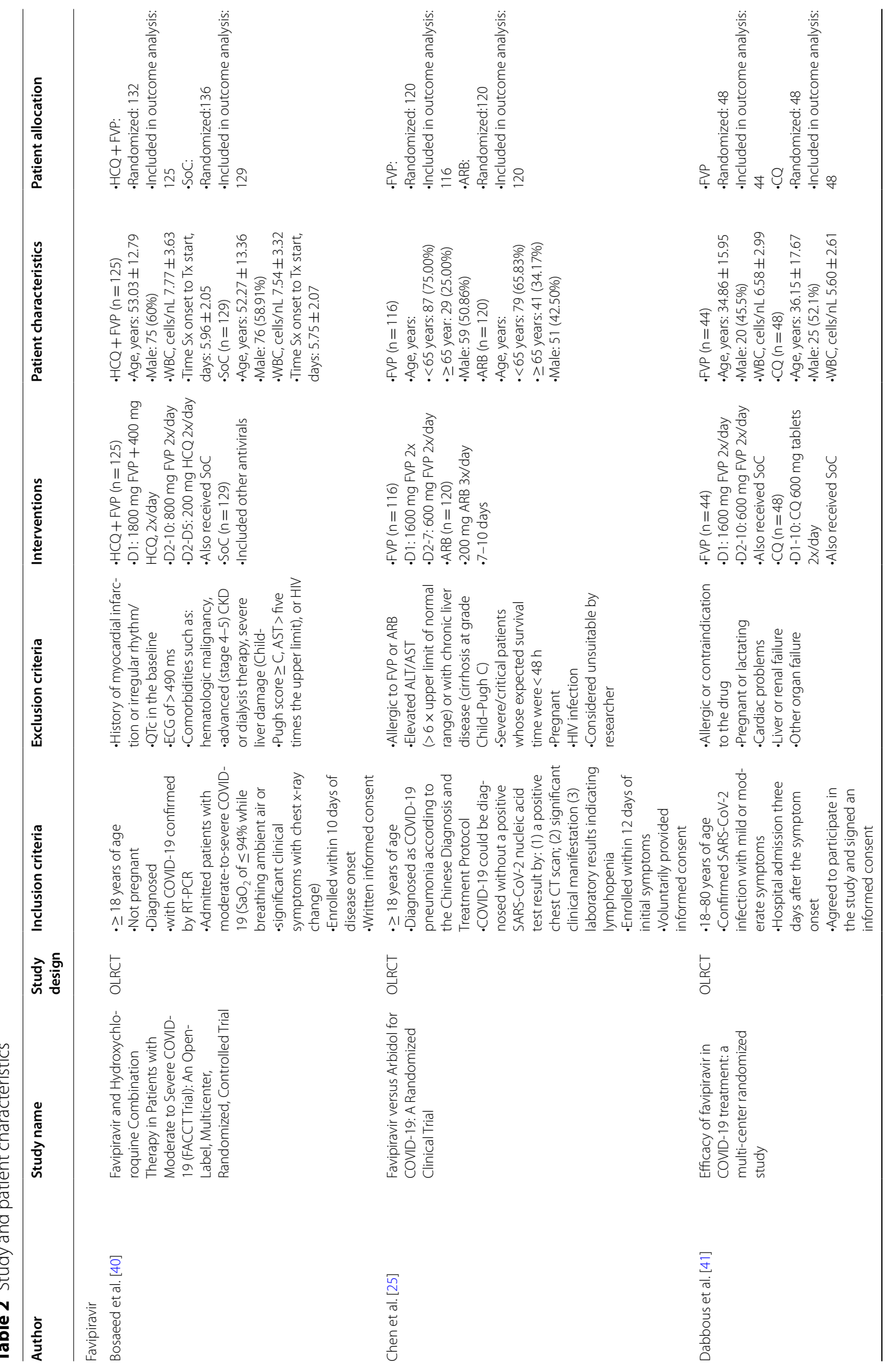




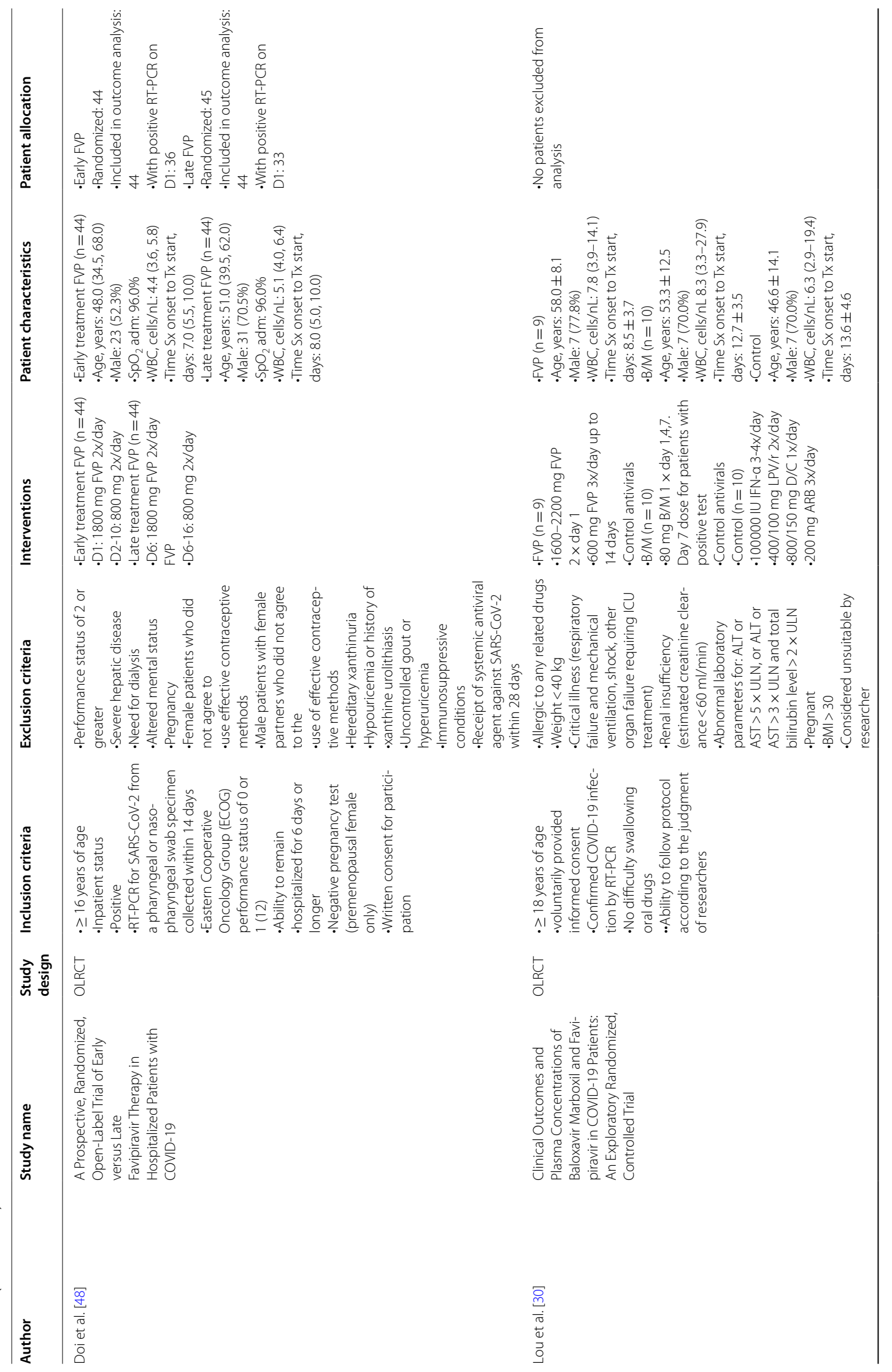




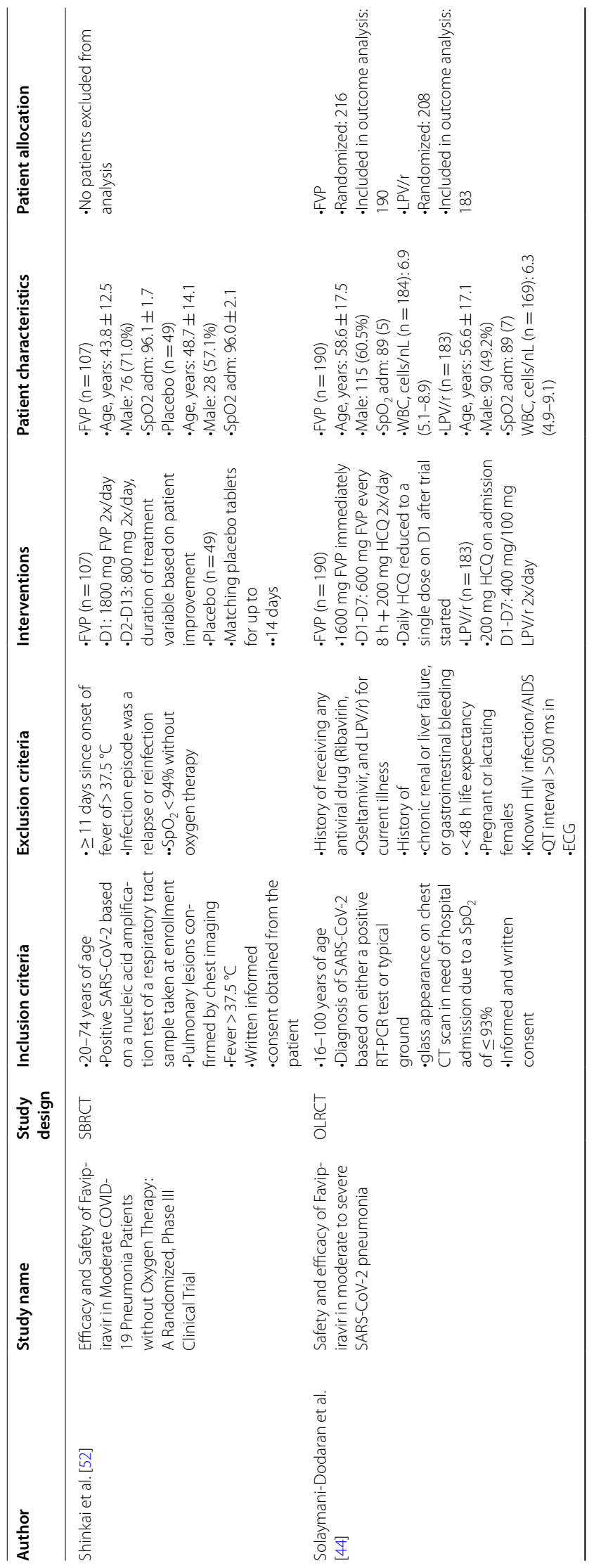




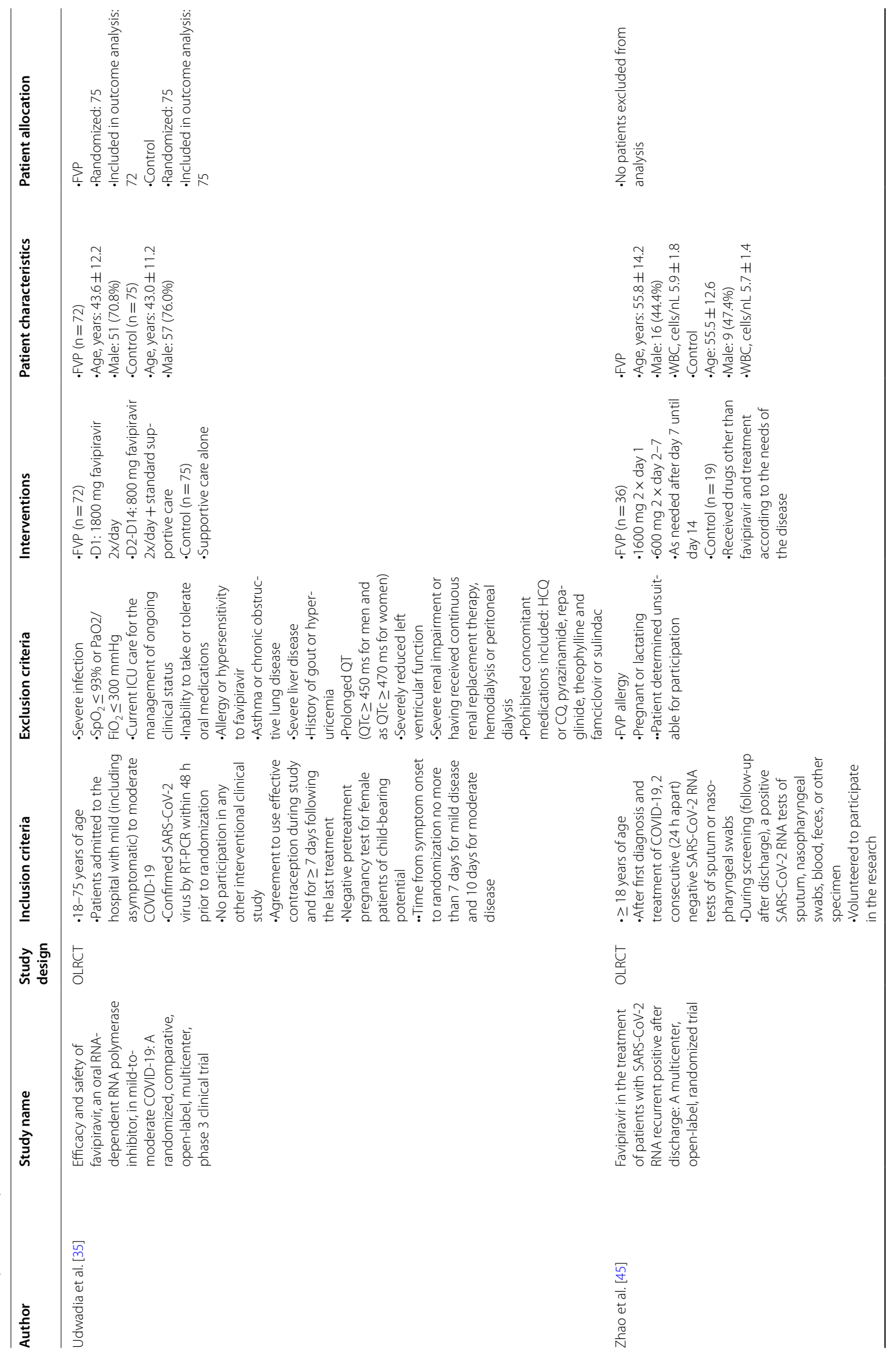




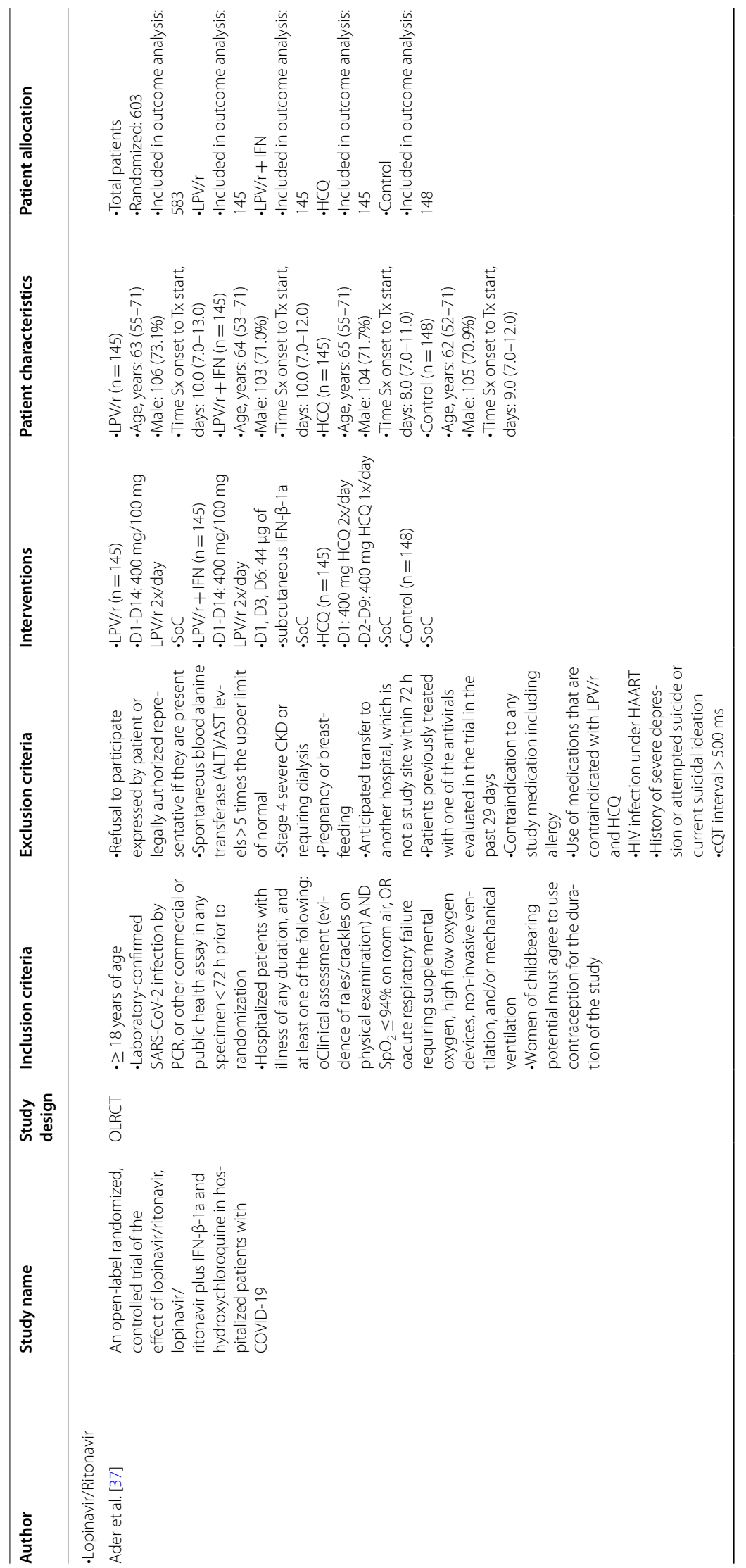




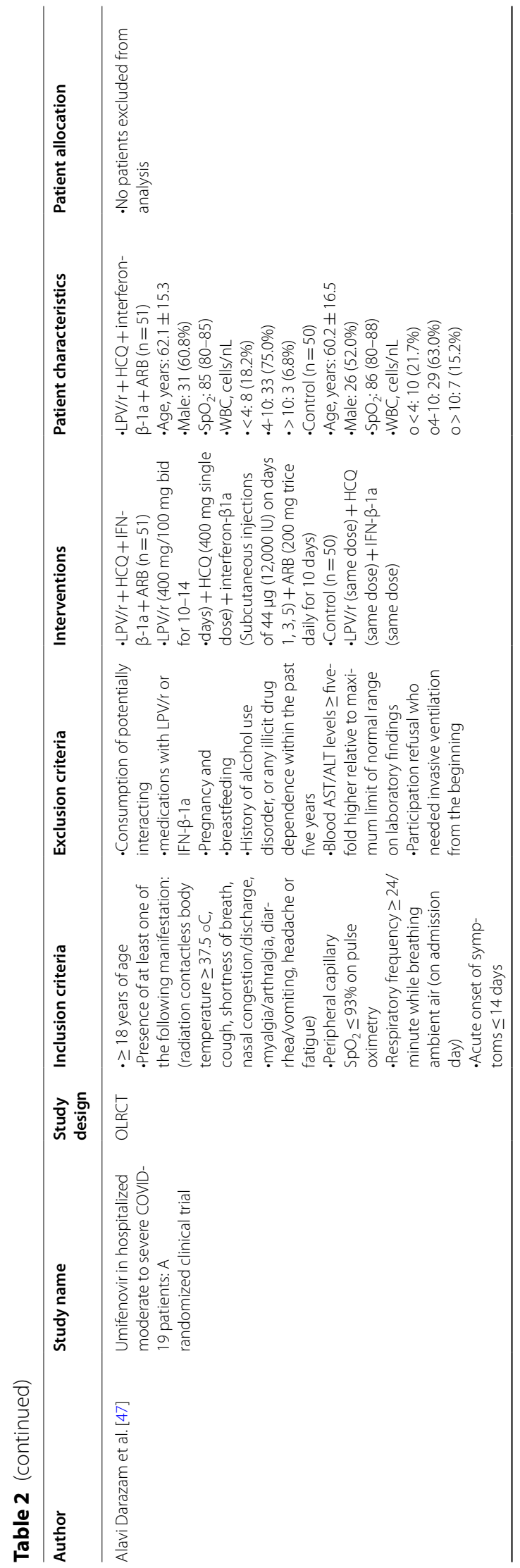




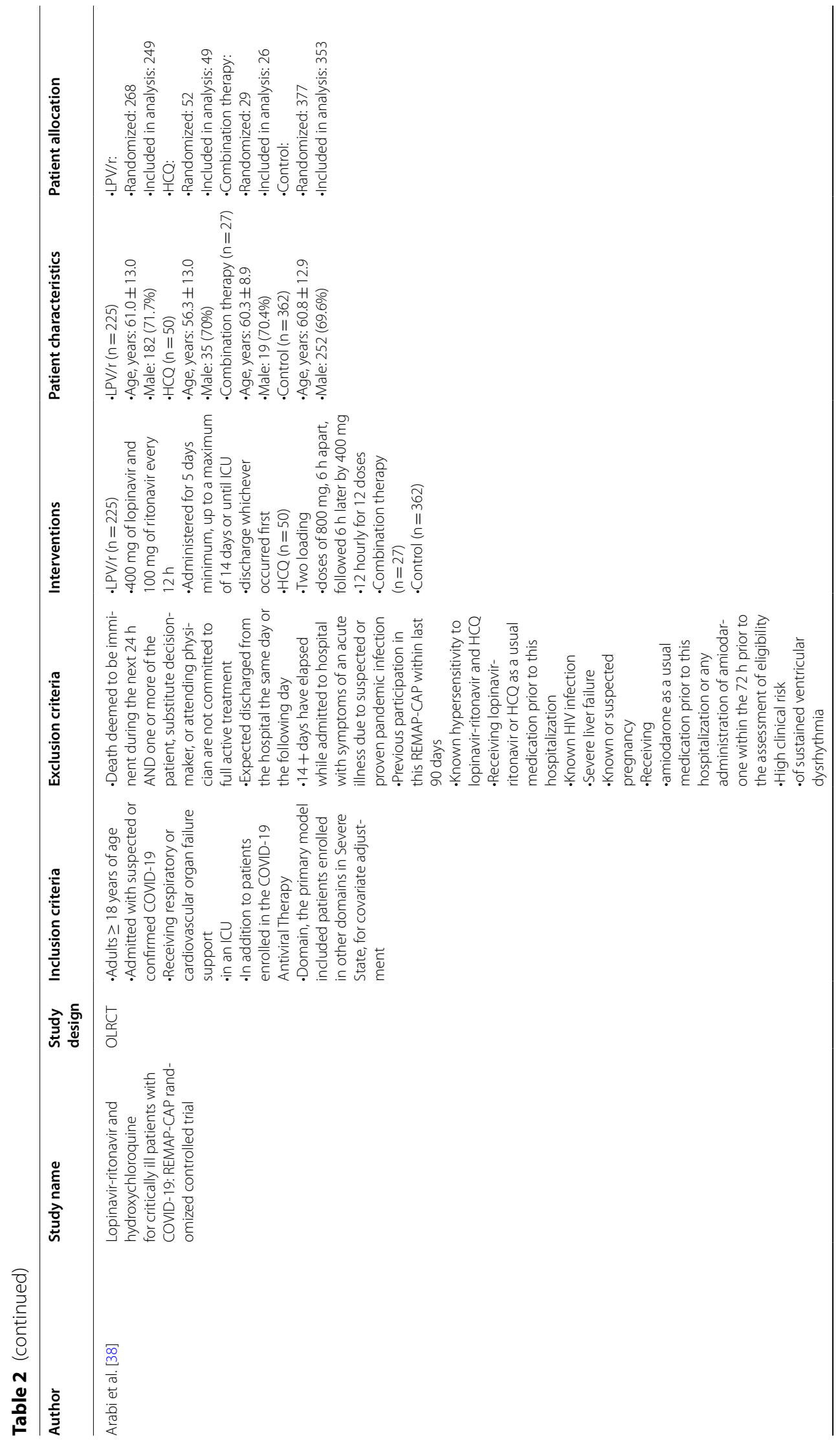




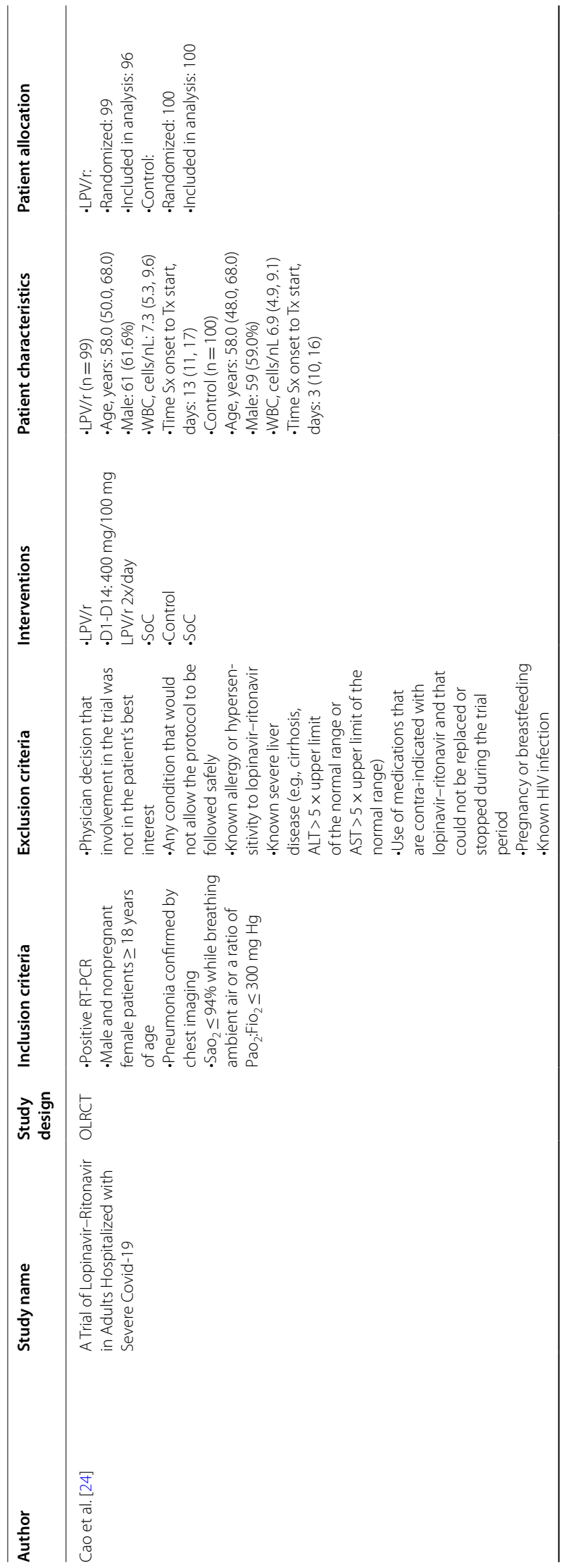




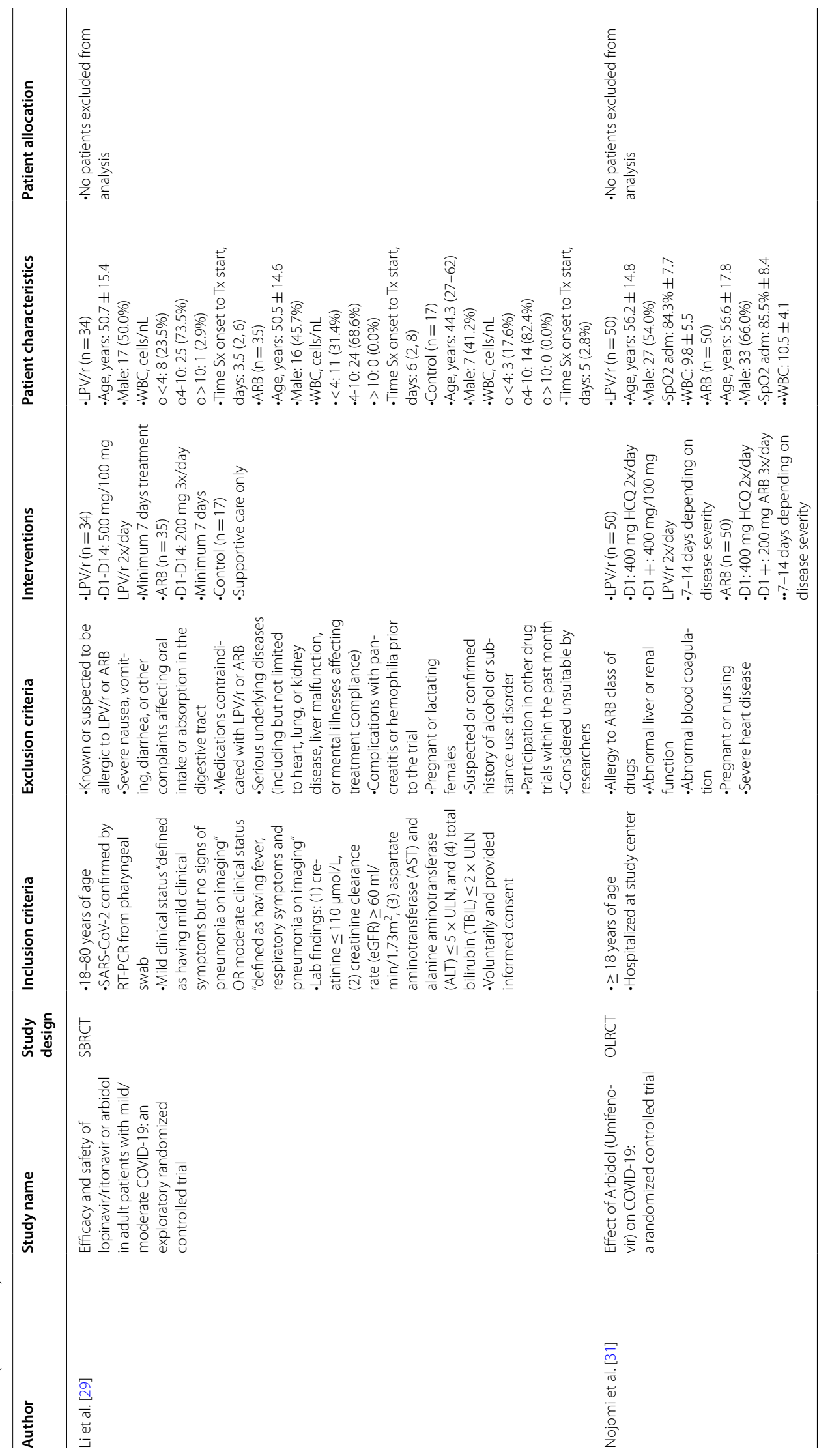




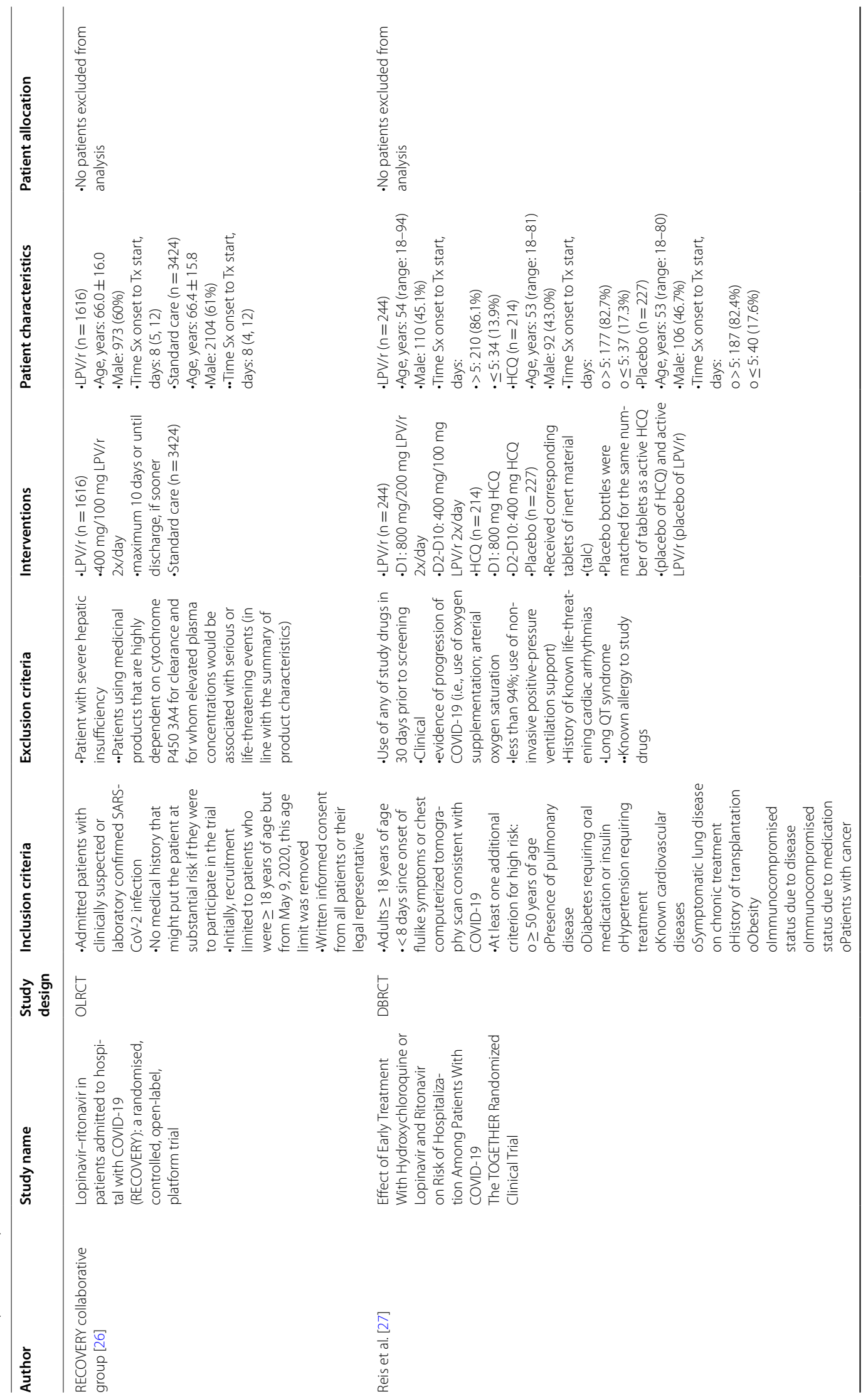




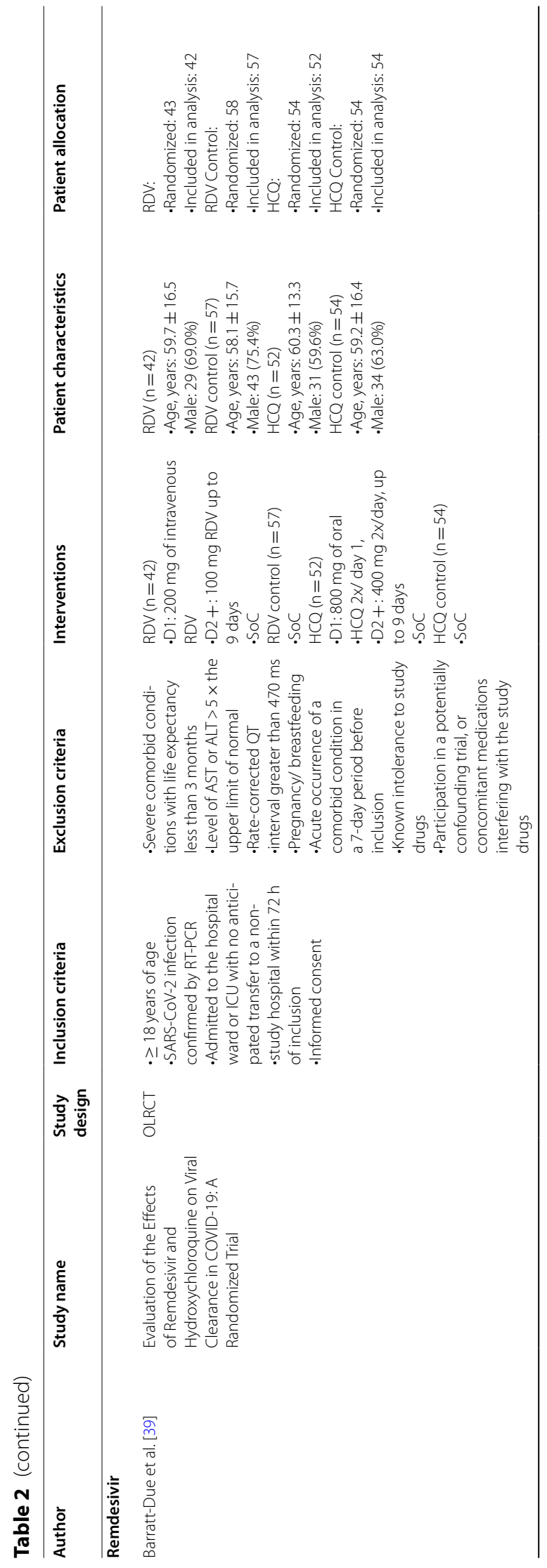




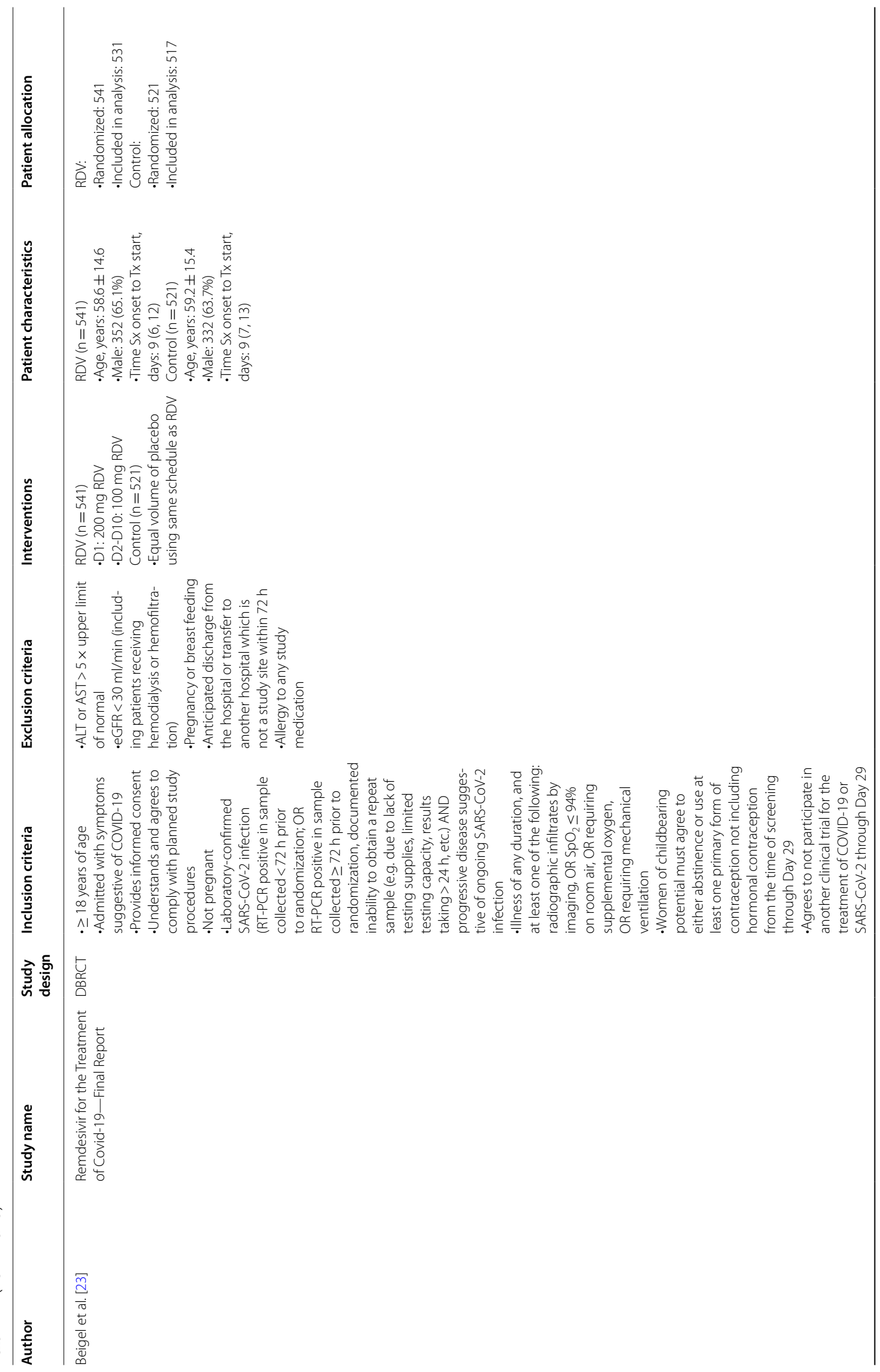




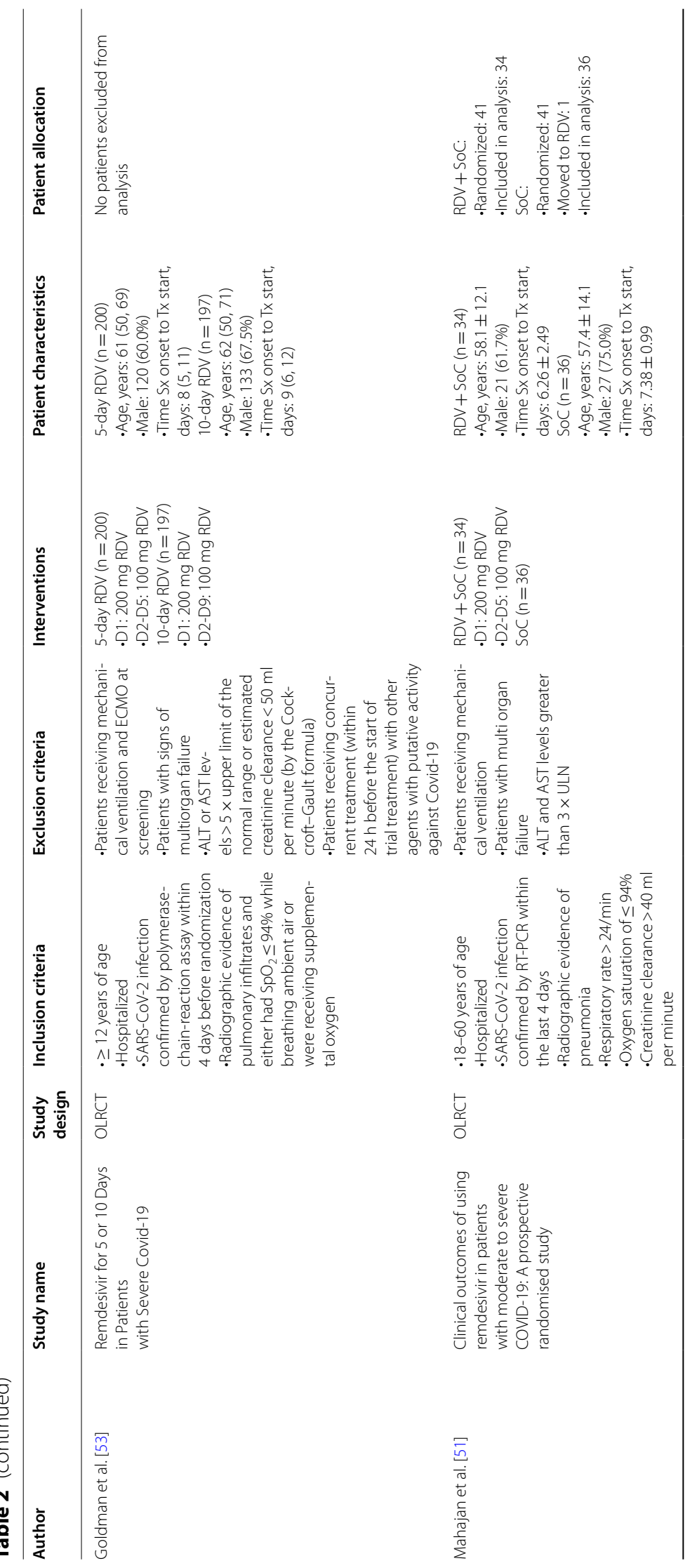




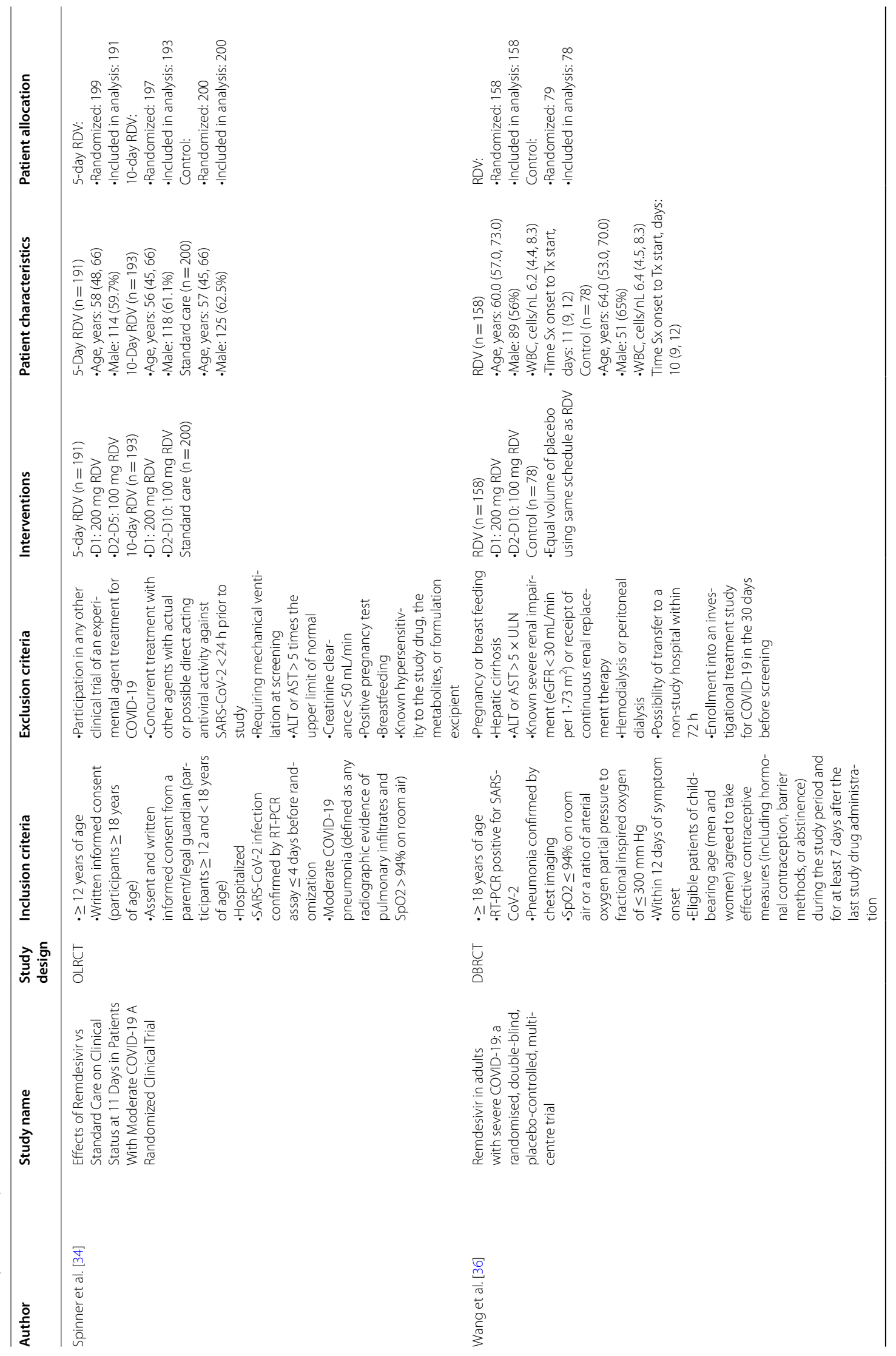




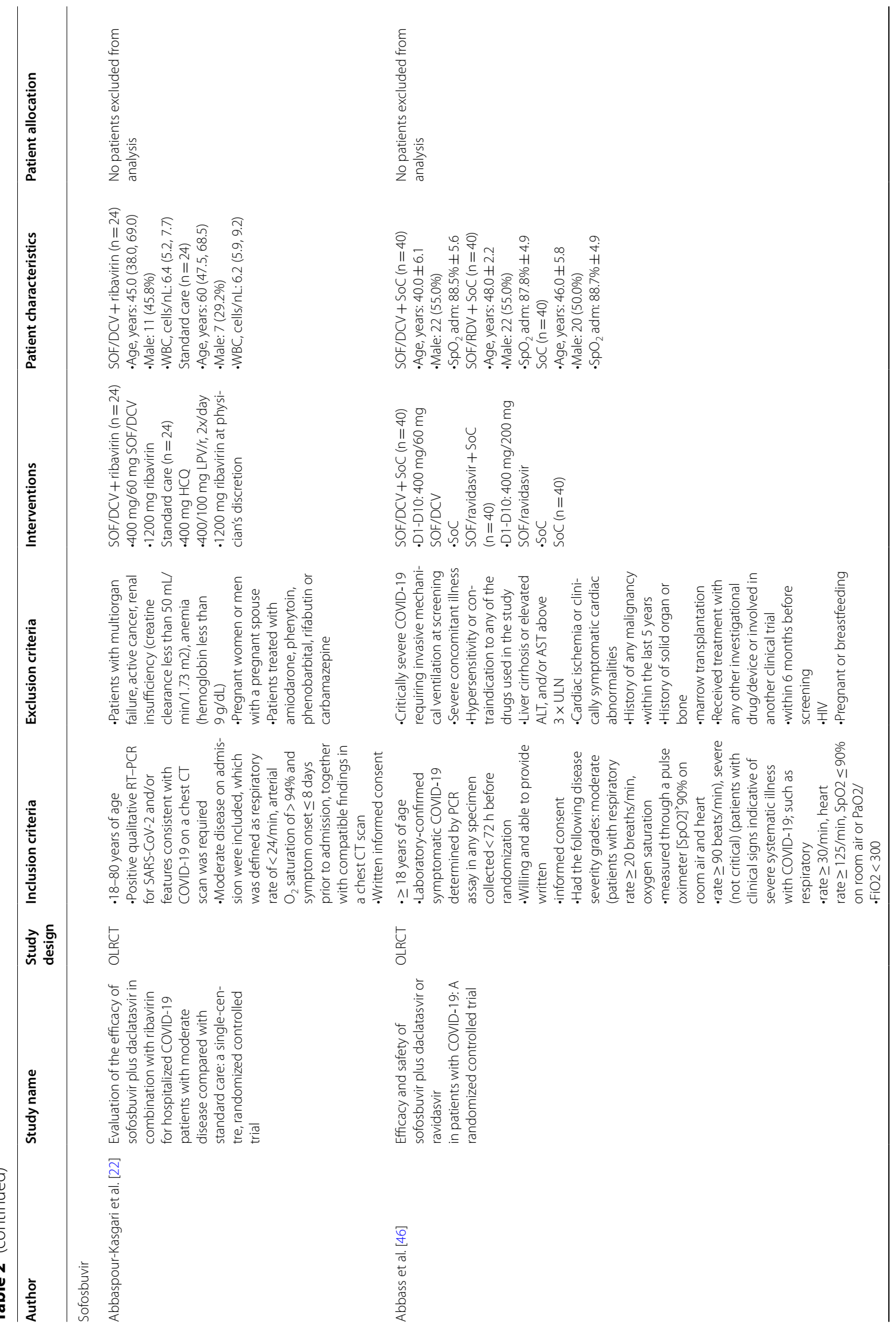




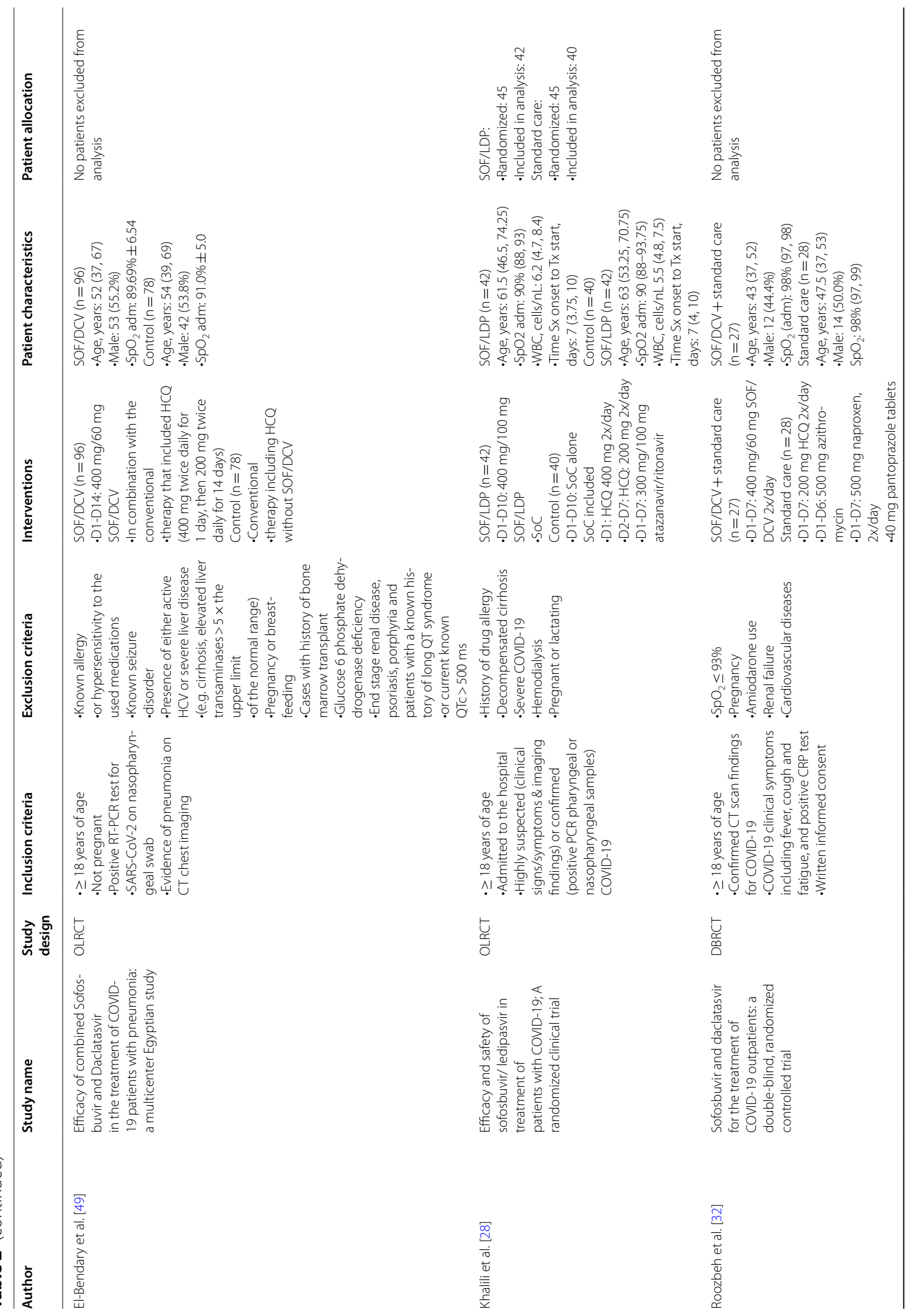




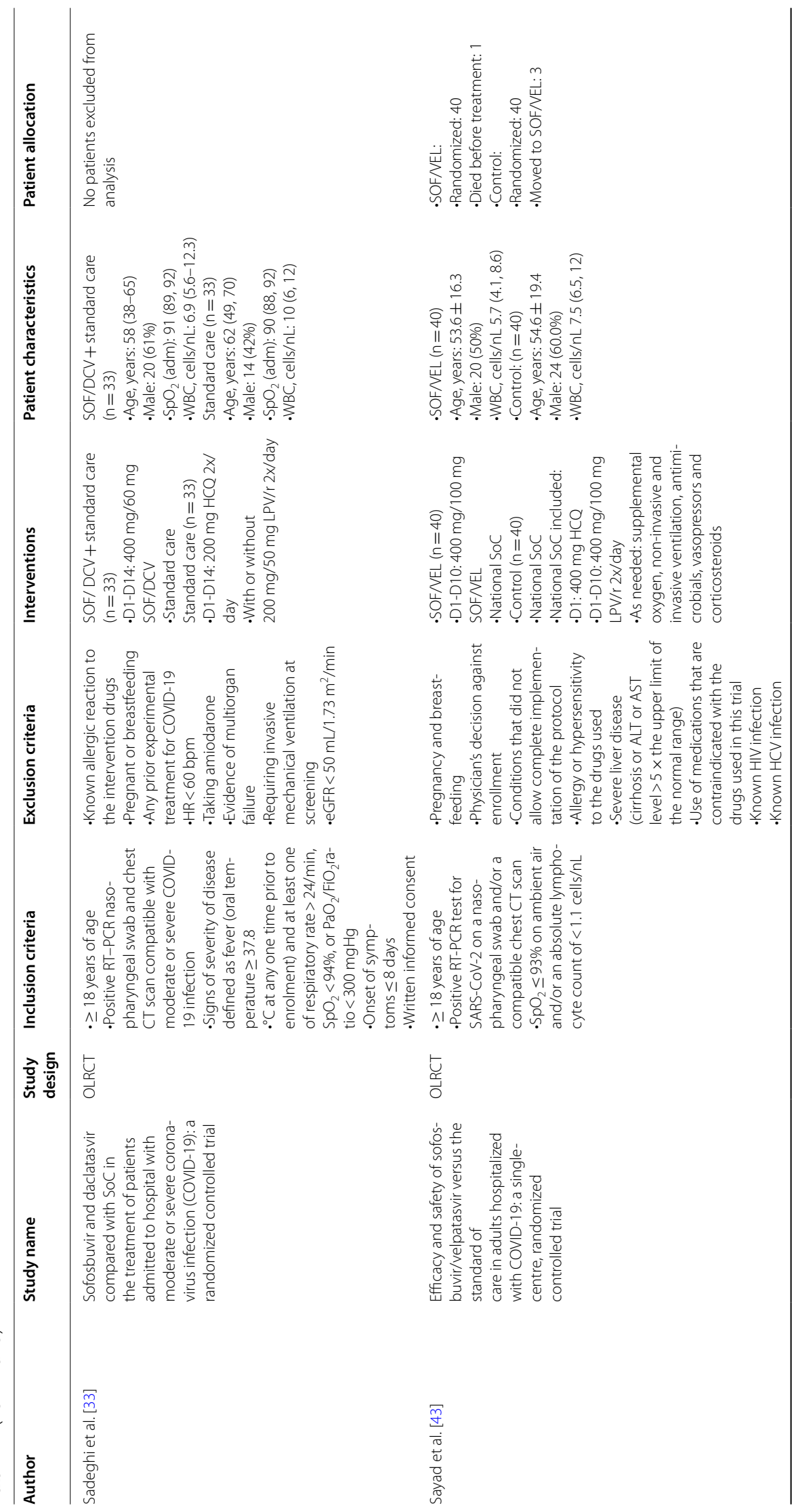




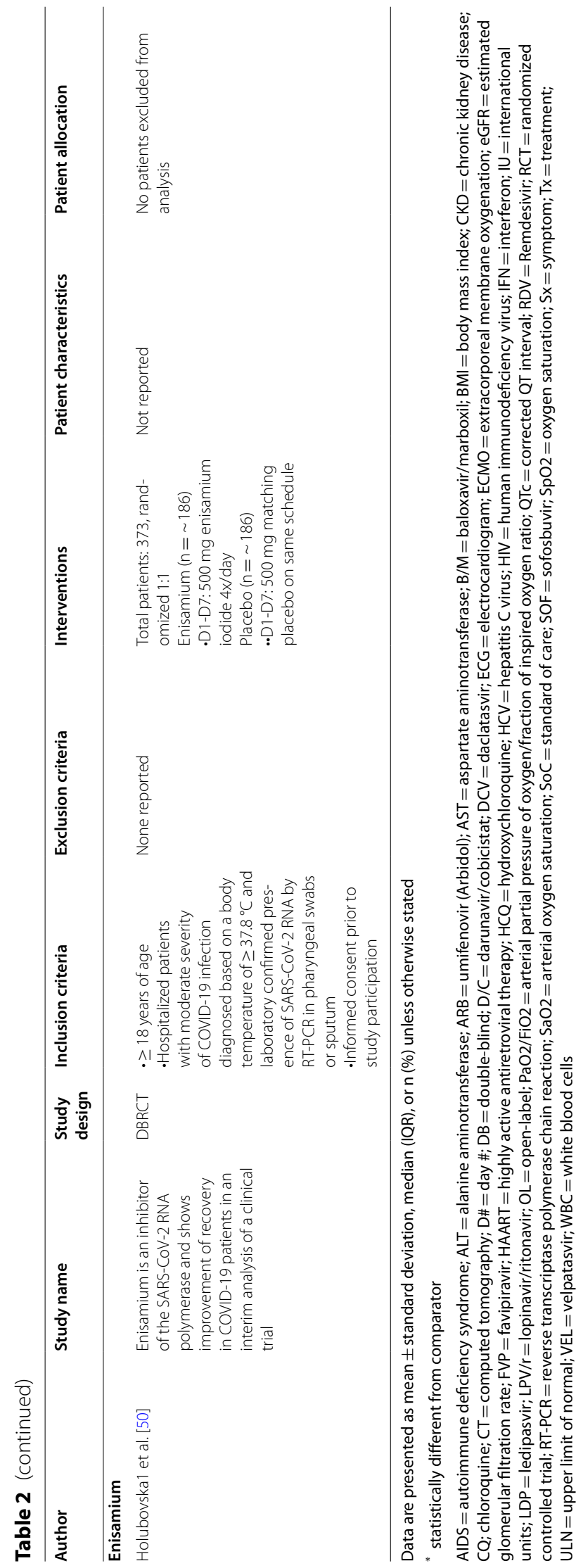


Table 3 Patient Outcomes

\begin{tabular}{|c|c|c|c|c|c|c|}
\hline Author & Study name & Primary endpoint & Primary outcomes & Other outcomes & Limitations & Interpretation \\
\hline $\begin{array}{l}\text { Bosaeed } \\
\text { et al. [40] }\end{array}$ & $\begin{array}{l}\text { Favipiravir and } \\
\text { Hydroxychloro- } \\
\text { quine Combina- } \\
\text { tion } \\
\text { Therapy in } \\
\text { Patients with } \\
\text { Moderate to } \\
\text { Severe COVID- } \\
19 \text { (FACCT Trial): } \\
\text { An Open-Label, } \\
\text { Multicenter, } \\
\text { Randomized, } \\
\text { Controlled Trial }\end{array}$ & $\begin{array}{l}\text {-Time to clinical } \\
\text {-improvement } \\
\text {-Defined as } \\
\text { the time from } \\
\text { randomization to } \\
\text { an improvement } \\
\text { of two points on } \\
\text { a seven-category } \\
\text { ordinal scale or live } \\
\text { discharge from the } \\
\text { hospital, which- } \\
\text { ever came first }\end{array}$ & $\begin{array}{l}\text { HCQ + FVP }(n=125) \\
\text {-Time to clinical improvement, days: } 9(8,12) \\
\text { SoC }(n=129) \\
\text {-Time to clinical improvement, days: } 7(6,10)\end{array}$ & 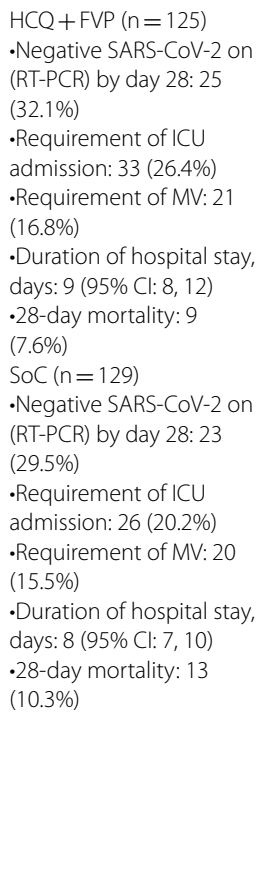 & $\begin{array}{l}\text {-Open-label } \\
\text { design without a } \\
\text { placebo group } \\
\text {-Only included } \\
\text {-hospitalized } \\
\text { patients } \\
\text {-High number of } \\
\text { follow-up SARS- } \\
\text { CoV-2 (RT- } \\
\text {-PCR) tests were } \\
\text { not obtained } \\
\text { because of } \\
\text { the limited } \\
\text { resources } \\
\text { and variable } \\
\text { practices } \\
\text {-Premature } \\
\text { termination } \\
\text { could also have } \\
\text { led to an } \\
\text { •increased } \\
\text { data censoring } \\
\text { related to the } \\
\text { clinical } \\
\text {-outcome } \\
\text { - SoC group } \\
\text { included } \\
\text { patients treated } \\
\text { with other } \\
\text { antivirals }\end{array}$ & $\begin{array}{l}\text { HCQ and FVP combi- } \\
\text { nation therapy plus } \\
\text { SoC did not achieve } \\
\text { a higher efficacy than } \\
\text { SoC alone in patients } \\
\text { hospitalized with } \\
\text { moderate-to-severe } \\
\text { COVID-19. }[9(8,12) \text { vs. } \\
7(6,10) p=0.29]\end{array}$ \\
\hline $\begin{array}{l}\text { Chen et al. } \\
{[42]}\end{array}$ & $\begin{array}{l}\text { Favipiravir } \\
\text { versus Arbidol } \\
\text { for COVID-19: } \\
\text { A Randomized } \\
\text { Clinical Trial }\end{array}$ & $\begin{array}{l}\text {-Clinical recovery } \\
\text { rate at } 7 \text { days from } \\
\text { the beginning of } \\
\text { treatment } \\
\text {-Clinical recovery } \\
\text { was defined } \\
\text { as continuous } \\
(>72 \text { h) recovery }\end{array}$ & $\begin{array}{l}\text { FVP }(n=116) \\
\cdot C l i n i c a l \text { recovery rate } \\
\text { oD7: } 71(61.21 \%) \\
\text { ARB }(n=120) \\
\text {-Clinical recovery rate } \\
\text { oD7: } 62(51.67 \%)\end{array}$ & $\begin{array}{l}\text { FVP }(n=116) \\
\text {-Incidence of AOT or } \\
\text { NMV: } 21(18.1 \%) \\
\text {-Respiratory failure: } 1 \\
(0.9 \%) \\
\text { ARB ( } n=120) \\
\text {-Incidence of AOT or } \\
\text { NMV: } 27 \text { (22.5\%) } \\
\text {-Respiratory failure: } 4 \\
(3.3 \%)\end{array}$ & $\begin{array}{l}\text {-No clinically } \\
\text { proven effective } \\
\text { antiviral drug or } \\
\text { placebo as the } \\
\text { control arm } \\
\text {-Observation } \\
\text { time frame was } \\
\text { limited } \\
\text {-Did not require } \\
\text { positive nucleic } \\
\text { acid test in inclu- } \\
\text { sion criteria }\end{array}$ & $\begin{array}{l}\text { FVP did not improve } \\
\text { clinical recovery but } \\
\text { exhibited better } \\
\text { symptom relief than } \\
\text { ARB. [71 (61.21) vs. } 62 \\
\text { (51.67) } p=0.1396]\end{array}$ \\
\hline $\begin{array}{l}\text { Dabbous } \\
\text { et al. [42] }\end{array}$ & $\begin{array}{l}\text { Efficacy of favip- } \\
\text { iravir in COVID- } \\
19 \text { treatment: } \\
\text { a multi-center } \\
\text { randomized } \\
\text { study }\end{array}$ & $\begin{array}{l}\text {-Mortality rate } \\
\text { - Need for MV }\end{array}$ & $\begin{array}{l}\text { FVP }(n=44) \\
\text {-Mortality: } 1(2.3 \%) \\
\text {-Need for MV: } 0(0.0 \%) \\
\text { CQ }(n=48) \\
\text {-Mortality: } 2(4.2 \%) \\
\text {-Need for MV: } 4(8.3 \%)\end{array}$ & $\begin{array}{l}\mathrm{FVP}(\mathrm{n}=44) \\
\text {-Duration of hospital stay, } \\
\text { days: } 13.29 \pm 5.86 \\
\text { - } \mathrm{SpO}_{2}: \\
\text { o100-95\%: } 40(90.9 \%) \\
\text { o95-90\%: } 4(9.1 \%) \\
\text { o<90\%: } 0(0) \\
\mathrm{CQ}(\mathrm{n}=48) \\
\text {-Duration of hospital stay, } \\
\text { days: } 15.89 \pm 4.75 \\
\text { - } \mathrm{SpO}_{2}: \\
\text { o100-95\%: } 37(77.1 \%) \\
\text { o95-90\%: } 9(18.8 \%) \\
\text { o<90\%: } 2(4.2 \%)\end{array}$ & $\begin{array}{l}\text {-Not blinded } \\
\text {-No standard } \\
\text { care control } \\
\text {-Did not exam- } \\
\text { ine need for } \\
\text { ICU admission, } \\
\text { mortality or the } \\
\text { viremic response } \\
\text {-Included only } \\
\text { COVID-19 } \\
\text { patients who } \\
\text { were mildly or } \\
\text { moderately ill } \\
\text { and therefore } \\
\text { had a better } \\
\text { prognosis than } \\
\text { severely or criti- } \\
\text { cally ill patients }\end{array}$ & $\begin{array}{l}\text { FVP is a promising } \\
\text { drug for treatment of } \\
\text { COVID- } 19 \text { that might } \\
\text { decrease the hospital } \\
\text { stay and the need } \\
\text { for MV } \\
\text { Mortality rate: }[1 \text { ( } 2.3) \\
\text { vs. } 2 \text { (4.2) } p=1.00]\end{array}$ \\
\hline
\end{tabular}


Table 3 (continued)

\begin{tabular}{|c|c|c|c|c|c|c|}
\hline Author & Study name & Primary endpoint & Primary outcomes & Other outcomes & Limitations & Interpretation \\
\hline $\begin{array}{l}\text { Doi et al. } \\
{[48]}\end{array}$ & $\begin{array}{l}\text { A Prospective, } \\
\text { Randomized, } \\
\text { Open-Label Trial } \\
\text { of Early versus } \\
\text { Late } \\
\text { Favipiravir } \\
\text { Therapy in Hos- } \\
\text { pitalized Patients } \\
\text { with COVID-19 }\end{array}$ & $\begin{array}{l}\text {-Viral clearance by } \\
\text { day } 6\end{array}$ & $\begin{array}{l}\text { Early treatment FVP }(n=36) \\
\text {-SARS-CoV-2 clearance by day } 6: 66.7 \% \\
\text { Late treatment FVP }(n=33) \\
\text {-SARS-CoV-2 clearance by day } 6: 56.1 \%\end{array}$ & $\begin{array}{l}\text { Early treatment FVP } \\
(n=36) \\
\cdot \text { SARS-CoV-2 clearance by } \\
\text { day 10: } 86.1 \% \\
\cdot 50 \% \text { logarithmic reduc- } \\
\text { tion in the SARS-CoV-2 } \\
\text { viral load by day } 6: 94.4 \% \\
\text {-Median time until SARS- } \\
\text { CoV-2 clearance by local } \\
\text { RT-PCR: } 12.8 \\
\cdot \text { Disease progression or } \\
\text { death ( } n=44 \text { ): } 0.0 \\
\text { Late treatment FVP } \\
\text { ( } n=33 \text { ) } \\
\cdot \text { SARS-CoV-2 clearance by } \\
\text { day } 10: 83.1 \% \\
\cdot 50 \% \text { logarithmic reduc- } \\
\text { tion in the SARS-CoV-2 } \\
\text { viral load by day } 6: 78.8 \% \\
\cdot \text { Median time until SARS- } \\
\text { CoV-2 clearance by local } \\
\text { RT-PCR: } 17.8 \\
\cdot \text { Disease progression or } \\
\text { death ( } n=44 \text { ): } 0.0\end{array}$ & $\begin{array}{l}\text {-Small sample } \\
\text { size } \\
\text {-Unexpected } \\
\text { high frequency } \\
\text { of a negative RT- } \\
\text { PCR at the time } \\
\text { of enrollment } \\
\text { likely underpow- } \\
\text { ered the study } \\
\text {-Open-label } \\
\text { study design } \\
\text {-Staggered } \\
\text { treatment } \\
\text { design where all } \\
\text { patients eventu- } \\
\text { ally received FVP, } \\
\text { adopted due to } \\
\text { the unavailabil- } \\
\text { ity of placebo } \\
\text { at the time of } \\
\text { study concep- } \\
\text { tion, made it dif- } \\
\text { ficult to interpret } \\
\text { outcome differ- } \\
\text { ences beyond } \\
\text { the sixth day } \\
\text { •Only recruited } \\
\text { asymptomatic to } \\
\text { mildly sympto- } \\
\text { matic CovID-19 } \\
\text { patients } \\
\text {-Not known } \\
\text { whether early } \\
\text { treatment had } \\
\text { any impact on } \\
\text { replication-com- } \\
\text { petent viruses }\end{array}$ & $\begin{array}{l}\text { Administration of FVP } \\
\text { did not significantly } \\
\text { improve viral } \\
\text { clearance in the first } \\
6 \text { days, but there was } \\
\text { a trend toward earlier } \\
\text { viral clearance with } \\
\text { the agent. FVP was } \\
\text { associated with } \\
\text { numerical reduction in } \\
\text { time to defervescence, } \\
\text { and a significant } \\
\text { improvement in } \\
\text { fever was observed } \\
\text { the day after starting } \\
\text { therapy, compared } \\
\text { with findings with no } \\
\text { therapy. [66.7 (95\% } \\
\text { Cl, 51.4 to } 81.2 \text { ) vs. } \\
\text { 56.1 (95\% Cl, } 0.764 \\
\text { to } 2.623 \text { ) HR=1.416 } \\
\text { (0.764-2.623)] }\end{array}$ \\
\hline $\begin{array}{l}\text { Lou et al. } \\
\text { [30] }\end{array}$ & $\begin{array}{l}\text { Clinical } \\
\text { Outcomes } \\
\text { and Plasma } \\
\text { Concentrations } \\
\text { of Baloxavir } \\
\text { Marboxil and } \\
\text { Favipiravir } \\
\text { in COVID-19 } \\
\text { Patients: An } \\
\text { Exploratory } \\
\text { Randomized, } \\
\text { Controlled Trial }\end{array}$ & $\begin{array}{l}\text {-Viral negative rate } \\
\text { at } 14 \text { days } \\
\text { •Viral negative } \\
\text { was defined as } \\
\text { two consecutive } \\
\text { RT-PCR tests with } \\
\text { undetectable viral } \\
\text { RNA } \\
\text {-Time from } \\
\text { randomization to } \\
\text { clinical improve- } \\
\text { ment } \\
\text { •Improvement was } \\
\text { defined as either } \\
\text { increase by two } \\
\text { points on NEWS2 } \\
\text { or discharge from } \\
\text { the hospital }\end{array}$ & $\begin{array}{l}\text { Total }(n=29) \\
\text { •Viral negative, } n(\%) \\
\text { oD7: } 15(51.7 \%) \\
\text { oD14: } 24(82.8 \%) \\
\text { B/M }(n=10) \\
\text {-Viral negative, } n(\%) \\
\text { oD7: } 6(60.0 \%) \\
\text { oD14: } 7(70.0 \%) \\
\text { FVP ( } n=9) \\
\text { •Viral negative, n (\%) } \\
\text { oD7: } 4(44.4 \%) \\
\text { oD14: } 7(77.8 \%) \\
\text { Control }(n=10) \\
\text { •Viral negative, } n(\%) \\
\text { oD7: } 5(50.0 \%) \\
\text { oD14: } 10(100.0 \%)\end{array}$ & $\begin{array}{l}\text { Total }(n=29) \\
\cdot \text { Incidence of MV: } 1 \text { (3\%) } \\
\text { B/M ( } n=10) \\
\text { •Incidence of MV: } 0 \\
\text { FVP ( } n=9) \\
\cdot \text { Incidence of MV: } 0 \\
\text { Control }(n=10) \\
\cdot \text { Incidence of MV: } 1 \text { (10) }\end{array}$ & $\begin{array}{l}\text {-Small sample } \\
\text { size } \\
\text {-Subjects were } \\
\text { all under treat- } \\
\text { ment with other } \\
\text { medication } \\
\text {-The poor cor- } \\
\text { relation could } \\
\text { be due to the } \\
\text { delay between } \\
\text { infection and } \\
\text { treatment initia- } \\
\text { tion } \\
\text {-Patients in FVP } \\
\text { group showed } \\
\text { oldest average } \\
\text { age and shortest } \\
\text { time from symp- } \\
\text { tom onset to } \\
\text { randomization, } \\
\text { even though, } \\
\text { the clinical } \\
\text { performance of } \\
\text { FVP group was } \\
\text { not inferior to } \\
\text { the other two } \\
\text { groups } \\
\text { •Not blinded }\end{array}$ & $\begin{array}{l}\text { No extra benefit to } \\
\text { COVID-19 treatment } \\
\text { was observed when } \\
\text { adding B/M or FVP to } \\
\text { standard care } \\
\text { Viral negative rate at } \\
14 \text { days: [7 (70) vs. } 7 \\
\text { (77) vs. } 10 \text { (100)] } \\
\text { Time from randomi- } \\
\text { zation to clinical } \\
\text { improvement: [14 } \\
(6-49) \text { vs. } 14 \text { (6-38) vs. } \\
15 \text { (6-24)] }\end{array}$ \\
\hline
\end{tabular}


Table 3 (continued)

\begin{tabular}{|c|c|c|c|c|c|c|}
\hline Author & Study name & Primary endpoint & Primary outcomes & Other outcomes & Limitations & Interpretation \\
\hline $\begin{array}{l}\text { Shinkai } \\
\text { et al. [52] }\end{array}$ & $\begin{array}{l}\text { Efficacy and } \\
\text { Safety of Favip- } \\
\text { iravir in Moder- } \\
\text { ate COVID- } \\
19 \text { Pneumonia } \\
\text { Patients without } \\
\text { Oxygen Therapy: } \\
\text { A Randomized, } \\
\text { Phase III Clinical } \\
\text { Trial }\end{array}$ & $\begin{array}{l}\text {-Composite } \\
\text { outcome defined } \\
\text { as the time to } \\
\text { •improvement in } \\
\text { temperature, } \mathrm{SpO}_{2} \text {, } \\
\text { and findings on } \\
\text { chest imaging, and } \\
\text { recovery to SARS- } \\
\text { CoV-2-negative }\end{array}$ & $\begin{array}{l}\text { FVP }(n=107) \\
\text {-Number of patients who improved: } 81 \\
\text {-Median time to improvement: } 11.9 \\
\text { Placebo ( } n=49) \\
\text {-Number of patients who improved: } 28 \\
\text {-Median time to improvement: } 14.7\end{array}$ & 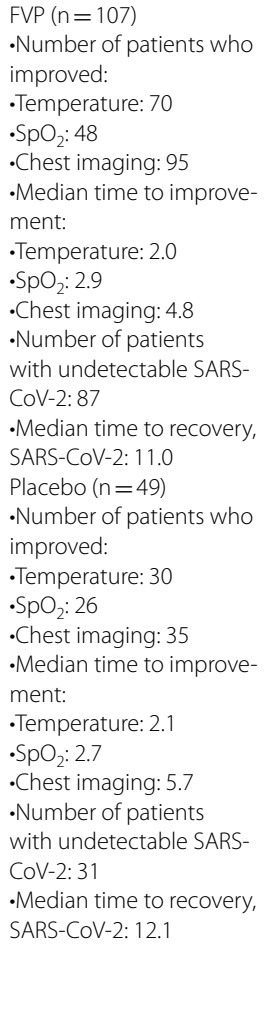 & $\begin{array}{l}\text {-Single-blind } \\
\text { design } \\
\text { •Virological } \\
\text { •investigations } \\
\text { were measured } \\
\text { solely by } \\
\text {-nasopharyngeal } \\
\text { swabs, despite } \\
\text { targeting } \\
\text { coviD-19 } \\
\text { patients with } \\
\text { pneumonia } \\
\text { •Difficulty in } \\
\text { recruiting only } \\
\text { suitable patients } \\
\text { of early-onset } \\
\text { for evaluating } \\
\text { antiviral drug } \\
\text { efficacy } \\
\text { •Only COVID-19 } \\
\text { patients with } \\
\text { moderate pneu- } \\
\text { monia } \\
\text { •(SpO } 2 \text { } 294 \%) \\
\text { •Primary } \\
\text { endpoint based } \\
\text { on CovID-19 } \\
\text { patient dis- } \\
\text { charge criterion } \\
\text { at that time } \\
\text { and cannot be } \\
\text { directly } \\
\text { •applied to the } \\
\text { current criterion }\end{array}$ & $\begin{array}{l}\text { FVP may be one of } \\
\text { options for moderate } \\
\text { COVID-19 pneumonia } \\
\text { treatment. However, } \\
\text { the risk of adverse } \\
\text { events, including } \\
\text { hyperuricemia, } \\
\text { should be carefully } \\
\text { considered. (11.9 vs. } \\
14.7 p=0.0136)\end{array}$ \\
\hline $\begin{array}{l}\text { Solaym- } \\
\text { ani- } \\
\text { Dodaran } \\
\text { et al. [44] }\end{array}$ & $\begin{array}{l}\text { Safety and effi- } \\
\text { cacy of Favipira- } \\
\text { vir in moderate } \\
\text { to severe } \\
\text { SARS-CoV-2 } \\
\text { pneumonia }\end{array}$ & $\begin{array}{l}\text {-Number of } \\
\text { admissions to the } \\
\text { intensive } \\
\text {-care unit }\end{array}$ & $\begin{array}{l}\text { FVP ( } n=190) \\
\cdot \mid C U \text { admission: } 31 \text { (16.3\%) } \\
\text { LPV/r }(n=183) \\
\cdot \mid C U \text { admission: } 25 \text { (13.7\%) }\end{array}$ & $\begin{array}{l}\text { FVP }(n=190) \\
\cdot \text { In-hospital mortality: } 26 \\
(13.7 \%) \\
\text {-Intubation: } 27(14.2 \%) \\
\text {-Length of hospital stay, } \\
\text { days }(n=153): 7(4,9) \\
\text {-Survival time till clinical } \\
\text { recovery, days ( } n=185) \text { : } \\
6(4,10) \\
\text { LPV/r }(n=183) \\
\text {-In-hospital mortality: } 21 \\
(11.5 \%) \\
\text {-Intubation: } 17(9.3 \%) \\
\text {-Length of hospital stay, } \\
\text { days }(n=150): 6(4,10) \\
\text {-Survival time till clinical } \\
\text { recovery, days }(n=182) \text { : } \\
6(4,10)\end{array}$ & $\begin{array}{l}\text {-Not blinded } \\
\text {-No control } \\
\text { group without } \\
\text { antivirals }\end{array}$ & $\begin{array}{l}\text { No clinical } \\
\text { benefit from a treat- } \\
\text { ment regimen based } \\
\text { on FVP in moderate to } \\
\text { severe cases of SARS- } \\
\text { CoV-2 over a treat- } \\
\text { ment regimen based } \\
\text { on LPV/r. [31 (16.3) vs. } \\
25 \text { (13.7) p=0.47] }\end{array}$ \\
\hline
\end{tabular}


Table 3 (continued)

\begin{tabular}{|c|c|c|c|c|c|c|}
\hline Author & Study name & Primary endpoint & Primary outcomes & Other outcomes & Limitations & Interpretation \\
\hline $\begin{array}{l}\text { Udwadia } \\
\text { et al. [35] }\end{array}$ & $\begin{array}{l}\text { Efficacy and } \\
\text { safety of favi- } \\
\text { piravir, an oral } \\
\text { RNA-dependent } \\
\text { RNA polymerase } \\
\text { inhibitor, in mild- } \\
\text { to-moderate } \\
\text { COVID-19: A } \\
\text { randomized, } \\
\text { comparative, } \\
\text { open-label, mul- } \\
\text { ticenter, phase } 3 \\
\text { clinical trial }\end{array}$ & $\begin{array}{l}\text {-Time from rand- } \\
\text { omization to the } \\
\text { cessation of oral } \\
\text { shedding of the } \\
\text { SARS-Cov-2 virus } \\
\text {-28 days maximum } \\
\text {-Defined as a } \\
\text { negative RT-PCR } \\
\text { result for both } \\
\text { oropharyngeal } \\
\text { and nasopharyn- } \\
\text { geal swabs }\end{array}$ & $\begin{array}{l}\text { FVP }(n=72) \\
\text {-Time to cessation of SARS-CoV-2 oral shed- } \\
\text { ding: } \\
\text { oNumber of events: } 70 \text { ( } 97.2 \%) \\
\text { oTime to event, median days: } 5.0 \\
\text { Control ( } n=75) \\
\text {-Time to cessation of SARS-CoV-2 oral shed- } \\
\text { ding: } \\
\text { oNumber of events: } 68 \text { ( } 90.7 \%) \\
\text { oTime to event, median days: } 7.0\end{array}$ & $\begin{array}{l}\text { FVP ( } n=72 \text { ) } \\
\text {-Time to clinical cure: } \\
\text { oNumber of events: } \\
51 / 53(96.2 \%) \\
\text { oTime to event, median } \\
\text { days: } 3.0 \\
\text {-Time to hospital } \\
\text { discharge: } \\
\text { oNumber of events: } \\
\text { 70/72 (97.2\%) } \\
\text { oTime to event, median } \\
\text { days: } 9.0 \\
\text { Control ( } n=75) \\
\text {-Time to clinical cure: } \\
\text { oNumber of events: } \\
\text { 46/49 ( } 93.9 \%) \\
\text { oTime to event, median } \\
\text { days: } 5.0 \\
\text {-Time to hospital } \\
\text { discharge: } \\
\text { oNumber of events: } \\
68 / 75 \text { ( } 90.7 \% \text { ) } \\
\text { oTime to event, median } \\
\text { days: } 10.0\end{array}$ & $\begin{array}{l}\text {-Primary } \\
\text { endpoint was } \\
\text { confounded } \\
\text { by interpreta- } \\
\text { tion issues with } \\
\text { RT-PCR positivity } \\
\text { and its lack of } \\
\text { correlation with } \\
\text { clinical cure } \\
\text {-Impact of } \\
\text { RT-PCR assay } \\
\text { variables such as } \\
\text { cycle time was } \\
\text { not evaluated } \\
\text { •Hazard ratios } \\
\text { observed much } \\
\text { smaller than pre- } \\
\text { viously reported } \\
\text {-Open-label } \\
\text { design }\end{array}$ & $\begin{array}{l}\text { Despite failure to } \\
\text { achieve statistical } \\
\text { significance on the pri- } \\
\text { mary endpoint of time } \\
\text { to RT-PCR negativity, } \\
\text { early administration of } \\
\text { oral FVP may reduce } \\
\text { the duration of clinical } \\
\text { signs and symptoms } \\
\text { in patients with mild- } \\
\text { to-moderate COVID- } \\
\text { 19, as demonstrated } \\
\text { by the significantly } \\
\text { decreased time to } \\
\text { clinical cure. [5 (95\% } \\
\text { Cl: 4-7) vs. } 7 \text { (95\% Cl } \\
\text { 5-8) p=0.129] }\end{array}$ \\
\hline $\begin{array}{l}\text { Zhao et al. } \\
\text { [45] }\end{array}$ & $\begin{array}{l}\text { Favipiravir in } \\
\text { the treatment } \\
\text { of patients with } \\
\text { SARS-CoV-2 } \\
\text { RNA recurrent } \\
\text { positive after } \\
\text { discharge: A } \\
\text { multicenter, } \\
\text { open-label, rand- } \\
\text { omized trial }\end{array}$ & $\begin{array}{l}\text {-Time to achieve } \\
\text { consecutive } \\
\text { twice (intervals } \\
\text { of more than } \\
24 \text { h) negative } \\
\text { RT-PCR result for } \\
\text { SARS-CoV-2 RNA } \\
\text { in nasopharyngeal } \\
\text { swab and sputum } \\
\text { sample }\end{array}$ & $\begin{array}{l}\text { FVP }(n=36) \\
\text {-SPD (SARS-CoV-2 RNA positive duration) } \\
\text { (days): } 28.3 \pm 16.6 \\
\text {-Proportion of RNA PCR turning negative: } \\
80.6 \%(29 / 36) \\
\text { Control }(n=19) \\
\text {-SPD (SARS-CoV-2 RNA positive duration) } \\
\text { (days): } 27.8 \pm 11.3 \\
\text {-Proportion of RNA PCR turning negative: } \\
52.6 \%(10 / 19)\end{array}$ & 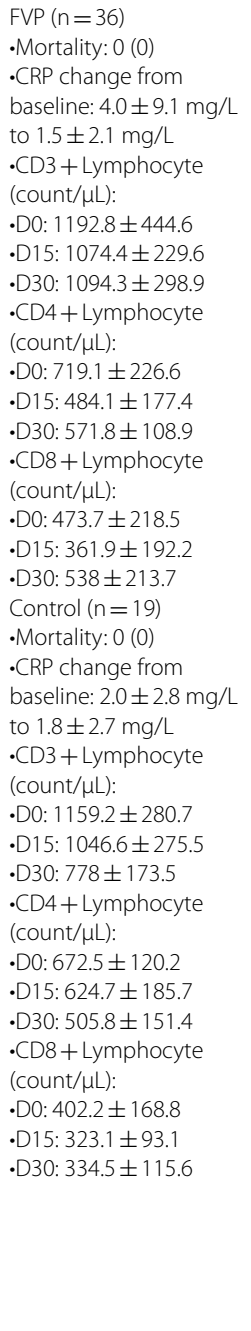 & $\begin{array}{l}\text {-Small sample } \\
\text { size } \\
\text {-Trial was not } \\
\text { blinded } \\
\text { •Followed up } \\
\text { all the patients } \\
\text { for only } 30 \text { days, } \\
\text { and it is not } \\
\text { clear whether } \\
\text { these patients } \\
\text { will return to } \\
\text { positive again } \\
\text {-Not been able } \\
\text { to obtain the } \\
\text { Ct value of } \\
\text { the dynamic } \\
\text { changes of } \\
\text { SARS-CoV-2 RNA } \\
\text { in patients } \\
\text { •Presence of few } \\
\text { symptomatic } \\
\text { patients in this } \\
\text { study, and only } \\
\text { mild symptoms, } \\
\text { prevents from } \\
\text { demonstrating } \\
\text { a clear clinical } \\
\text { benefit of FVP } \\
\text { •Hospital admis- } \\
\text { sion is manda- } \\
\text { tory in PCR } \\
\text { positive patients } \\
\text { in China, and } \\
\text { discharge is not } \\
\text { allowed mean- } \\
\text { while PCR is still } \\
\text { positive, but } \\
\text { these measures } \\
\text { are not followed } \\
\text { worldwide, so } \\
\text { the benefits of } \\
\text { treatment may } \\
\text { not be wide- } \\
\text { spread in other } \\
\text { settings }\end{array}$ & $\begin{array}{l}\text { FVP was safe and } \\
\text { superior to control in } \\
\text { shortening the dura- } \\
\text { tion of viral shedding } \\
\text { in SARS-CoV-2 RNA } \\
\text { recurrent positive after } \\
\text { discharge. [ } 27.8 \mathrm{vs} \text {. } \\
28.3 \mathrm{HR}=2.1(95 \% \mathrm{Cl} \\
\begin{array}{l}1.1-4.0) \mathrm{p}=0.038]\end{array}\end{array}$ \\
\hline
\end{tabular}


Table 3 (continued)

\begin{tabular}{|c|c|c|c|c|c|c|}
\hline Author & Study name & Primary endpoint & Primary outcomes & Other outcomes & Limitations & Interpretation \\
\hline $\begin{array}{l}\text { Ader et al. } \\
\text { [37] }\end{array}$ & $\begin{array}{l}\text { An open-label } \\
\text { randomized, } \\
\text { controlled trial } \\
\text { of the effect of } \\
\text { lopinavir/ritona- } \\
\text { vir, lopinavir/ } \\
\text { ritonavir plus } \\
\text { IFN- } \beta-1 \text { a and } \\
\text { hydroxychlo- } \\
\text { roquine in } \\
\text { hospitalized } \\
\text { patients with } \\
\text { COVID-19 }\end{array}$ & $\begin{array}{l}\text {-Clinical status at } \\
\text { day } 15, \text { measured } \\
\text { by the WHO } \\
\text { 7-point ordinal } \\
\text { scale } \\
\text {-7-point ordinal } \\
\text { scale: } \\
\text { o1. Not hospital- } \\
\text { ized/no } \\
\text { olimitations on } \\
\text { activities } \\
\text { o2. Not hospital- } \\
\text { ized, limitation } \\
\text { oon activities } \\
\text { o3. Hospitalized, } \\
\text { not requiring } \\
\text { osupplemental } \\
\text { oxygen } \\
\text { o4. Hospitalized, } \\
\text { requiring } \\
\text { osupplemental } \\
\text { oxygen } \\
\text { o5. Hospitalized, } \\
\text { on non-invasive } \\
\text { ventilation or } \\
\text { high flow oxygen } \\
\text { device } \\
\text { o6. Hospitalized, } \\
\text { on IMV or ECMO } \\
\text { o7. Death }\end{array}$ & 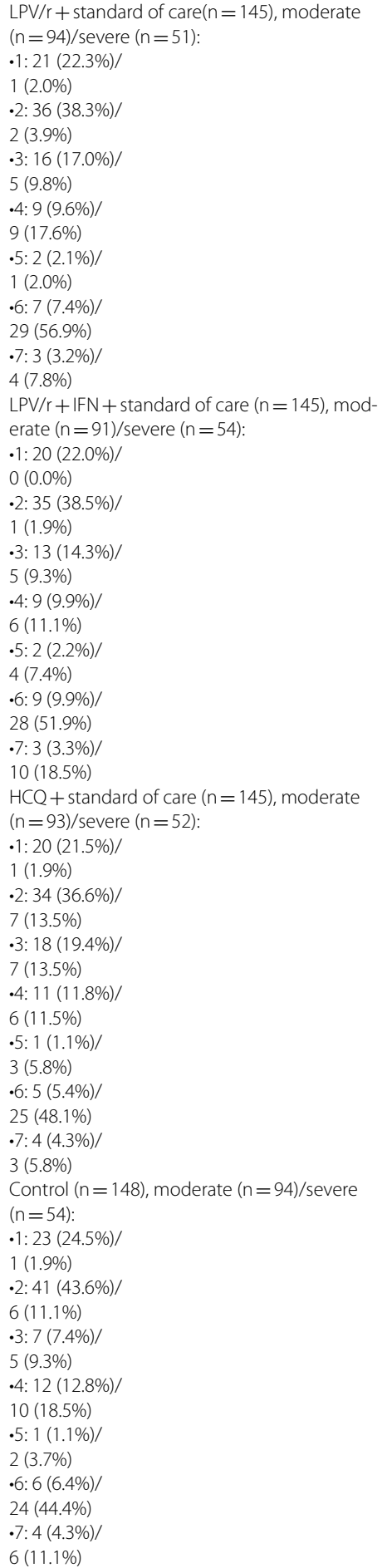 & 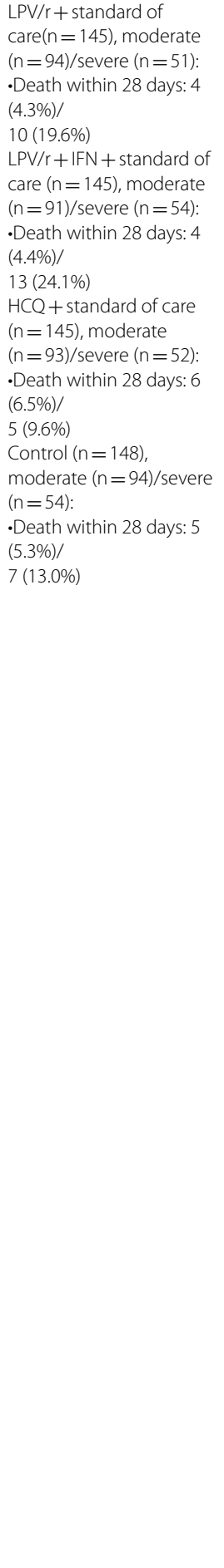 & $\begin{array}{l}\text {-Open-labelled } \\
\text { design } \\
\text { •Did not target } \\
\text { patients at the } \\
\text { early phase of } \\
\text { the disease } \\
\text {-Did not include } \\
\text { arms testing } \\
\text { anti-inflamma- } \\
\text { tory agents that } \\
\text { could be used } \\
\text { as part of the } \\
\text { standard of care } \\
\text { arm } \\
\text {-Standard of care } \\
\text { - underwent sub- } \\
\text { stantial changes } \\
\text { over time }\end{array}$ & $\begin{array}{l}\text { In patients admitted } \\
\text { to hospital with } \\
\text { COVID-19, LVP/r, LVP/r } \\
\text { plus IFN- } \beta-1 \text { a and HCQ } \\
\text { were not associated } \\
\text { with clinical improve- } \\
\text { ment at day } 15 \text { and } \\
\text { day } 29 \text {, nor reduction } \\
\text { in viral shedding. [aOR } \\
0.83(95 \% \mathrm{Cl} 0.55-1.26 \\
p=0.39) \text { vs. aOR } 0.69 \\
(95 \% \mathrm{Cl} 0.45-1.04 \\
p=0.08) \text { vs. aOR } 0.93 \\
(95 \% \mathrm{Cl} 0.62-1.41 \\
p=0.75)]\end{array}$ \\
\hline
\end{tabular}


Table 3 (continued)

\begin{tabular}{|c|c|c|c|c|c|c|}
\hline Author & Study name & Primary endpoint & Primary outcomes & Other outcomes & Limitations & Interpretation \\
\hline $\begin{array}{l}\text { Alavi } \\
\text { Darazam } \\
\text { et al. [47] }\end{array}$ & $\begin{array}{l}\text { Umifenovir in } \\
\text { hospitalized } \\
\text { moderate to } \\
\text { severe COVID-19 } \\
\text { patients: A } \\
\text { randomized } \\
\text { clinical trial }\end{array}$ & $\begin{array}{l}\text {-Time clinical } \\
\text { improvement } \\
\text { evaluated based } \\
\text { on improvement } \\
\text { of two points of } \\
\text { the seven-cate- } \\
\text { gory ordinal scale } \\
\text { (recommended by } \\
\text { the World Health } \\
\text { Organization) or } \\
\text { discharge from the } \\
\text { hospital, } \\
\text {-whichever came } \\
\text { first }\end{array}$ & $\begin{array}{l}\mathrm{LPV} / \mathrm{r}+\mathrm{HCQ}+\mathrm{IFN}-\beta-1 \mathrm{a}+\mathrm{ARB}(\mathrm{n}=51) \\
\text {-Time to clinical } \\
\text {-improvement: } 9(5-11) \\
\text { Control }(\mathrm{n}=50) \\
\text {-Time to clinical } \\
\text {-improvement, median: } 7(4-10)\end{array}$ & $\begin{array}{l}\text { LPV/r + HCQ + IFN- } \\
\beta-1 a+A R B(n=51) \\
\text {-Mortality at D } \\
\text {-21: } 17(33.3 \%) \\
\text {-ICU adm: } 51(100.0 \%) \\
\text {-IMV: } 17(33.3 \%) \\
\text { Control }(n=50) \\
\text {-Mortality at D } \\
\text {-21: } 19(38.0 \%) \\
\text {-ICU adm: } 50(100.0 \%) \\
\text {-IMV: } 14(28.0 \%)\end{array}$ & $\begin{array}{l}\text {-Not blinded } \\
\text {-38 patients } \\
\text { unable to com- } \\
\text { plete treatment } \\
\text { course of admin- } \\
\text { istration because } \\
\text { of liver enzyme } \\
\text { elevation } \\
\text {-The trial was } \\
\text {-conducted on } \\
\text { hospitalized } \\
\text { patients with } \\
\text { moderate- } \\
\text { severe CovID-19 } \\
\text { and the } \\
\text { effectiveness of } \\
\text { umifenovir in } \\
\text { patients with } \\
\text { mild Covid-19 } \\
\text { not } \\
\text { •evaluated }\end{array}$ & $\begin{array}{l}\text { Additive ARB was not } \\
\text { effective in } \\
\text { shortening the dura- } \\
\text { tion of SARS-CoV- } 2 \text { in } \\
\text { severe patients and } \\
\text { improving } \\
\text { the prognosis in non- } \\
\text { ICU patients. [ } 9(5-11) \\
\text { vs. } 7(4-10) p=0.22 \text { ] }\end{array}$ \\
\hline $\begin{array}{l}\text { Arabi et al. } \\
{[38]}\end{array}$ & $\begin{array}{l}\text { Lopinavir-riton- } \\
\text { avir and hydroxy- } \\
\text { chloroquine } \\
\text { for critically ill } \\
\text { patients with } \\
\text { COVID-19: } \\
\text { REMAP-CAP } \\
\text { randomized } \\
\text { controlled trial }\end{array}$ & $\begin{array}{l}\text {-Ordinal scale of } \\
\text { organ support-free } \\
\text { days }\end{array}$ & $\begin{array}{l}\text { LPV/r }(n=225) \\
\cdot \text { Organ support-free days: } 4(-1,15) \\
\text { HCQ }(n=50) \\
\text {-Organ support-free days: } 0(-1,9) \\
\text { Combination therapy }(n=27) \\
\text {-Organ support-free days: }-1(-1,7) \\
\text { Control }(n=362) \\
\text {-Organ support-free days: } 6(-1,16)\end{array}$ & 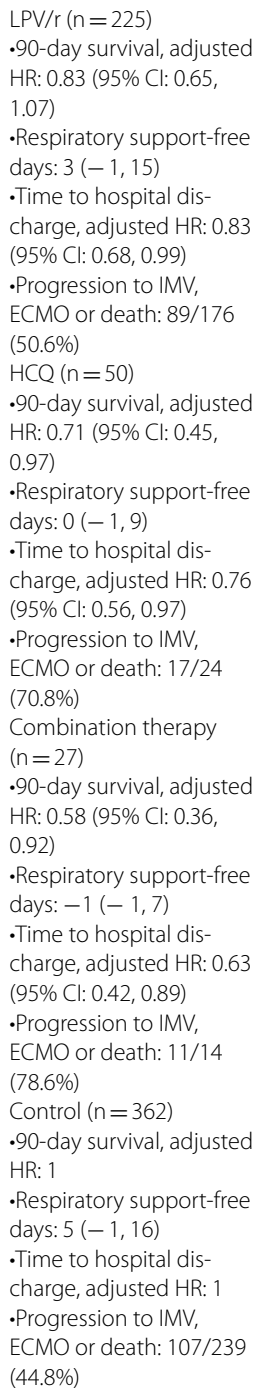 & $\begin{array}{l}\text {-Data on the } \\
\text { bioavailability } \\
\text { of dissolved or } \\
\text { crushed } \\
\text {-LPV/r tablets } \\
\text { in critically ill } \\
\text { patients are } \\
\text { limited } \\
\text { - Open-label } \\
\text { design }\end{array}$ & $\begin{array}{l}\text { Among critically ill } \\
\text { patients with } \\
\text { COVID-19, treatment } \\
\text { with LPV/r, HCQ, or } \\
\text { combination therapy } \\
\text { resulted in worse out- } \\
\text { comes compared to } \\
\text { no antiviral therapy. [4 } \\
(-1,15) \text { vs. } 0(-1,9) \text { vs. } \\
-1(-1,7) \text { vs. } 6(-1,16)]\end{array}$ \\
\hline
\end{tabular}


Table 3 (continued)

\begin{tabular}{|c|c|c|c|c|c|c|}
\hline Author & Study name & Primary endpoint & Primary outcomes & Other outcomes & Limitations & Interpretation \\
\hline $\begin{array}{l}\text { Cao et al. } \\
{[24]}\end{array}$ & $\begin{array}{l}\text { A Trial of Lopina- } \\
\text { vir-Ritonavir in } \\
\text { Adults Hospital- } \\
\text { ized with Severe } \\
\text { Covid-19 }\end{array}$ & $\begin{array}{l}\text {-Time to clinical } \\
\text { improvement, } \\
\text { defined as the } \\
\text { time from rand- } \\
\text { omization to either } \\
\text { an improvement } \\
\text { of two points on } \\
\text { a seven-category } \\
\text { ordinal scale or } \\
\text { discharge from the } \\
\text { hospital, which- } \\
\text { ever came first }\end{array}$ & $\begin{array}{l}\text { LPV/r }(n=99) \\
\text {-Time to clinical improvement, days: } 16.0 \text { (13.0, } \\
\text { 17.0) } \\
\text { Control }(n=100) \\
\text {-Time to clinical improvement, days: } 16.0 \text { (15.0, } \\
\text { 18.0) }\end{array}$ & 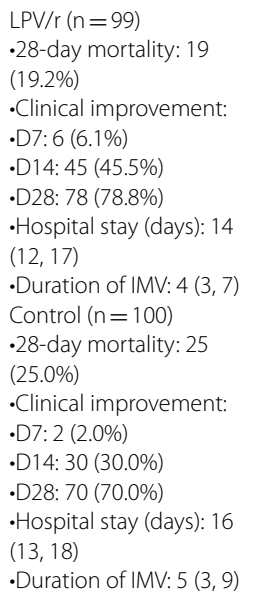 & $\begin{array}{l}\text {-Not blinded } \\
\text {-Characteristics } \\
\text { of the patients } \\
\text { at baseline were } \\
\text { generally bal- } \\
\text { anced across the } \\
\text { two groups, but } \\
\text { the somewhat } \\
\text { higher throat } \\
\text { viral loads in } \\
\text { the LPV/r group } \\
\text { raise the pos- } \\
\text { sibility that this } \\
\text { group had more } \\
\text { viral replication } \\
\text {-Do not have } \\
\text { data on the LPV } \\
\text { exposure levels } \\
\text { in patients }\end{array}$ & $\begin{array}{l}\text { In hospitalized } \\
\text { patients with severe } \\
\text { COVID-19, LPV/r } \\
\text { showed no benefit } \\
\text { compared to standard } \\
\text { care. [16 vs. } 16 \\
\text { HR }=1.31,95 \% \mathrm{Cl} \\
(0.95-1.85), p=0.09]\end{array}$ \\
\hline $\begin{array}{l}\text { Li et al. } \\
\text { [29] }\end{array}$ & $\begin{array}{l}\text { Efficacy and } \\
\text { safety of lopina- } \\
\text { vir/ritonavir or } \\
\text { arbidol in adult } \\
\text { patients with } \\
\text { mild/moder- } \\
\text { ateCOVID-19: } \\
\text { an exploratory } \\
\text { randomized } \\
\text { controlled trial }\end{array}$ & $\begin{array}{l}\text {-Rate of positive- } \\
\text { to-negative } \\
\text { conversion of } \\
\text { SARS-CoV-2 } \\
\text { nucleic acid }\end{array}$ & $\begin{array}{l}\text { LPV/r }(n=34) \\
\text {-Positive-to-negative conversion of SARS-CoV-2 } \\
\text { nucleic acid by pharyngeal swab } \\
\text {-D7: } 12(35.3 \%) \\
\text { ARB }(n=35) \\
\text {-Positive-to-negative conversion of SARS-CoV-2 } \\
\text { nucleic acid by pharyngeal swab } \\
\text {-D7: } 13(37.1 \%) \\
\text { Control }(n=17) \\
\text {-Positive-to-negative conversion of SARS-CoV-2 } \\
\text { nucleic acid by pharyngeal swab } \\
\text {-D7: } 7(41.2 \%)\end{array}$ & $\begin{array}{l}\text { LPV/r ( } n=34 \text { ) } \\
\text {-Positive-to-negative } \\
\text { conversion of SARS- } \\
\text { CoV-2 nucleic acid by } \\
\text { pharyngeal swab } \\
\text {-D14: } 29 \text { (85.3\%) } \\
\text {-Time of positive-to- } \\
\text { negative conversion } \\
\text { of SARS-CoV-2 nucleic } \\
\text { acid in pharyngeal swab } \\
\text { (days): } 9.0 \pm 5.0 \\
\text {-Conversion rate from } \\
\text { moderate to severe/ } \\
\text { critical clinical status: } 8 \\
\text { (23.5\%) } \\
\text { ARB ( } n=35 \text { ) } \\
\text {-Positive-to-negative } \\
\text { conversion of SARS- } \\
\text { CoV-2 nucleic acid by } \\
\text { pharyngeal swab } \\
\text {-D14: } 32 \text { (91.4\%) } \\
\text {-Time of positive-to- } \\
\text { negative conversion of } \\
\text { SARS-CoV-2 nucleic acid } \\
\text { in pharyngeal swab, days: } \\
\text { 9.1 } \pm 4.4 \\
\text {-Conversion rate from } \\
\text { moderate to severe/criti- } \\
\text { cal clinical status: } 3 \text { ( } 8.6 \% \text { ) } \\
\text { Control ( } n=17 \text { ) } \\
\text {-Positive-to-negative } \\
\text { conversion of SARS- } \\
\text { CoV-2 nucleic acid by } \\
\text { pharyngeal swab } \\
\text { oD14: } 13 \text { (76.5\%) } \\
\text {-Time of positive-to- } \\
\text { negative conversion of } \\
\text { SARS-CoV-2 nucleic acid } \\
\text { in pharyngeal swab, days: } \\
\text { 9.3 } \pm 5.2 \\
\text {-Conversion rate from } \\
\text { moderate to severe/ } \\
\text { critical clinical status: } 2 \\
\text { (11.8\%) }\end{array}$ & $\begin{array}{l}\text {-Small sample } \\
\text { size } \\
\text {-Did not include } \\
\text { severely or criti- } \\
\text { cally ill patients } \\
\text { or patients at } \\
\text { increased risk of } \\
\text { poor outcomes } \\
\text { with many } \\
\text { comorbidities } \\
\text { - Not completely } \\
\text { blinded }\end{array}$ & $\begin{array}{l}\text { LPV/r and ARB therapy } \\
\text { show little benefit } \\
\text { for improving clinical } \\
\text { outcome in hospital- } \\
\text { ized patients with mild } \\
\text { to moderate COVID-19 } \\
\text { compared to support- } \\
\text { ive care. [35.3 vs. } 37.1 \\
\text { vs. } 41.2 p=0.966 \text { ] }\end{array}$ \\
\hline
\end{tabular}


Table 3 (continued)

\begin{tabular}{|c|c|c|c|c|c|c|}
\hline Author & Study name & Primary endpoint & Primary outcomes & Other outcomes & Limitations & Interpretation \\
\hline $\begin{array}{l}\text { Nojomi } \\
\text { et al. [31] }\end{array}$ & $\begin{array}{l}\text { Effect of Arbidol } \\
\text { (Umifenovir) on } \\
\text { COVID-19: } \\
\text { a randomized } \\
\text { controlled trial }\end{array}$ & $\begin{array}{l}\text {-Duration of hospi- } \\
\text { talization } \\
\text {-Time to clinical } \\
\text { improvement }\end{array}$ & $\begin{array}{l}\text { LPV/r }(n=50) \\
\text {-Duration of hospitalization, days: } 9.6 \pm 5.2 \\
\text {-Time to clinical improvement: } 3.1 \pm 1.4 \\
\text { ARB ( } n=50) \\
\text {-Duration of hospitalization, days: } 7.2 \pm 4.7 \\
\text {-Time to clinical improvement: } 2.7 \pm 1.1\end{array}$ & $\begin{array}{l}\text { LPV/r }(n=50) \\
\cdot 30-\text { day mortality: } 2 \\
(4.0 \%) \\
\cdot \text { IMV: } 2(4.0 \%) \\
\text { ARB ( } n=50) \\
\cdot 30-\text { day mortality: } 1 \\
(2.0 \%) \\
\cdot \text { IMV: } 3(6.0 \%)\end{array}$ & $\begin{array}{l}\text {-Not blinded } \\
\text {-Treatments } \\
\text { were given in } \\
\text { combination } \\
\text { with HCQ } \\
\text {-Small sample } \\
\text { sizes for disease } \\
\text { severity sub- } \\
\text { groups }\end{array}$ & $\begin{array}{l}\text { ARB significantly } \\
\text { shortens duration } \\
\text { of hospitalization } \\
\text { compared to LPV/r } \\
\text { in patients with } \\
\text { COVID-19 } \\
\text { Duration of hospi- } \\
\text { talization: ( } 7.2 \text { vs. } 9.6 \\
\text { p=0.02) } \\
\text { Time to clinical } \\
\text { improvement: ( } 2.7 \\
\text { vs. 3.1) }\end{array}$ \\
\hline $\begin{array}{l}\text { RECOVERY } \\
\text { collabora- } \\
\text { tive group } \\
{[26]}\end{array}$ & $\begin{array}{l}\text { Lopinavir-rito- } \\
\text { navir in patients } \\
\text { admitted to } \\
\text { hospital with } \\
\text { COVID-19 } \\
\text { (RECOVERY): } \\
\text { a randomised, } \\
\text { controlled, open- } \\
\text { label, platform } \\
\text { trial }\end{array}$ & $\begin{array}{l}\text { •28-day all-cause } \\
\text { mortality }\end{array}$ & $\begin{array}{l}\text { LPV/r }(n=1616) \\
\text {-28-day mortality: } 374(23 \%) \\
\text { Standard care }(n=3424) \\
\text { •28-day mortality: } 767(22 \%)\end{array}$ & $\begin{array}{l}\text { LPV/r }(n=1616) \\
\text {-Discharged from } \\
\text { hospital within } 28 \text { days: } \\
1113(69 \%) \\
\text {-IMV: } 152 / 1556(10 \%) \\
\text {-Death: 350/1556 (22\%) } \\
\text { Standard care }(n=3424) \\
\text {-Discharged from } \\
\text { hospital within } 28 \text { days: } \\
2382(70 \%) \\
\text {-IMV: } 279 / 3280(9 \%) \\
\text {-Death: } 712 / 3280(22 \%)\end{array}$ & $\begin{array}{l}\text {-Not blinded } \\
\text {-Did not col- } \\
\text { lect detailed } \\
\text { information on } \\
\text { non-serious } \\
\text { adverse reac- } \\
\text { tions or reasons } \\
\text { for stopping } \\
\text { treatment } \\
\text {-Did not collect } \\
\text { information } \\
\text { on physiologi- } \\
\text { cal, laboratory, } \\
\text { or virological } \\
\text { parameters } \\
\text {-Very few } \\
\text { intubated } \\
\text { patients with } \\
\text { coviD-19 were } \\
\text { enrolled in this } \\
\text { study as there } \\
\text { were difficulties } \\
\text { in administer- } \\
\text { ing treatment } \\
\text { to patients } \\
\text { who could not } \\
\text { swallow }\end{array}$ & $\begin{array}{l}\text { LPV/r was not associ- } \\
\text { ated with reduction in } \\
28 \text {-day mortality, dura- } \\
\text { tion of hospital stay, or } \\
\text { risk of progression to } \\
\text { IMV or death. [23 vs. } \\
22,95 \% \mathrm{Cl}(0.91-1.17) \\
\mathrm{p}=0.60]\end{array}$ \\
\hline $\begin{array}{l}\text { Reis et al. } \\
{[27]}\end{array}$ & $\begin{array}{l}\text { Effect of Early } \\
\text { Treatment With } \\
\text { Hydroxychlo- } \\
\text { roquine or } \\
\text { Lopinavir and } \\
\text { Ritonavir } \\
\text { on Risk of Hospi- } \\
\text { talization Among } \\
\text { Patients With } \\
\text { COVID-19 } \\
\text { The TOGETHER } \\
\text { Randomized } \\
\text { Clinical Trial }\end{array}$ & $\begin{array}{l}\cdot \text { COVID-19-asso- } \\
\text { ciated } \\
\text { •hospitalization } \\
\text { and death } 90 \text { days } \\
\text { after randomiza- } \\
\text { tion }\end{array}$ & $\begin{array}{l}\text { HCQ }(n=214) \\
\text {-COVID-19 hospitalization: } 8(3.7 \%) \\
\text {-Death: } 0(0.0 \%) \\
\text { LPV/r }(n=244) \\
\text {-COVID-19 hospitalization: } 14(5.7 \%) \\
\text {-Death: } 2(0.8 \%) \\
\text { Placebo }(n=227) \\
\text {-COVID-19 hospitalization: } 11(4.8 \%) \\
\text {-Death: } 1(0.4 \%)\end{array}$ & $\begin{array}{l}\text { HCQ }(n=214) \\
\text {-All-cause hospitalization: } \\
11(5.1 \%) \\
\text {-Time to viral clearance } \\
(n=185): 97(52.4 \%) \\
\text { LPV/r }(n=244) \\
\text {-All-cause hospitalization: } \\
16(6.6 \%) \\
\text {-Time to viral clearance } \\
\text { ( } n=201): 125(62.2 \%) \\
\text { Placebo ( } n=227) \\
\text {-All-cause hospitalization: } \\
12(5.3 \%) \\
\text {-Time to viral clearance } \\
\text { ( } n=195): 112 \text { (57.4\%) }\end{array}$ & $\begin{array}{l}\text {-Found a } \\
\text { low rate of } \\
\text { hospitalizations, } \\
\text { even though } \\
\text { the population } \\
\text { had risk factors } \\
\text { for developing } \\
\text { serious COVID- } \\
19 \text { and median } \\
\text { (range) age of } 53 \\
\text { (18-94) years }\end{array}$ & $\begin{array}{l}\text { No clinical benefit to } \\
\text { support the use of } \\
\text { either } \\
\text { HCQ or LPV/r in an } \\
\text { outpatient population } \\
\text { Hospitalization: [8 (3.7) } \\
\text { vs. } 14(5.7) \text { vs. } 11(4.8)] \\
\text { Death: [0 (0) vs. } 2(0.8) \\
\text { vs. } 1(0.4)]\end{array}$ \\
\hline
\end{tabular}


Table 3 (continued)

\begin{tabular}{|c|c|c|c|c|c|c|}
\hline Author & Study name & Primary endpoint & Primary outcomes & Other outcomes & Limitations & Interpretation \\
\hline $\begin{array}{l}\text { Barratt- } \\
\text { Due et al. } \\
{[39]}\end{array}$ & $\begin{array}{l}\text { Evaluation of } \\
\text { the Effects of } \\
\text { Remdesivir and } \\
\text { Hydroxychloro- } \\
\text { quine on Viral } \\
\text { Clearance in } \\
\text { COVID-19: A } \\
\text { Randomized Trial }\end{array}$ & $\begin{array}{l}\text {-All-cause, in- } \\
\text { •hospital mortality }\end{array}$ & 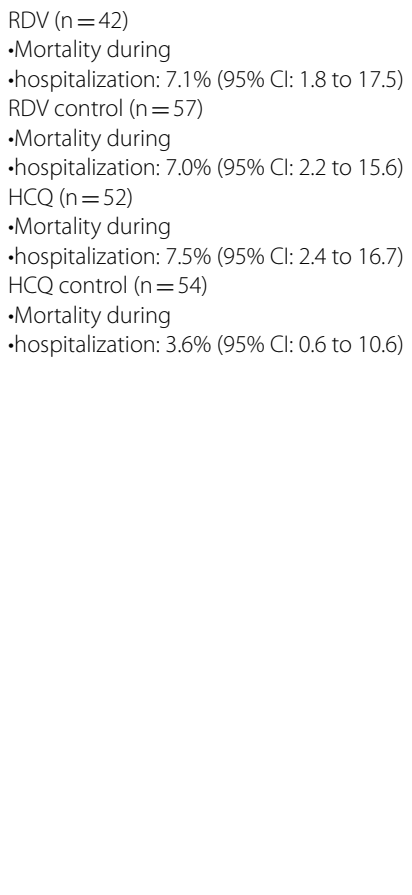 & 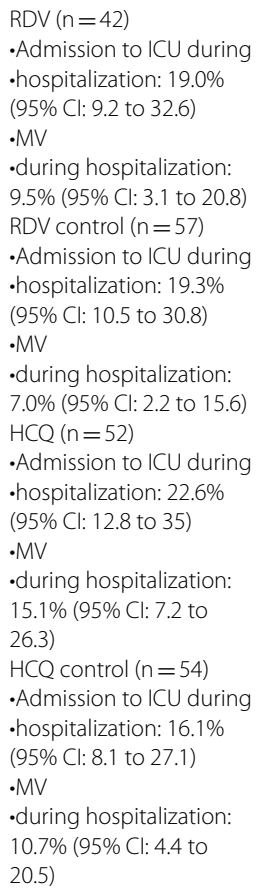 & $\begin{array}{l}\text {-Not blinded } \\
\text { - Relatively few } \\
\text {-patients were } \\
\text { included, and } \\
\text { Cls were wide } \\
\text { enough to } \\
\text { include moder- } \\
\text { ate effects } \\
\text { - Not all data } \\
\text { were available } \\
\text { from all patients } \\
\text { at all } \\
\text {-time points } \\
\text {-Most of the } \\
\text { patients did } \\
\text { not receive the } \\
\text { full treatment } \\
\text { length } \\
\text { •of the tested } \\
\text { medication } \\
\text { due to hospital } \\
\text { discharge }\end{array}$ & $\begin{array}{l}\text { Neither RDV nor } \\
\text { HCQ affected viral } \\
\text { clearance in hospital- } \\
\text { ized patients with } \\
\text { COVID-19 } \\
\text { [7.1 vs. } 7.0 \text { vs. } 7.5 \\
\text { vs. } 3.6]\end{array}$ \\
\hline $\begin{array}{l}\text { Beigel } \\
\text { et al. [23] }\end{array}$ & $\begin{array}{l}\text { Remdesivir for } \\
\text { the Treatment of } \\
\text { Covid-19_Final } \\
\text { Report }\end{array}$ & $\begin{array}{l}\text {-Time to recovery } \\
\text { - Defined by either } \\
\text { discharge from } \\
\text { the hospital or } \\
\text { hospitalization for } \\
\text { infection-control } \\
\text { purposes only }\end{array}$ & $\begin{array}{l}\text { RDV }(n=541) \\
\text {-Time to recovery: } 10(9,11) \\
\text { Control }(n=521) \\
\text {-Time to recovery: } 15(13,18)\end{array}$ & $\begin{array}{l}\text { RDV ( } n=541) \\
\text {-Recovery: } 399 \text { (73.8\%) } \\
\text {-29-day mortality: } 59 \\
(10.9 \%) \\
\text {-Time to clinical improve- } \\
\text { ment, one category on } \\
\text { ordinal scale, days } 7.0 \\
\text { (6.0, } 8.0) \\
\text {-Duration of initial hospi- } \\
\text { talization, days: } 12(6,28) \\
\text {-New use of MV or ECMO: } \\
52 / 402(12.9 \%) \\
\text { Control ( } n=521) \\
\text { - Recovery: } 352 \text { (67.6\%) } \\
\text {-29-day mortality: } 77 \\
\text { (14.8\%) } \\
\text {-Time to clinical improve- } \\
\text { ment, one category on } \\
\text { ordinal scale, days: } 9.0 \\
\text { (8.0, } 11.0) \\
\text {-Duration of initial hospi- } \\
\text { talization, days: } 17 \text { (8, 28) } \\
\text { - New use of MV or ECMO: } \\
82 / 364 \text { (22.5\%) }\end{array}$ & $\begin{array}{l}\text {-Training, site } \\
\text { initiation visits, } \\
\text { and monitor- } \\
\text { ing visits often } \\
\text { were performed } \\
\text { remotely due to } \\
\text { restricted travel } \\
\text { and hospital } \\
\text { restriction of } \\
\text { entrance of } \\
\text { nonessential } \\
\text { personnel } \\
\text {-Research staff } \\
\text { were often } \\
\text { assigned other } \\
\text { clinical duties } \\
\text { and staff ill- } \\
\text { nesses strained } \\
\text { research } \\
\text { resources } \\
\text { •Many sites } \\
\text { did not have } \\
\text { adequate sup- } \\
\text { plies of personal } \\
\text { protective } \\
\text { equipment and } \\
\text { trial-related } \\
\text { supplies, such as } \\
\text { swabs }\end{array}$ & $\begin{array}{l}\text { RDV shortens time } \\
\text { to recovery in hos- } \\
\text { pitalized COVID-19 } \\
\text { patients with evidence } \\
\text { of infection in the } \\
\text { lower respiratory tract } \\
{[10 \text { days vs. } 15 \text { days, }} \\
p<0.001]\end{array}$ \\
\hline
\end{tabular}


Table 3 (continued)

\begin{tabular}{|c|c|c|c|c|c|c|}
\hline Author & Study name & Primary endpoint & Primary outcomes & Other outcomes & Limitations & Interpretation \\
\hline $\begin{array}{l}\text { Goldman } \\
\text { et al. [53] }\end{array}$ & $\begin{array}{l}\text { Remdesivir for } \\
5 \text { or } 10 \text { Days in } \\
\text { Patients } \\
\text { with Severe } \\
\text { Covid-19 }\end{array}$ & $\begin{array}{l}\text {-Clinical status } \\
\text { assessed on D14 } \\
\text { on a 7-point } \\
\text { ordinal scale } \\
\text { o1. death } \\
\text { o2. hospitalized, } \\
\text { receiving IMV or } \\
\text { ECMO } \\
\text { o3. hospitalized, } \\
\text { receiving noninva- } \\
\text { sive ventilation or } \\
\text { high-flow oxygen } \\
\text { devices } \\
\text { o4. hospitalized, } \\
\text { requiring low-flow } \\
\text { supplemental } \\
\text { oxygen } \\
\text { o5. hospitalized, } \\
\text { not requiring sup- } \\
\text { plemental oxygen } \\
\text { but receiving } \\
\text { ongoing medica } \\
\text { care (related or } \\
\text { not related to } \\
\text { Covid-19); } \\
\text { o6. hospitalized, } \\
\text { requiring neither } \\
\text { supplemental } \\
\text { oxygen nor ongo- } \\
\text { ing medical care } \\
\text { (other than that } \\
\text { specified in the } \\
\text { protocol for RDV } \\
\text { administration) } \\
\text { o7. not hospital- } \\
\text { ized }\end{array}$ & $\begin{array}{l}\text { 5-day RDV }(\mathrm{n}=200) \\
\cdot \text { Clinical status at day } 14 \text { on the 7-point ordinal } \\
\text { scale: } \\
\cdot 1: 16(8.0 \%) \\
\cdot 2: 16(8.0 \%) \\
\cdot 3: 9(4.5 \%) \\
\cdot 4: 19(9.5 \%) \\
\cdot 5: 11(5.5 \%) \\
\cdot 6: 9(4.5 \%) \\
\cdot 7: 120(60.0 \%) \\
\text { 10-day RDV }(\mathrm{n}=197) \\
\cdot \text { Clinical status at day } 14 \text { on the 7-point ordinal } \\
\text { scale: } \\
\cdot 1: 21(10.5 \%) \\
\cdot 2: 33(16.5 \%) \\
\cdot 3: 10(5.0 \%) \\
\cdot 4: 14(7.0 \%) \\
\cdot 5: 13(6.5 \%) \\
\cdot 6: 3(1.5 \%) \\
\cdot 7: 103(51.5 \%)\end{array}$ & $\begin{array}{l}\text { 5-day RDV }(\mathrm{n}=200) \\
\text {-Time to clinical } \\
\text { improvement (median } \\
\text { day of 50\% cumulative } \\
\text { incidence): } 10 \\
\text {-Time to recovery } \\
\text { (median day of 50\% } \\
\text { cumulative incidence): } 10 \\
\text { 10-day RDV ( } \mathrm{n}=197 \text { ) } \\
\text {-Time to clinical } \\
\text { improvement (median } \\
\text { day of 50\% cumulative } \\
\text { incidence): } 11 \\
\text {-Time to recovery } \\
\text { (median day of 50\% } \\
\text { cumulative incidence): } 11\end{array}$ & $\begin{array}{l}\text {-Not blinded } \\
\text {-Did not have } \\
\text { SARS-CoV-2 } \\
\text { viral-load results } \\
\text { during and } \\
\text { after treatment, } \\
\text { owing to the } \\
\text { variability in } \\
\text { local access } \\
\text { to testing and } \\
\text { practices across } \\
\text { the global sites }\end{array}$ & $\begin{array}{l}\text { No significant dif- } \\
\text { ference was found } \\
\text { between a } 5 \text {-day } \\
\text { course and a } 10 \text {-day } \\
\text { course of RDV in } \\
\text { patients with severe } \\
\text { Covid-19 not requir- } \\
\text { ing MV } \\
\text { [65.2 vs. 57.1, 95\% Cl } \\
(1.16-1.90) p=0.002]\end{array}$ \\
\hline $\begin{array}{l}\text { Mahajan } \\
\text { et al. [51] }\end{array}$ & $\begin{array}{l}\text { Clinical out- } \\
\text { comes of using } \\
\text { remdesivir in } \\
\text { patients with } \\
\text { moderate to } \\
\text { severe COVID-19: } \\
\text { A prospective } \\
\text { randomised } \\
\text { study }\end{array}$ & $\begin{array}{l}\text {-Improvement in } \\
\text { clinical outcomes }\end{array}$ & $\begin{array}{l}\text { RDV }+ \text { standard of care }(n=34) \\
\text {-Did not require hospitalization: } 2(5.9 \%) \\
\text { Standard of care }(n=36) \\
\text {-Did not require hospitalization: } 3(8.3 \%)\end{array}$ & 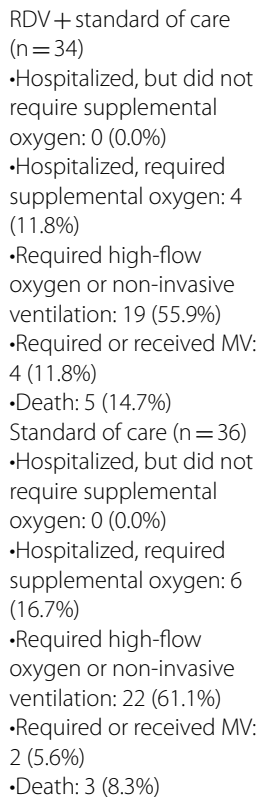 & $\begin{array}{l}\text {-All study cases } \\
\text { were of moder- } \\
\text { ate to severe } \\
\text { disease category } \\
\text {-Did not grade } \\
\text { the adverse } \\
\text { events } \\
\text {-Did not give } \\
\text { placebo injec- } \\
\text { tion in the } \\
\text { no-RDV group } \\
\text {-Not blinded } \\
\text {-Small sample } \\
\text { size }\end{array}$ & $\begin{array}{l}\text { RDV therapy for five } \\
\text { days did not produce } \\
\text { improvement in } \\
\text { clinical outcomes in } \\
\text { moderate to severe } \\
\text { COVID-19 cases } \\
{[2(5.9) \text { vs. } 3 \text { ( } 8.3)} \\
p=0.749]\end{array}$ \\
\hline
\end{tabular}


Table 3 (continued)

\begin{tabular}{|c|c|c|c|c|c|c|}
\hline Author & Study name & Primary endpoint & Primary outcomes & Other outcomes & Limitations & Interpretation \\
\hline $\begin{array}{l}\text { Spinner } \\
\text { et al. [34] }\end{array}$ & $\begin{array}{l}\text { Effect of Remde- } \\
\text { sivir vs Standard } \\
\text { Care on Clinical } \\
\text { Status at } 11 \\
\text { Days in Patients } \\
\text { With Moderate } \\
\text { COVID-19 A } \\
\text { Randomized } \\
\text { Clinical Trial }\end{array}$ & $\begin{array}{l}\text {-Difference in } \\
\text { clinical status } \\
\text { distribution }\end{array}$ & $\begin{array}{l}\text { 10-day RDV }(n=193) \\
\text {-Difference in clinical status distribution vs } \\
\text { standard care: } p=0.18 \\
5 \text {-day RDV ( } n=191) \\
\text {-Difference in clinical status distribution vs } \\
\text { standard care: OR } 1.65 \text { ( } 95 \% \text { Cl: } 1.09,2.48) \text {, } \\
p=0.02\end{array}$ & $\begin{array}{l}\text { 10-day RDV }(\mathrm{n}=193) \\
\text {-D11 clinical status } \\
\text { oDeath: } 2 \text { (1.0\%) } \\
\text { oNot hospitalized: } 125 \\
\text { (64.8\%) } \\
\text { 5-day RDV ( } \mathrm{n}=191) \\
\text {-D11 clinical status } \\
\text { oDeath: } 0 \text { (0.0\%) } \\
\text { oNot hospitalized: } 134 \\
\text { (70.2\%) } \\
\text { Standard care }(\mathrm{n}=200) \\
\text {-D11 clinical status } \\
\text { oDeath: } 4 \text { (2.0\%) } \\
\text { oNot hospitalized: } 120 \\
\text { (60.0\%) }\end{array}$ & $\begin{array}{l}\text {-Original } \\
\text { protocol written } \\
\text { when clinical } \\
\text { understanding } \\
\text { of disease was } \\
\text { limited, so pri- } \\
\text { mary end point } \\
\text { changed on first } \\
\text { day of study } \\
\text { enrollment } \\
\text {-Open-label } \\
\text { design } \\
\text {-Virological out- } \\
\text { comes (SARS- } \\
\text { CoV-2 viral load) } \\
\text { not assessed } \\
\text {-Other lab } \\
\text { parameters that } \\
\text { may have aided } \\
\text { in identifying } \\
\text { predictors of } \\
\text { outcomes not } \\
\text { collected }\end{array}$ & $\begin{array}{l}\text { 5-day course of RDV } \\
\text { improved clinical } \\
\text { status of moderate } \\
\text { COVID-19 patients, } \\
\text { but the magnitude } \\
\text { of treatment was of } \\
\text { questionable clinical } \\
\text { relevance } \\
{[1.65(1.09-2.48) \text { vs. } 1} \\
p=0.02]\end{array}$ \\
\hline $\begin{array}{l}\text { Wang } \\
\text { et al. [36] }\end{array}$ & $\begin{array}{l}\text { Remdesivir in } \\
\text { adults with } \\
\text { severe COVID-19: } \\
\text { a randomised, } \\
\text { double-blind, } \\
\text { placebo-con- } \\
\text { trolled, multicen- } \\
\text { tre trial }\end{array}$ & $\begin{array}{l}\text {-Time to clinical } \\
\text { improvement up } \\
\text { to day } 28 \\
\text {-Defined as the } \\
\text { time from rand- } \\
\text { omization to the } \\
\text { point of a decline } \\
\text { of two levels on a } \\
\text { six-point ordinal } \\
\text { scale of clinical } \\
\text { status (from } \\
1=\text { discharged } \\
\text { to } 6=\text { death) or } \\
\text { discharged alive } \\
\text { from hospital, } \\
\text { whichever came } \\
\text { first }\end{array}$ & $\begin{array}{l}\text { RDV }(n=158) \\
\text {-Time to clinical improvement: } 21.0(13.0,28.0) \\
\text { Control }(n=78) \\
\text {-Time to clinical improvement: } 23.0(15.0,28.0)\end{array}$ & $\begin{array}{l}\text { RDV }(n=158) \\
\text {-Clinical improvement } \\
\text { rates } \\
\text { oD7: } 4(2.5 \%) \\
\text { oD14: } 42(26.6 \%) \\
\text { oD28: } 103(65.2 \%) \\
\text {-D28 mortality: } 22 \text { (13.9\%) } \\
\text {-Duration of IMV, days: } 7.0 \\
\text { (4.0, 16.0) } \\
\text {-Duration of hospital stay, } \\
\text { days: } 25.0 \text { (16.0, 38.0) } \\
\text { Control ( } n=78) \\
\text {-Clinical improvement } \\
\text { rates } \\
\text { oD7: } 2 \text { (2.6\%) } \\
\text { oD14: } 18 \text { (23.1\%) } \\
\text { oD28: } 45 \text { (57.7\%) } \\
\text {-D28 mortality: } 10 \text { (12.8\%) } \\
\text {-Duration of IMV, days: } \\
\text { 15.5 (6.0, } 21.0) \\
\text {-Duration of hospital stay, } \\
\text { days: } 24.0 \text { (18.0, 36.0) }\end{array}$ & $\begin{array}{l}\text { •Insufficient } \\
\text { power to detect } \\
\text { assumed differ- } \\
\text { ences in clinical } \\
\text { outcomes } \\
\text { •Initiation of } \\
\text { treatment late } \\
\text { after symptom } \\
\text { onset } \\
\text {-Frequent use of } \\
\text { corticosteroids } \\
\text { patients may } \\
\text { have promoted } \\
\text { viral replication } \\
\text {-No answer to } \\
\text { whether longer } \\
\text { treatment } \\
\text { course and } \\
\text { higher dose of } \\
\text { RDV would be } \\
\text { beneficial in } \\
\text { patients with } \\
\text { severe COVID-19 }\end{array}$ & $\begin{array}{l}\text { No benefits were } \\
\text { observed with RDV } \\
\text { above and beyond } \\
\text { that observed with } \\
\text { standard therapies } \\
\text { in severe COVID-19 } \\
\text { patients } \\
{[21.0(13.0,28.0) \text { vs. }} \\
23.0(15.0,28.0), 95 \% \\
\text { Cl } 1.23(0.87-1.75)]\end{array}$ \\
\hline $\begin{array}{l}\text { Abba- } \\
\text { spour- } \\
\text { Kasgari } \\
\text { et al. [22] }\end{array}$ & $\begin{array}{l}\text { Evaluation of } \\
\text { the efficacy of } \\
\text { sofosbuvir plus } \\
\text { daclatasvir in } \\
\text { combination } \\
\text { with ribavirin } \\
\text { for hospital- } \\
\text { ized COVID-19 } \\
\text { patients with } \\
\text { moder- } \\
\text { ate disease } \\
\text { compared with } \\
\text { standard care: } \\
\text { a single-centre, } \\
\text { randomized } \\
\text { controlled trial }\end{array}$ & $\begin{array}{l}\text {-Length of hospital } \\
\text { stay }\end{array}$ & $\begin{array}{l}\text { SOF/DCV + ribavirin }(n=24) \\
\cdot \text { Duration of hospitalization, days: } 6(5,7) \\
\text { Standard care }(n=24) \\
\text {-Duration of hospitalization, days: } 6(5.5,7.5)\end{array}$ & 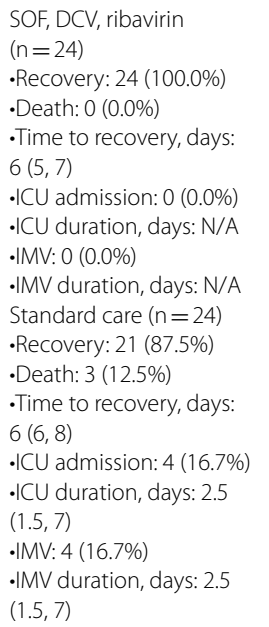 & $\begin{array}{l}\text {-Median age was } \\
\text { higher in the } \\
\text { control arm } \\
\text {-More patients } \\
\text { with diabetes in } \\
\text { the control arm } \\
\text {-Number of } \\
\text { patients not } \\
\text { high enough } \\
\text { to identify } \\
\text { probable ben- } \\
\text { eficial effects on } \\
\text { survival } \\
\text {-Excluded } \\
\text { elderly subject } \\
\text {-Not blinded } \\
\text {-Not able to } \\
\text { analyze biologi- } \\
\text { cal markers of } \\
\text { improvement }\end{array}$ & $\begin{array}{l}\text { There were signs of } \\
\text { improved recovery } \\
\text { and death rates in the } \\
\text { with SOF/DCV + riba- } \\
\text { virin, but the sample } \\
\text { size was too small to } \\
\text { see conclusive differ- } \\
\text { ences } \\
{[6(5-7) \text { vs. } 6 \text { (5.5-7.5) }} \\
p=0.398]\end{array}$ \\
\hline
\end{tabular}


Table 3 (continued)

\begin{tabular}{|c|c|c|c|c|c|c|}
\hline Author & Study name & Primary endpoint & Primary outcomes & Other outcomes & Limitations & Interpretation \\
\hline $\begin{array}{l}\text { Abbass } \\
\text { et al. [46] }\end{array}$ & $\begin{array}{l}\text { Efficacy and } \\
\text { safety of } \\
\text { sofosbuvir plus } \\
\text { daclatasvir or } \\
\text { ravidasvir } \\
\text { in patients with } \\
\text { COVID-19: A } \\
\text { randomized } \\
\text { controlled trial }\end{array}$ & $\begin{array}{l}\text {-Sum of the } \\
\text { counted symp- } \\
\text { toms at D7 and } \\
\text { D10 compared } \\
\text { to D3 } \\
\text { - Mean change } \\
\text { in } \mathrm{SpO}_{2} \text { from D1 } \\
\text { to D10 }\end{array}$ & 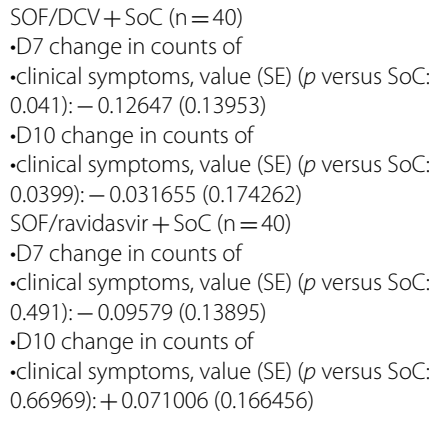 & $\begin{array}{l}\text { SOF/DCV }+\mathrm{SoC}(\mathrm{n}=40) \\
\cdot \mathrm{D} 1 \mathrm{SpO}_{2}: 88.7 \pm 4.2 \\
\cdot \mathrm{D} 10 \mathrm{SpO}_{2}: 95.8 \pm 2.7 \\
\mathrm{SOF} / \mathrm{ravidasvir}+\mathrm{SoC} \\
(\mathrm{n}=40) \\
\cdot \mathrm{D} 1 \mathrm{SpO}_{2}: 87.5 \pm 6.25 \\
\cdot \mathrm{D} 10 \mathrm{SpO}_{2}: 94.52 \pm 4.58 \\
\mathrm{SoC}(\mathrm{n}=40) \\
\cdot \mathrm{D} 1 \mathrm{SpO}_{2}: 87.9 \pm 5.8 \\
\cdot \mathrm{D} 10 \mathrm{SpO}_{2}: 93.4 \pm 3.7\end{array}$ & $\begin{array}{l}\text {-Small sample } \\
\text { size } \\
\text {-Open-label } \\
\text { design } \\
\text {-Lack of a pla- } \\
\text { cebo group }\end{array}$ & $\begin{array}{l}\text { SOF/DCV + SoC was } \\
\text { found to improve } \\
\text { clinical symptoms, } \\
\text { oxygen saturation, and } \\
\text { decrease ICU admis- } \\
\text { sion. SOF/ravidasvir } \\
\text { had no effect relative } \\
\text { to SoC alone }\end{array}$ \\
\hline $\begin{array}{l}\text { El-Bendary } \\
\text { et al. [49] }\end{array}$ & $\begin{array}{l}\text { Efficacy of com- } \\
\text { bined Sofosbuvir } \\
\text { and Daclatasvir } \\
\text { in the treatment } \\
\text { of COVID-19 } \\
\text { patients with } \\
\text { pneumonia: } \\
\text { a multicenter } \\
\text { Egyptian study }\end{array}$ & $\begin{array}{l}\text { - Rate of clinical/ } \\
\text { virological cure }\end{array}$ & $\begin{array}{l}\text { SOF/DCV }(n=96) \\
\text { •Negative PCR D7: 12/24 (50.0\%) } \\
\text {-Negative PCR D14: 81/96 (84.4\%) } \\
\text { Control ( } n=78) \\
\text {-Negative PCR D7: } 9 / 25 \text { (36.0\%) } \\
\text {-Negative PCR D14: } 37 / 78(47.4 \%)\end{array}$ & $\begin{array}{l}\text { SOF/DCV ( } \mathrm{n}=96) \\
\text {-Adm to hospital: } 79 \\
(82.3 \%) \\
\text { •ICU adm: } 19(19.8 \%) \\
\text {-Duration inside hospital, } \\
\text { median (IQR): } 8 \text { (9\%) } \\
\text { •Follow up of WHO } \\
\text { assessment scale, } \\
\text { improved: } 76 \text { (79.2\%) } \\
\text { Control ( } \mathrm{n}=78) \\
\text {-Adm to hospital: } 49 \\
\text { (62.8\%) } \\
\text {-ICU adm: } 24(30.8 \%) \\
\text {-Duration inside hospital, } \\
\text { median (IQR): } 10(12 \%) \\
\text {-Follow up of WHO } \\
\text { assessment scale, } \\
\text { improved: } 57(73.1 \%)\end{array}$ & -Not blinded & $\begin{array}{l}\text { SOF/DCV was effec- } \\
\text { tive as a treatment } \\
\text { for COVID-19 and } \\
\text { was associated with } \\
\text { reduced hospital stay, } \\
\text { a larger proportion of } \\
\text { virological clearance } \\
\text { at Day } 14 \text { and a trend } \\
\text { toward lower mortality } \\
\text { [84.4 vs. } 47.4 p<0.01 \text { ] }\end{array}$ \\
\hline $\begin{array}{l}\text { Khalili } \\
\text { et al. [28] }\end{array}$ & $\begin{array}{l}\text { Efficacy and } \\
\text { safety of sofos- } \\
\text { buvir/ ledipasvir } \\
\text { in treatment of } \\
\text { patients with } \\
\text { COVID-19; A } \\
\text { randomized } \\
\text { clinical trial }\end{array}$ & $\begin{array}{l}\text {-Clinical response } \\
\text { - Time to clinical } \\
\text {-response } \\
\text {-Clinical response } \\
\text {-was defined } \\
\text { as one order } \\
\text { decline in disease } \\
\text { category } \\
\text {-in the five } \\
\text { category ordinal } \\
\text { scale }\end{array}$ & $\begin{array}{l}\text { SOF/LDP }(n=42) \\
\text {-Clinical response: } 38(90.5 \%) \\
\text {-Time to clinical response, days: } 2(1,3.75) \\
\text { Control }(n=40) \\
\text {-Clinical response: } 37(92.5 \%) \\
\text {-Time to clinical response, days: } 4(2,5)\end{array}$ & $\begin{array}{l}\text { SOF/LDP }(n=42) \\
\text {-Duration of hospital stay, } \\
\text { days: } 4(2,9.5) \\
\text {-Duration of ICU stay, } \\
\text { days: } 6(4,11) \\
\text {-14-day mortality: } 3 \\
(8.8 \%) \\
\text { Control }(n=40) \\
\text {-Duration of hospital stay, } \\
\text { days: } 5(3.25,7) \\
\text {-Duration of ICU stay, } \\
\text { days: } 9(6,12) \\
\text { - } 14-\text { day mortality: } 3 \\
\text { (7.5\%) }\end{array}$ & $\begin{array}{l}\text {-Not blinded } \\
\text {-Follow-up RT- } \\
\text { PCR and chest } \\
\text { imaging were } \\
\text { not possible } \\
\text {-Small sample } \\
\text { size }\end{array}$ & $\begin{array}{l}\text { SOF/LDP accelerated } \\
\text { time to } \\
\text { the clinical response, } \\
\text { but did not have a } \\
\text { significant effect on } \\
\text { duration of hospital } \\
\text { stay or mortality } \\
\text { Clinical Response: [38 } \\
\text { ( } 90.48) \text { vs. } 37 \text { (92.5) } \\
\text { p=0.65] } \\
\text { Time to clinical } \\
\text { response (days): [2 } \\
(1-3.75) \text { vs. } 4 \text { (2.5) } \\
p=0.02]\end{array}$ \\
\hline $\begin{array}{l}\text { Roozbeh } \\
\text { et al. [32] }\end{array}$ & $\begin{array}{l}\text { Sofosbuvir and } \\
\text { daclatasvir for } \\
\text { the treatment } \\
\text { of COVID-19 } \\
\text { outpatients: a } \\
\text { double-blind, } \\
\text { randomized } \\
\text { controlled trial }\end{array}$ & $\begin{array}{l}\text {-Symptom allevia- } \\
\text { tion after } 7 \text { days of } \\
\text { follow-up }\end{array}$ & $\begin{array}{l}\text { SOF/DCV + standard care }(n=27) \\
\text {-Any symptoms: } \\
\text {-D1: } 27(100.0 \%) \\
\text {-D3: } 16(59.3 \%) \\
\text {-D5: } 12(44.4 \%) \\
\text {-D7: } 7(25.9 \%) \\
\text { Standard care }(n=28) \\
\text {-Any symptoms: } \\
\text {-D1: } 26(92.9 \%) \\
\text {-D3: } 15(53.6 \%) \\
\text {-D5: } 12(42.9 \%) \\
\text {-D7: } 7(25.0 \%)\end{array}$ & $\begin{array}{l}\text { SOF/DCV + standard care } \\
(n=27) \\
\text { •Hospital admission: } 1 \\
(3.7 \%) \\
\text { •Fatigue D30: } 2(7.4 \%) \\
\text {-Anosmia D30: } 0(0.0 \%) \\
\text {-Dyspnea D30: } 4(14.8 \%) \\
\text { Standard care }(n=28) \\
\text { •Hospital admission: } 4 \\
\text { (14.3\%) } \\
\text { •Fatigue D30: } 16 / 26 \\
(61.5 \%) \\
\text {-Anosmia D30: } 3 / 26 \\
\text { (11.5\%) } \\
\text {-Dyspnea D30: } 11 / 26 \\
\text { (42.3\%) }\end{array}$ & $\begin{array}{l}\text {-Assessment } \\
\text { of symptom } \\
\text { outcomes not } \\
\text { carried out using } \\
\text { an objective } \\
\text { grading system } \\
\text {-Small sample } \\
\text { size }\end{array}$ & $\begin{array}{l}\text { SOF/DCV did not } \\
\text { significantly reduce } \\
\text { symptoms at } 7 \text { days } \\
\text { compared to control. } \\
\text { However, the interven- } \\
\text { tion significantly } \\
\text { reduced the number } \\
\text { of patients with } \\
\text { fatigue and dyspnea } \\
\text { at } 1 \text { month } \\
\text { [7 (26) vs. } 7 \text { (28) } \\
p=1.00]\end{array}$ \\
\hline
\end{tabular}


Table 3 (continued)

\begin{tabular}{|c|c|c|c|c|c|c|}
\hline Author & Study name & Primary endpoint & Primary outcomes & Other outcomes & Limitations & Interpretation \\
\hline $\begin{array}{l}\text { Sadeghi } \\
\text { et al. [33] }\end{array}$ & $\begin{array}{l}\text { Sofosbuvir and } \\
\text { daclatasvir } \\
\text { compared with } \\
\text { standard of care } \\
\text { in the treatment } \\
\text { of patients } \\
\text { admitted to } \\
\text { hospital with } \\
\text { moderate or } \\
\text { severe corona- } \\
\text { virus infection } \\
\text { (COVID-19): a } \\
\text { randomized } \\
\text { controlled trial }\end{array}$ & $\begin{array}{l}\text {-Clinical recovery } \\
\text { within } 14 \text { days of } \\
\text { treatment }\end{array}$ & $\begin{array}{l}\text { SOF/DCV }+ \text { standard care }(n=33) \\
\cdot \text { Clinical recovery } \leq 14 \text { days: } 29(87.9 \%) \\
\text { Standard care }(n=33) \\
\text {-Clinical recovery } \leq 14 \text { days: } 22(66.7 \%)\end{array}$ & $\begin{array}{l}\text { SOF/DCV + standard care } \\
(\mathrm{n}=33) \\
\text {-Duration of hospitaliza- } \\
\text { tion, days: } 6(4,8) \\
\text {-Time to clinical recovery, } \\
\text { days: } 6(4,10) \\
\text {-IMV: } 3(9.1 \%) \\
\text {-Death: } 3(9.1 \%) \\
\text { Standard care }(\mathrm{n}=33) \\
\text {-Duration of hospitaliza- } \\
\text { tion, days): } 8(5,13) \\
\text {-Time to clinical recovery, } \\
\text { days: } 11(6,17) \\
\text {-IMV: } 7(21.2 \%) \\
\text {-Death: } 5(15.2 \%)\end{array}$ & $\begin{array}{l}\text {-Not blinded } \\
\text {-Fewer patients } \\
\text { in the treatment } \\
\text { arm received } \\
\text { LVP/r } \\
\text {-Small sample } \\
\text { size }\end{array}$ & $\begin{array}{l}\text { SOF/DCV significantly } \\
\text { reduced the duration } \\
\text { of hospital stay } \\
{[29 \text { (88) vs. } 22 \text { (67) }} \\
p=0.076]\end{array}$ \\
\hline $\begin{array}{l}\text { Sayad } \\
\text { et al. [43] }\end{array}$ & $\begin{array}{l}\text { Efficacy and } \\
\text { safety of sofos- } \\
\text { buvir/velpatasvir } \\
\text { versus the } \\
\text { standard of } \\
\text { care in adults } \\
\text { hospitalized } \\
\text { with COVID-19: } \\
\text { a single-centre, } \\
\text { randomized } \\
\text { controlled trial }\end{array}$ & -28-day mortality & $\begin{array}{l}\text { SOFNEL }(n=40) \\
\text {-All-cause mortality: } 3(7.5 \%) \\
\text { Control ( } n=40) \\
\text {-All-cause mortality: } 3(7.5 \%)\end{array}$ & $\begin{array}{l}\text { SOFNEL ( } n=40) \\
\text {-Time to clinical improve- } \\
\text { ment, days: } 6(4,8) \\
\text {-Duration of hospital stay, } \\
\text { days: } 6(5,8.5) \\
\text {-Time from randomiza- } \\
\text { tion to death, days: } 6 \\
(2,9) \\
\text {-Need for MV: } 1 \text { ( } 2.4 \%) \\
\text {-Duration of MV-days: } \\
3 \text { (3,3) } \\
\text {-RT-PCR conversion } \\
\text { (positive to negative): } 6 \\
\text { (15.0\%) } \\
\text { Control (N=40) } \\
\text {-Time to clinical improve- } \\
\text { ment, days: } 7 \text { (4-11) } \\
\text {-Duration of hospital stay, } \\
\text { days: } 7 \text { (5-13) } \\
\text {-Time from randomiza- } \\
\text { tion to death, days: } 7 \\
\text { (7, 30) } \\
\text {-Need for MV: } 3 \text { (8.1\%) } \\
\text { •Duration of MV, days: } \\
1 \text { (1, } 1 \text { ) } \\
\text {-RT-PCR conversion } \\
\text { (positive to negative): } 4 \\
\text { (10.0\%) }\end{array}$ & $\begin{array}{l}\text {-Did not assess } \\
\text { viral load } \\
\text {-Small sample } \\
\text { size } \\
\text {-Open-label } \\
\text { design }\end{array}$ & $\begin{array}{l}\text { SOFNEL + SoC did not } \\
\text { improve the clinical } \\
\text { status or reduce mor- } \\
\text { tality in patients with } \\
\text { moderate to severe } \\
\text { COVID-19 } \\
{[3(7.5) \text { vs. } 3(7.5)} \\
p=1.00]\end{array}$ \\
\hline $\begin{array}{l}\text { Hol- } \\
\text { ubovska } \\
\text { et al. [50] }\end{array}$ & $\begin{array}{l}\text { Enisamium is an } \\
\text { inhibitor of the } \\
\text { SARS-CoV-2 RNA } \\
\text { polymerase and } \\
\text { shows improve- } \\
\text { ment of recovery } \\
\text { in COVID-19 } \\
\text { patients in an } \\
\text { interim analysis } \\
\text { of a clinical trial }\end{array}$ & $\begin{array}{l}\text {-Time-to-recovery } \\
\text {-Defined as } \\
\text { improvement in } \\
\text { the Severity Rat- } \\
\text { ing (SR) baseline } \\
\text { status by } 2 \text { SR } \\
\text { score values (e.g., a } \\
\text { change from SR } 4 \\
\text { to SR 6) }\end{array}$ & $\begin{array}{l}\text { Enisamium }(\mathrm{n}=\sim 186) \\
\text {-Mean time-to- } \\
\text { •recovery, days: } 11.1 \\
\text { Placebo }(\mathrm{n}=\sim 186) \\
\text {-Mean time-to- } \\
\text {-recovery, days: } 13.9 \text { days }\end{array}$ & $\begin{array}{l}\text { Enisamium }(\mathrm{n}=\sim 186) \\
\text {-Maximum time-to- } \\
\text { recovery, days: } 21 \\
\text { Placebo ( } \mathrm{n}=\sim 186) \\
\text {-Maximum time-to- } \\
\text { recovery, days: not } \\
\text { reported }\end{array}$ & $\begin{array}{l}\text { Patient baseline } \\
\text { characteristics } \\
\text { not reported } \\
\text {-Group sizes not } \\
\text { directly reported }\end{array}$ & $\begin{array}{l}\text { Enisamium treatment } \\
\text { shortens the time to } \\
\text { recovery for COVID-19 } \\
\text { patients needing } \\
\text { oxygen } \\
\text { [13.9 vs. } 11.1 \\
p=0.0259 \text { ] }\end{array}$ \\
\hline
\end{tabular}

Data are presented as mean \pm standard deviation or median (IQR) unless otherwise stated

*Statistically different from comparator

$A d m=$ admission; $A L T=$ alanine aminotransferase; $A O T=$ ambulatory oxygen therapy; $A R B=$ umifenovir (Arbidol); $A S T=$ aspartate aminotransferas; $B / M$ $=$ baloxavir/marboxil; $C Q=$ chloroquine; $C T=$ computed tomography; $D \#=$ day $\# ; D B=$ double-blind; $D C V=$ daclatasvir; $E C M O=$ extracorporeal membrane oxygenation; $F V P=$ favipiravir; $G I=$ gastrointestinal; $H C Q=$ hydroxychloroquine; $H R=$ hazard ratio; $I C U=$ intensive care unit; $I F N=$ interferon; $I M V=$ invasive mechanical ventilation; $L D P=$ ledipasvir; $L P V / r=$ lopinavir/ritonavir; $M V=$ mechanical ventilation; $N M V=$ non-invasive mechanical ventilation; $O L=$ open-label; $O R$ $=$ odds ratio; $\mathrm{PaO} 2 / \mathrm{FiO} 2=$ arterial partial pressure of oxygen/fraction of inspired oxygen ratio; $Q T C=$ corrected $\mathrm{QT}$ interval; $R D V=\mathrm{Remdesivir} ; R C T=$ randomized controlled trial; $R T-P C R=$ reverse transcriptase polymerase chain reaction; $r x n=$ reaction; $S o C=$ standard of care; $S O F=$ sofosbuvir; $S p O 2=$ oxygen saturation; $V E L$ $=$ velpatasvir 
improvement of chest imaging findings $(\mathrm{p}=0.029)$ and time to conversion to negative RT-PCR $(\mathrm{p}=0.041)$ were significantly shorter in the favipiravir group compared to the placebo group, while temperature $(\mathrm{p}=0.18)$ and $\mathrm{SpO}_{2}$ $(\mathrm{p}=0.51)$ showed no significant difference (Table 3$)$.

Udwadia et al. conducted a multicentric, open-label trial to compare favipiravir to standard supportive care alone [35]. No significant difference was found in time to conversion to negative RT-PCR tests $(\mathrm{p}=0.1290)$ or duration of hospital stay $(\mathrm{p}=0.1079)$. However, the favipiravir group had a significantly shorter time to resolution of clinical symptoms (3 days [95\% CI: 3-4 days]) compared to the control group (5 days [95\% CI: $4-6$ days], $\mathrm{p}=0.030$ ).

Doi et al. [48] conducted a multicentric, open-label trial to compare patients treated with favipiravir starting on either day 1 (early) or day 6 (late) after their hospital admission. Patients received favipiravir for up to 10 days. Treatment could be discontinued after 6 days if their symptoms had resolved and they had two consecutive negative RT-PCR tests, meeting the requirements to be discharged from the hospital. Favipiravir did not significantly affect viral clearance by day 6 (HR: 1.416 [95\% CI 0.764-2.623]). However, early treatment did lead to a significantly higher chance of viral clearance at day 6 in patients who were enrolled in the study more than three days after their first positive RT-PCR test (HR: 2.829 [95\% CI 1.198-6.683]), indicating that there may be a window after infection where initiating treatment is more effective.

Chen et al. compared favipiravir with umifenovir in COVID-19 patients [85] in a multicentric, open-label trial. Umifenovir is an antiviral drug that prevents cell attachment and viral entrance by trimerization of the SARS-CoV-2 spike glycoprotein. This blockade forms a naked or immature virus that less contagious [86]. Patients also received standard therapy, which consisted of antivirals, steroids, traditional Chinese herbal medicines, immunomodulatory drugs, steroids, antibiotics, psychotic drugs, nutritional supplements, and oxygen support. The primary outcome was rate of clinical recovery at day 7. Secondary outcomes were all-cause mortality, dyspnea, respiratory failure, auxiliary oxygen therapy or noninvasive mechanical ventilation (NMV), latency to pyrexia and cough relief, and need for intensive care. While no differences were found in clinical recovery (favipiravir 61.2\% [71/116]; umifenovir 51.7\% [62/120]; $P=0.1396)$ or in most secondary outcomes between treatments, favipiravir did shorten the latency of pyrexia and cough relief.

Several trials did not find significant differences between treatment with favipiravir and their various comparator groups. Lou et al. conducted an open-label, single-center trial to evaluate the clinical outcomes and plasma concentrations of baloxavir acid and favipiravir in COVID-19 patients [30]. Patients were randomly assigned to one of three groups: a baloxavir marboxil group, a favipiravir group, and a control group, which included umifenovir. Median times from randomization to clinical improvement, viral negativity at day 7 , and viral negativity at day 14 were similar between the three groups (Table 3). One patient in the baloxavir marboxil group and two patients in the favipiravir group were transferred to the ICU within 7 days due to declines in oxygen index or progressive disease on computed tomography $(\mathrm{CT})$. One patient in the baloxavir marboxil group required extracorporeal membrane oxygenation (ECMO) support after 10 days.

Dabbous et al. conducted a multicentric trial comparing favipiravir and chloroquine (CQ) in patients with confirmed cases of COVID-19 [41]. There were no significant differences between the groups in mortality $(p=1.00)$, duration of hospital stay $(\mathrm{p}=0.060)$, mechanical ventilation $(\mathrm{p}=0.118)$, or oxygen saturation $(\mathrm{p}=0.129)$. Bosaeed et al. also compared favipiravir (10 days) and HCQ [40]. Nearly half of the favipiravir group discontinued therapy before the end of the trial due to pill burden or personal preference. This study found no significant difference in conversion to negative RT-PCR tests $(p=0.73)$, time to clinical improvement $(\mathrm{p}=0.29)$, duration of hospital stay $(\mathrm{p}=0.42), 28$-day mortality $(\mathrm{p}=0.45)$, and 90-day mortality $(p=0.91)$. Solaymani-Dodaran et al. conducted a multicentric, open-label trial to compare favipiravir (in addition to $\mathrm{HCQ}$ ) to $\mathrm{LPV} / \mathrm{r}$ [44]. They found no significant differences between the groups for mortality $(p=0.52)$, transfer to the ICU $(p=0.47)$, time to clinical recovery $(\mathrm{p}=0.54)$, incidence of clinical recovery (HR: 0.94 [95\% CI 0.75-1.17]), or change in oxygen saturation $(\mathrm{p}=0.46)$.

\section{Lopinavir/Ritonavir}

$\mathrm{LPV} / \mathrm{r}$ is an HIV-1 protease inhibitor combination. Ritonavir is combined with lopinavir to increase the latter's plasma half-life by inhibiting cytochrome P450 [87]. $\mathrm{LPV} / \mathrm{r}$ is approved by the FDA for treatment of HIV-1 infection in adult and pediatric patients [88]. LPV/r has also exhibited efficacy to treat influenza, severe acute respiratory syndrome (SARS), and Middle Eastern respiratory syndrome (MERS) infection [89-91]. Nine RCTs included LPV/r for COVID-19 therapy: two large trials (RECOVERY [26] and TOGETHER [27]), and seven relatively smaller trials $(n=86-664)$ [24, 31, 37, 40, 44, 47]. The trial conducted by Solaymani-Dodaran et al. compared LPV/r to favipiravir and found no significant differences, as discussed in the Favipiravir section above [44]. Similarly, none of the other trials identified a significant 
positive effect of LPV/r on outcomes in COVID-19 patients.

The RECOVERY trial was an open-label, platform trial conducted between March 19, 2020 and June 29, 2020 among 176 hospitals in the United Kingdom (UK). Patients were randomized to either standard of care alone or standard of care plus oral LPV/r for 10 days or until discharge. The primary outcome was 28-day all-cause mortality, which did not significantly differ between the intervention and control groups (rate ratio [RR] 1.03, 95\% CI $0.91-1.17 ; P=0.60$ ), and the results were consistent among all pre-specified subgroups. There was also no difference in the time until discharge alive or proportion of patients discharged alive within 28 days (RR $0.98,95 \%$ CI $0.91-1.05 ; P=0.53$ ). Additionally, there was no difference in the proportion of patients who met the composite endpoint of invasive mechanical ventilation or death among patients who were not on invasive mechanical ventilation at baseline (RR 1.09, 95\% CI 0.99-1.20; $P=0.092$ ).

The TOGETHER trial was conducted between June 2, 2020 and September 20, 2020 in Brazil [27]. The trial compared LPV/r to HCQ or placebo. The trial was discontinued early after finding no significant difference between the groups in COVID-19-associated hospitalization (LPV/r: HR, 1.16 [95\% CI, 0.53-2.56]) or viral clearance at day $14(\mathrm{LPV} / \mathrm{r}$ : odds ratio [OR], 1.04 [95\% CI, 0.94-1.16]). Incidence of mortality was similar between the LPV/r and placebo groups. Ader et al. also compared $\mathrm{LPV} / \mathrm{r}$ to HCQ and control, in addition to LPV/r with IFN- $\beta-1 \mathrm{a}$, and discontinued the LPV/r and HCQ arms early due to lack of significant difference in clinical status at day 15 compared to control [37]. Arabi et al. also conducted a randomized, multicentric trial comparing $\mathrm{LPV} / \mathrm{r}, \mathrm{HCQ}$, or a combination to a control group with no antiviral therapy [40]. They found a $98.5 \%$ probability of harm compared to control for LPV/r alone based on inhospital mortality.

Cao et al. conducted an open-label trial comparing $\mathrm{LPV} / \mathrm{r}$ to standard of care in patients with SARS-CoV-2 infection and hypoxia [24]. There was no difference in time to clinical improvement (HR 1.24, 95\% CI 0.90$1.72)$ or mortality at 28 days $(19.2 \%$ vs. $25.0 \%$; mean difference -5.8, 95\% CI -17.3-5.7). The LPV/r group had a shorter median time to clinical improvement by one day compared to standard care alone on a modified intention-to-treat analysis (HR 1.39, 95\% CI 1.00-1.91).

Three studies compared umifenovir to LPV/r [31, 47]. $\mathrm{Li}$ et al. conducted an exploratory trial to study the efficacy and safety of LPV/r versus umifenovir in patients with mild to moderate COVID-19 [31]. There were no differences in positive-to-negative conversion of SARSCoV-2 RT-PCR tests on days 7 and 14. Also, there were no differences in mean time to test conversion (9.0, 9.1, and 9.3 days; $P=0.981$ ) or in the conversion rate from moderate to severe/critical clinical status $(23.5 \%, 8.6 \%$, and $11.8 \% ; P=0.206)$ among $L P V / r$, umifenovir, and control groups, respectively.

Nojomi et al. investigated the efficacy of umifenovir compared to $\mathrm{LPV} / \mathrm{r}$ in COVID-19 patients [31]. The patients were randomized to receive umifenovir or $\mathrm{LPV} / \mathrm{r}$ for 7-14 days, based on disease severity, as well as HCQ on day 1. Patients that received umifenovir had a shorter duration of hospitalization (7.2 days) compared to patients that received LPV/r (9.6 days, $P=0.02$ ). Moreover, $81 \%$ of patients in the umifenovir group had mild involvement on chest $\mathrm{CT}$ after 30 days of admission compared to $53 \%$ in the $L P V / r$ group $(P=0.004)$.

Alavi Darazam et al. compared a combination of LPV/r, $\mathrm{HCQ}$, and IFN- $\beta 1 \mathrm{a}$ with and without umifenovir in a single-center, open-label trial [47]. All patients received $\mathrm{LPV} / \mathrm{r}, \mathrm{HCQ}$, and IFN- $\beta 1 \mathrm{a}$. Half of the patients also received umifenovir. The groups did not have a significant difference in mortality $(\mathrm{p}=0.62)$ or time to clinical improvement $(\mathrm{p}=0.22)$, defined as improvement by two points on a seven-category ordinal scale. No significant difference in mortality was found between the groups when adjusted for time between symptom onset and trial enrollment either (presentation $\leq 7$ days from symptom onset, $\mathrm{p}=0.49 ;>7$ days, $\mathrm{p}=1.00$ ), indicating that starting treatment earlier is unlikely to affect the efficacy of combining umifenovir with $\mathrm{LPV} / \mathrm{r}$ and other treatments.

\section{Remdesivir}

Remdesivir is an RNA-dependent RNA polymerase inhibitor with in-vitro activity demonstrated against SARS-CoV-2 and MERS-CoV [34, 92]. It is FDAapproved for COVID-19 treatment in adult and pediatric patients (12 years or older and weighing at least $40 \mathrm{~kg}$ ) requiring hospitalization [93]. We identified six trials used remdesivir to treat COVID-19. Three trials found significant differences between the remdesivir treatment and comparator groups $[23,34,53]$ and three did not [36, 39, 51].

The Adaptive Covid-19 Treatment Trial (ACTT-1) was a multicentric, double-blind, placebo-controlled trial of remdesivir in patients with severe COVID-19 pneumonia [23]. Median recovery times were lower in the remdesivir group, with a rate ratio for recovery of 1.29 (95\% CI $1.12-1.49, P<0.001)$. The patients who received remdesivir were more likely to have clinical improvement by day 15 when compared to placebo (OR 1.5, 95\% CI 1.2-1.9, after adjustment for actual disease severity). The KaplanMeier estimates of mortality at days 15 and 29 were $6.7 \%$ and $11.4 \%$ in the remdesivir group and $11.9 \%$ and $15.2 \%$ in the control group, respectively. 
Spinner et al. compared remdesivir to standard of care in a multicentric, open-label trial of hospitalized patients with moderate COVID-19 pneumonia [34]. Patients were randomized to receive remdesivir for 5 or 10 days or standard care alone. On day 11, the odds for a better clinical status distribution were greater in the 5-day remdesivir group as compared to the standard care group (OR $1.65,95 \%$ CI 1.09-2.48; $P=0.02$ ) but was not significant between 10-day remdesivir and standard care groups ( $P=0.18$ by Wilcoxon Rank Sum test). Mortality at day 28 was $1 \%, 2 \%$, and $2 \%$ in 5 -day remdesivir, 10 -day remdesivir, and standard care groups, respectively.

Goldman et al. also compared five- and ten-day courses of remdesivir [53]. Their open-label, phase 3 trial included patients with confirmed SARS-CoV-2 infection, $\mathrm{SpO}_{2}$ of $\leq 94 \%$ on room air, and radiologic evidence of pneumonia. The patients randomized to the 10-day group had significantly worse clinical status than those in the 5-day group, as assessed on a seven-category ordinal scale $(\mathrm{p}=0.02)$. Discharge rates were higher in patients whose symptoms started less than 10 days before receiving the first dose of remdesivir (62\%) than in those whose symptoms started 10 or more days before their first dose (49\%), indicating that regardless of drug regimen, there may be advantages to starting remdesivir earlier.

Several trials found no significant effect of remdesivir on patient outcomes. Wang et al. conducted a doubleblind, placebo-controlled, multicenter trial in COVID19 patients with $\mathrm{SpO}_{2} \leq 94 \%$ in room air or $\mathrm{PaO}_{2} / \mathrm{FiO}_{2}$ ratio $\leq 300 \mathrm{mmHg}$ and radiological evidence of pneumonia [36]. Patients were assigned to remdesivir or placebo, along with standard of care. There was no difference in time to clinical improvement with remdesivir as compared to placebo (HR 1.23, 95\% CI 0.87-1.75). Time to clinical improvement in a subgroup of patients with symptom duration $\leq 10$ days was not significantly different with remdesivir compared to placebo (HR 1.52, 95\% CI 0.95-2.43).

Mahajan et al. conducted a trial comparing remdesivir to standard of care in patients over 40 years old with moderate to severe COVID-19, but not on mechanical ventilation [51]. Clinical status was assessed with a sixpoint ordinal scale based on need for oxygen supplementation and ventilation, hospitalization and mortality status. The groups showed no significant difference in clinical status at day 24, including hospitalization and mortality $(\mathrm{p}=0.749)$, despite the potential bias towards the remdesivir group found in the risk of bias assessment (Additional file 1). Discharge rates were higher for patients who received treatment less than 5 days after symptom onset regardless of treatment group. BarrattDue et al. also conducted a RCT comparing remdesivir, $\mathrm{HCQ}$, or standard of care alone and found no significant differences between the groups for in-hospital mortality (HR: 1.0 [95\% CI 0.4-2.9]) and the groups had similar rates of viral clearance [39].

\section{Sofosbuvir/Daclatasvir}

Sofosbuvir and daclatasvir are antiviral agents that inhibit viral RNA replication via NS5A and NS5B polymerase inhibition, respectively [94, 95]. Sofosbuvir and daclatasvir are FDA-approved for treatment of chronic hepatitis C [51]. SARS-CoV-2 possesses similar mechanisms of RNA replication as observed in other RNA viruses; as such, sofosbuvir and daclatasvir combined may demonstrate efficacy to inhibit SARS-CoV-2 replication [22, 96, 97]. We identified seven RCTs that used sofosbuvir and daclatasvir or a combination of sofosbuvir and other drugs to treat COVID-19. Of the RCTs that used sofosbuvir/daclatasvir, all five reported significantly better results for the treatment group for at least one outcome, although the magnitude of the effect was often small $[22,32,33,46,49]$. Of the three RCTs that included sofosbuvir combined with drugs other than daclatasvir, none reported significant differences between the treatment and control groups $[28,43,46]$.

Sadeghi et al. conducted a phase 3, multicenter trial to compare the effects of sofosbuvir/daclatasvir with standard of care versus standard of care alone (HCQ and LPV/r at physician discretion) in moderate to severe COVID-19 patients [33]. Sofosbuvir/daclatasvir was started later than treatment in the control arm due to delays in receiving RT-PCR reports. Clinical recovery within 14 days from enrollment was achieved in $88 \%$ $(29 / 33)$ of patients in the sofosbuvir/daclatasvir arm and $67 \%(22 / 33)$ of patients in the control arm $(P=0.076)$. Patients in the sofosbuvir/daclatasvir group experienced shorter hospital stays than patients in the control group (6 [4-8] days vs. 8 [5-13] days, respectively; $P=0.029$ ), and the sofosbuvir/daclatasvir group exhibited a higher cumulative incidence of hospital discharge as compared to the control group (Gray's $P=0.041$ ). All-cause mortality was similar between groups.

Abbaspour Kasgari et al. conducted a single-center trial to evaluate the efficacy of sofosbuvir/daclatasvir in combination with ribavirin compared to standard of care (including other antivirals) for hospitalized patients with moderate COVID-19 [22]. Secondary outcomes included the frequency of ICU admission, duration of ICU admission, the frequency and time to recovery, mechanical ventilation, and invasive mechanical ventilation. There were no statistically significant differences in secondary outcomes between the two groups except for cumulative incidence of recovery (Gray's $P=0.033$ ), which was higher in the sofosbuvir/daclatasvir arm. 
Roozbeh et al. investigated the efficacy of sofosbuvir/ daclatasvir combined with HCQ for the treatment of COVID-19 outpatients compared to HCQ and standard of care using a double-blinded trial [32]. There was no difference between groups in the primary endpoint of symptom alleviation at day 7 follow-up or in the secondary endpoint of hospital admission (1 patient hospitalized in treatment group, 4 hospitalized in control group). Two patients in the sofosbuvir/daclatasvir arm reported fatigue at 1 month follow-up, while 16 patients reported fatigue in the control arm $(P<0.001)$. Dyspnea at 30-day follow-up was less common in the sofosbuvir/ daclatasvir arm $(14.8 \%$ [4/27]) than in the control arm (42.3\% [11/26], $P=0.035)$.

El-Bendary et al. conducted a multi-centric trial comparing sofosbuvir/daclatasvir combined with HCQ to HCQ alone [49]. Patients treated with sofosbuvir/ daclatasvir had a significantly lower median duration of hospitalization (8 days vs. 10 days in control group, $\mathrm{p}<0.01$ ) and a higher incidence of negative RT-PCR tests at day 14 , with $84 \%(81 / 96)$ negative compared to $47 \%(37 / 78)$ negative in the control group $(\mathrm{p}<0.01)$. The groups showed no significant differences in mortality $(\mathrm{p}=0.07)$, ICU admission $(\mathrm{p}=0.10)$, and clinical improvement on a seven-category ordinal scale $(p=0.07)$. The risk of bias assessment identified potential bias in favor of the sofosbuvir/daclatasvir group, but the potential bias was not expected to fully account for the effect observed (Additional file 1).

Abbass et al. compared sofosbuvir/daclatasvir to standard of care, with all patients receiving additional therapies, such as $\mathrm{HCQ}$, ivermectin, $\mathrm{LPV} / \mathrm{r}$, or remdesivir, at the treating physician's discretion [46]. Patients receiving sofosbuvir/daclatasvir showed significant clinical improvement compared to standard of care on both day $7(\mathrm{p}=0.041)$ and day $10(\mathrm{p}=0.040)$, as measured by the number of clinical symptoms experienced relative to day 3. The sofosbuvir/daclatasvir group also showed significant improvement in $\mathrm{SpO}_{2}(91.3 \% \pm 4.7 \%)$ compared to the standard of care group $(87.4 \% \pm 8.8 \%, \mathrm{p}=0.016)$ starting on day 4 and continuing until the data collection ended on day 10. The groups did not have significant differences in incidence of viral clearance $(\mathrm{p}=0.581)$, ICU admission $(\mathrm{p}=0.254)$, or mortality $(\mathrm{p}=0.329)$.

Three RCTs combined sofosbuvir with other drugs. Abbass et al. included sofosbuvir/ravidasvir along with sofosbuvir/daclatasvir [46]. They found no significant difference between sofosbuvir/ravidasvir and standard of care in clinical improvement $(\mathrm{p}=0.66969)$, oxygen saturation $(\mathrm{p}=0.054)$, viral clearance $(\mathrm{p}=0.893)$, ICU admission $(\mathrm{p}=0.254)$, or mortality at day $10(\mathrm{p}=0.329)$. Khalili et al. compared sofosbuvir/ledipasvir to standard of care alone [28]. They found that sofosbuvir/ledipasvir had a shorter time to clinical improvement (2 [1-3.75]) compared to control $(4[2-5, p=0.02)$, but no significant differences in incidence of clinical improvement $(p=0.65)$, duration of hospital stay $(\mathrm{p}=0.98)$, or 14 -day mortality $(p=0.60)$ between the groups. Sayad et al. compared sofosbuvir/velpatasvir to standard of care alone [43]. They likewise found no difference in 28-day mortality $(\mathrm{p}=0.38)$, time to clinical improvement (HR: $1.2[95 \%$ CI 0.6-2.2], $\mathrm{p}=0.30$ ), or conversion to negative RT-PCR tests $(\mathrm{p}=0.49)$.

\section{Enisamium}

One study evaluated the efficacy of enisamium, an antiviral drug whose metabolite is a viral RNA polymerase inhibitor [98]. Holubovska et al. conducted a doubleblind, placebo-controlled, phase 3 trial comparing enisamium to a placebo [50]. No differences in time to recovery was found overall or among patients who did not initially require oxygen. However, among patients who did require oxygen supplementation when enrolled, enisamium decreased the recovery time (11.1 days) compared to the placebo group (13.9 days, $\mathrm{p}=0.0259)$. All patients in the enisamium group recovered by day 21 , while not all patients in the placebo group recovered before data collection for interim analysis ended on day 29.

\section{Discussion}

Here, we examined the results of RCTs that investigated the efficacy of antiviral drugs for the treatment of COVID-19. While clinical trials of new antiviral candidates are ongoing, current evidence suggests that the success of antiviral therapy for COVID-19 treatment is dependent on multiple factors, including time from symptom onset to treatment.

Of the antiviral therapies we reviewed, the antiviral combination of sofosbuvir/daclatasvir most consistently exhibited efficacy for COVID-19 treatment across some clinical outcomes, although study sizes were small, and results were often inconsistent [22, 32, 33, 46, 49]. Inclusion criteria for COVID-19 severity varied between studies, which may account for some of the inconsistency. In the largest sofosbuvir RCT, consisting of 174 patients, ElBendary et al. reported that patients treated with sofosbuvir/daclatasvir had a lower duration of hospitalization and higher incidence of viral clearance [49]. Other studies reported positive effects of sofosbuvir/daclatasvir, but which outcomes were reported varied [22, 33, 46]. However, Roozbeh et al. did not observe a difference in symptoms between groups with mild COVID-19 after 7 days of treatment [32], and there were no mortality benefits observed with sofosbuvir/daclatasvir treatment. Additionally, combinations of sofosbuvir with other drugs 
similar to daclatasvir did not lead to differences in outcomes compared to standard of care [28, 43, 46]. The fact that sofosbuvir/daclatasvir is available in pill form as opposed to IV (as is the case with remdesivir), its inexpensive price tag (14-day treatment is \$4.42 USD) [99], and its favorable safety profile noted in hepatitis $\mathrm{C}$ treatment $[100,101]$ make sofosbuvir/daclatasvir an appealing option, provided its efficacy can be established in larger RCTs.

While remdesivir had shown early promise for effective treatment of COVID-19, the trials here demonstrated differing results. A previous meta-analysis found that remdesivir treatment of COVID-19 resulted in lower odds for mechanical ventilation or ECMO (OR 0.48, 95\% CI 0.34, $0.69)$ and higher odds for hospital discharge at 28 days (OR 1.44, 95\% CI 1.16, 1.79), while odds for mortality (OR $0.77,95 \%$ CI $0.56,1.06)$ were the same with or without remdesivir treatment [102]. Another meta-analysis found that remdesivir did not have a significant effect on the time to clinical improvement, or mortality but did have an effect on rate of recovered patients and hospital discharge [103]. Similarly, we found that four out of five studies comparing remdesivir to other treatments either failed to find significant differences in patient outcomes [36, 39, 51] or found unexpectedly opposing results between different remdesivir regimens and thus were inconclusive [34]. One placebo-controlled trial was stopped due to adverse events in patients treated with remdesivir [36]. Differences in findings may be due to different endpoints investigated or different levels of severity in patients, since the inclusion criteria varied between trials.

$\mathrm{LPV} / \mathrm{r}$ and umifenovir were initially recommended for treatment of COVID-19 in China [33, 94]. Early observational and randomized controlled studies of LPV/r failed to find a benefit with treatment [104]. A small systematic review that examined the efficacy and safety of lopinavir/ ritonavir in patients with COVID-19 found that lopinavir/ ritonavir did not significantly affect death, viral clearance, or "radiological improvement" when compared to other interventions [105]. Subsequent results obtained from two RCTs, RECOVERY [26] and DISCOVERY [37], provided strong evidence against the use of LPV/r for COVID-19, and there were no benefits with early $\mathrm{LPV} / \mathrm{r}$ treatment. Indeed, Arabi et al. reported that treatment with LPV/r led to worse outcomes compared to no antiviral treatment [40]. Thus, early administration of LPV/r or LPV/r use in patients with non-severe/non-critical forms of disease demonstrated little clinical value, and may be harmful.

The efficacy of umifenovir is unclear due to conflicting results obtained from relatively small studies. Of the four studies that included umifenovir in the study design [31, 47, 85], three studies failed to find a clinical benefit [31, 47, 85]. Moreover, early administration of umifenovir (median 6 days from symptom onset) did not influence the rate of positive-to-negative conversion of SARS-CoV-2 or rates of antipyresis, cough alleviation, or radiological findings of chest $\mathrm{CT}$ at days 7 or 14 after treatment [31]. In contrast, Nojomi et al. reported improvements in peripheral oxygen saturation, duration of hospitalization, need for ICU admission, white blood cell count, and erythrocyte sedimentation rate with umifenovir treatment as compared to LPV/r [31]. However, the time from symptom onset to treatment was not reported, and the group sizes were small $(n=50)$.

Similar to our study, Okoli et al. found that antivirals did not have an effect on either viral clearance or (allcause mortality) but unlike our conclusions, they also found that antivirals did not significantly improve clinical progression [5]. Additionally, Lai, Chao, and Hsueh's systematic review conclusions parallel ours as they found that remdesivir may increase time to clinical improvement and may be an effective treatment for mild and moderate COVID-19 and that sofosbuvir/daclatasvir may positively affect COVID-19 survival and clinical recovery [6]. However, their study does not include their methodology.

An important consideration when evaluating the efficacy of any drug, especially antivirals, is the state of disease course. Drugs that target viral replication, such as remdesivir, favipiravir, baloxavir marboxil, daclatasvir, and sofosbuvir, should be most effective if administered early in the viremic phase, as observed with other viruses (e.g. favipiravir treatment of Ebola) [106]. The SARS-CoV-2 viral load peaks within the first week of infection, which is earlier than that observed in SARSCoV-1 (10-14 days) and MERS-CoV (7-10 days) [93]. Two of the trials we reviewed found that administering remdesivir within 10 days of symptom onset led to better patient recovery outcomes [23,53]. Similarly, higher cumulative incidences of recovery were reported in moderate or severe COVID-19 patients treated with sofosbuvir/daclatasvir less than 8 days from symptom onset $[22,33]$. In contrast, no differences in clinical outcomes were observed with baloxavir marboxil or favipiravir [30] or $\mathrm{LPV} / \mathrm{r}$ when administered earlier relative to symptom onset. These data indicate that early administration of antiviral therapy may be critical to the efficacy of some COVID-19 treatments.

\section{Limitations}

There were several limitations noted in the included studies. Standard of care varied across studies and included or could have included other antiviral therapies. In these cases, attributing a treatment effect to a specific drug can be difficult. Drugs that are not approved for use as antivirals may have unconfirmed 
antiviral activity. Additionally, there are a number of drugs that possess little effect individually but can elevate the overall antiviral benefit when administered with other antivirals (eg, ribavirin). Thus, the magnitude of treatment effect for a given antiviral drug is uncertain. Studies were not screened based on severity of cases included, which likely accounts for some of the inconsistency in results. Also, 36 non-English articles were excluded, which may impact the conclusions. Finally, nine studies had group sizes of 40 subjects or less $[20,22,30,32,33,43,45,46,51]$, which may have resulted in insufficient statistical power and an increase in type II error (Additional file 2 and Additional file 3).

\section{Conclusions}

The design and implementation of RCTs is a time-consuming process that struggles to keep pace with the needs of clinicians during a pandemic. However, the high level of evidence obtained through sufficiently powered RCTs can provide confidence and/or clarification regarding results obtained from various observational studies. For antivirals that exhibit efficacy for COVID-19 treatment, early administration may be a critical factor in determining the quality of outcome. Larger studies are needed for antivirals that are less-described in COVID-19 treatment, such as sofosbuvir/daclatasvir, as these drugs may have equal or superior clinical outcomes compared to current therapies and may be more amenable for widespread use (ie, cheaper costs, oral availability).

\begin{abstract}
Abbreviations
ACTT-1: Adaptive Covid-19 Treatment Trial; Cl: Confidence interval; COVID19: Coronavirus disease 2019; CQ: Chloroquine; CT: Computed tomography; ECMO: Extracorporeal membrane oxygenation; Gl: Gastrointestinal; HCQ: Hydroxychloroquine; HR: Hazard ratio; ICU: Intensive care unit; IFN: Interferon; IQR: Interquartile range; LPV/r: Lopinavir/ritonavir; MERS: Middle Eastern respiratory syndrome; NMV: Noninvasive mechanical ventilation; OR: Odds ratio; PRISMA: Preferred Reporting Items for Systematic Reviews and Meta-Analyses; RCTs: Randomized control trials; RR: Rate ratio; RT-PCR: Reverse transcription polymerase chain reaction tests; $\mathrm{SaO}_{2}$ : Oxygen saturation; SARS: Severe acute respiratory syndrome; SARS-CoV-2: Severe acute respiratory syndrome coronavirus 2; SD: Standard deviation; SIGN: Scottish Intercollegiate Guidelines Network method; $\mathrm{SpO}_{2}$ : Oxygen saturation; UK: United Kingdom; WBC: White blood cell count.
\end{abstract}

\section{Supplementary Information}

The online version contains supplementary material available at https://doi. org/10.1186/s12879-022-07068-0.

Additional file 1: Table S1. Summary of risk of bias assessed with the Scottish Intercollegiate Guidelines Network (SIGN) randomized controlled trials checklist. Risk of bias assessment

Additional file 2. PRISMA checklist.

Additional file 3. PRISMA abstract checklist.

\section{Acknowledgements}

The authors acknowledge Karl Holub, Stephen Mead, Jeffrey Johnson, and Darian Lehmann-Plantenberg for their design and support of the Nested Knowledge meta-analytical software. The authors acknowledge Superior Medical Experts for their assistance in drafting and editing the manuscript.

\section{Authors' contributions}

CTRV: Conceptualization, Writing—Original Draft, Supervision. KE: Conceptualization, Writing — Original Draft, Visualization. H: Formal Analysis, Investigation, Data Curation, Visualization. IA: Formal Analysis, Investigation, Data Curation. AB: Formal Analysis, Investigation, Data Curation. NH: Conceptualization, Investigation, Data Curation, Writing — Review \& Editing, Supervision, Project Administration. BK: Writing—Original Draft, Writing—Review \& Editing, Visualization. PRK: Conceptualization, Methodology, Investigation, Writing-Original Draft. YSP: Writing—Original Draft, Visualization. ES: Conceptualization, Writing — Original Draft, Writing—Review \& Editing, Supervision, Project Administration. PB: Writing —Original Draft. RC: Conceptualization, Writing — Review \& Editing. SC: Writing — Original Draft, Visualization. KC: Data Curation, Supervision, Writing — Review \& Editing, Project Administration. JK: Formal Analysis, Investigation, Data Curation. LS: Formal Analysis, Investigation, Data Curation, Visualization. RT: Formal Analysis, Investigation, Data Curation, Visualization. CZ: Formal Analysis, Investigation, Data Curation. NG: Conceptualization, Methodology, Writing — Review \& Editing. KMK: Conceptualization, Resources, Supervision. KS: Conceptualization, Writing—Review \& Editing, Supervision. JT:Writing - Review \& Editing, Supervision. All authors read and approved the final manuscript.

\section{Funding}

This work was sponsored by Nested Knowledge, Inc. Employees of Nested Knowledge, Inc. performed study design, data collection, analysis, and interpretation and assisted in writing the manuscript as part of their employee duties.

\section{Availability of data and materials}

The datasets generated and/or analyzed during the current study are available in the Nested Knowledge website [9].

\section{Declarations}

Ethics approval and consent to participate

Not applicable.

\section{Consent for publication \\ Not applicable.}

\section{Competing interests}

JT is CEO and has ownership interest in Superior Medical Experts. ES and BK are employed by Superior Medical Experts. KE performed work on this project as an employee of Superior Medical Experts. KK is CEO of Nested Knowledge, Inc., has ownership interest in Nested Knowledge, Inc. and Superior Medical Experts, and consults for Medtronic. KC is employed by and has equity in Nested Knowledge. IZ, AB, CZ, NH, JK, HL, LS, and RT are employed by Nested Knowledge, Inc.

\section{Author details}

${ }^{1}$ Department of Internal Medicine, Jacobi Medical Center, Albert Einstein College of Medicine, 1400 Pelham Pkwy S, Bronx, NY, USA. ${ }^{2}$ Superior Medical Experts, 1425 Minnehaha Ave E, P.O. Box 6000545, St Paul, MN 55106, USA. ${ }^{3}$ Nested Knowledge, 1430 Avon Street N, Saint Paul, MN 55117, USA. ${ }^{4}$ Ohio University Heritage College of Osteopathic Medicine, 6775 Bobcat Way, Dublin, $\mathrm{OH} 43016$, USA. ${ }^{5}$ Kamineni Academy of Medical Sciences and Research Center, Hyderabad, Telangana 500068, India. ${ }^{6}$ Department of Medicine, Lakeridge Health, 1 Hospital Crt, Oshawa, ON L1G 2B9, Canada. ${ }^{7}$ Reading Hospital, 420 South 5th Avenue, West Reading, PA 19611, USA. ${ }^{8}$ Department of Infectious Disease, Kasturba Medical College, Manipal, Manipal Academy of Higher Education, Manipal, Karnataka 576104, India. ${ }^{9}$ Manipal Center for Infectious Diseases, Prasanna School of Public Health, Manipal Academy of Higher Education, Manipal, Karnataka 576104, India. ${ }^{10}$ Weill Cornell Medical College, 1300 York Ave, New York, NY 10065, USA. 
Received: 26 September 2021 Accepted: 14 January 2022

Published online: 31 January 2022

\section{References}

1. Weekly epidemiological update on COVID-19 - 14 December 2021. World Health Organization. https://www.who.int/publications/m/item/ weekly-epidemiological-update-on-covid-19---14-december-2021. Accessed 16 Dec 2021.

2. García LF. Immune response, inflammation, and the clinical spectrum of COVID-19. Front Immunol. 2020;11:1441.

3. Sanders JM, Monogue ML, Jodlowski TZ, Cutrell JB. Pharmacologic treatments for coronavirus disease 2019 (COVID-19): a review. JAMA 2020;323(18):1824-36.

4. Liu W, Zhou P, Chen K, Ye Z, Liu F, Li X, He N, Wu Z, Zhang Q, Gong $X$, et al. Efficacy and safety of antiviral treatment for COVID-19 from evidence in studies of SARS-CoV-2 and other acute viral infections: a systematic review and meta-analysis. CMAJ. 2020;192(27):E734-44.

5. Okoli GN, Rabbani R, Al-Juboori A, Copstein L, Askin N, Abou-Setta AM Antiviral drugs for coronavirus disease 2019 (COVID-19): a systematic review with network meta-analysis. Expert Rev Anti Infect Ther. 2021. https://doi.org/10.1080/14787210.2021.1961579.

6. Lai CC, Chao CM, Hsueh PR. Clinical efficacy of antiviral agents against coronavirus disease 2019: A systematic review of randomized controlled trials. J Microbiol Immunol Infect. 2021;54(5):767-75.

7. Nested Knowledge. https://nested-knowledge.com/. Accessed 31 Dec 2021.

8. Page MJ, McKenzie JE, Bossuyt PM, Boutron I, Hoffmann TC, Mulrow CD, Shamseer L, Tetzlaff JM, AkI EA, Brennan SE, et al. The PRISMA 2020 statement: an updated guideline for reporting systematic reviews. PLOS Med. 2021;18(3):e1003583.

9. COVID-19RCTs - Phase I. Nested Knowledge, Inc. https://nested-knowl edge.com/nest/qualitative/212. Accessed 14 Sep 2021.

10. Baloxavir Marboxil. U.S. Food and Drug Administration. https://www. accessdata.fda.gov/scripts/cder/daf/. Accessed 20 Dec 2021.

11. Lopinavir/Ritonavir. U.S. Food and Drug Administration. https://www. accessdata.fda.gov/scripts/cder/daf/. Accessed 20 Dec 2021.

12. Atazanavir. U.S. Food and Drug Administration. https://www.accessdata. fda.gov/scripts/cder/daf/. Accessed 20 Dec 2021

13. Sofosbuvir. U.S. Food and Drug Administration. https://www.acces sdata.fda.gov/scripts/cder/daf/. Accessed 20 Dec 2021.

14. Daclatasvir. U.S. Food and Drug Administration. https://www.acces sdata.fda.gov/scripts/cder/daf/. Accessed 20 Dec 2021.

15. Remdesivir. U.S. Food and Drug Administration. https://www.acces sdata.fda.gov/scripts/cder/daf/. Accessed 20 Dec 2021.

16. Ribavirin. U.S. Food and Drug Administration. https://www.accessdata. fda.gov/scripts/cder/daf/. Accessed 20 Dec 2021.

17. Shiraki K, Daikoku T. Favipiravir, an anti-influenza drug against lifethreatening RNA virus infections. Pharmacol Ther. 2020;209:107512.

18. Leneva IA, Russell RJ, Boriskin YS, Hay AJ. Characteristics of arbidolresistant mutants of influenza virus: implications for the mechanism of anti-influenza action of arbidol. Antiviral Res. 2009:81(2):132-40.

19. A Drug Safety and Dose-exploratory Clinical Study of Azvudine Tablets in Patients Who Have Not Received Anti-HIV Treatment. https://Clini calTrials.gov/show/NCT04109183. Accessed 20 Dec 2021.

20. Zheng F, Zhou Y, Zhou Z, Ye F, Huang B, Huang Y, Ma J, Zuo Q, Tan X, Xie J, et al. SARS-CoV-2 clearance in COVID-19 patients with Novaferon treatment: a randomized, open-label, parallel-group trial. Int J Infect Dis. 2020;99:84-91.

21. Methodology checklist 2: randomised controlled trials. https://www. sign.ac.uk/what-we-do/methodology/checklists/. Accessed $14 \mathrm{Sep}$ 2021.

22. Abbaspour Kasgari H, Moradi S, Shabani AM, Babamahmoodi F, Davoudi Badabi AR, Davoudi L, Alikhani A, Hedayatizadeh Omran A, Saeedi M, Merat S, et al. Evaluation of the efficacy of sofosbuvir plus daclatasvir in combination with ribavirin for hospitalized COVID-19 patients with moderate disease compared with standard care: a single-centre, randomized controlled trial. J Antimicrob Chemother. 2020;75(11):3373-8.
23. Beigel JH, Tomashek KM, Dodd LE, Mehta AK, Zingman BS, Kalil AC, Hohmann E, Chu HY, Luetkemeyer A, Kline S, et al. Remdesivir for the treatment of Covid-19-final report. N Engl J Med. 2020;383(19):1813-26

24. Cao B, Wang Y, Wen D, Liu W, Wang J, Fan G, Ruan L, Song B, Cai Y, Wei $M$, et al. A trial of lopinavir-ritonavir in adults hospitalized with severe Covid-19. N Engl J Med. 2020;382(19):1787-99.

25. Chen C, Zhang Y, Huang J, Yin P, Cheng Z, Wu J, Chen S, Zhang Y, Chen B, Lu M, et al. Favipiravir versus Arbidol for COVID-19: a randomized clinical trial. MedRxiv. 2020. https://doi.org/10.1101/2020.03.17.20037 432.

26. Group RC. Lopinavir-ritonavir in patients admitted to hospital with COVID-19 (RECOVERY): a randomised, controlled, open-label, platform trial. Lancet. 2020:396(10259):1345-52

27. Reis G, Moreira Silva E, Medeiros Silva DC, Thabane L, Singh G, Park $\mathrm{JJH}$, Forrest JI, Harari O, Quirino Dos Santos CV, Guimarães de Almeida APF et al. Effect of early treatment with hydroxychloroquine or Lopinavir and Ritonavir on risk of hospitalization among patients with COVID-19: The TOGETHER Randomized Clinical Trial. JAMA Netw Open. 2021:4(4):e216468.

28. Khalili H, Nourian A, Ahmadinejad Z, Emadi Kouchak H, Jafari S, Dehghan Manshadi SA, Rasolinejad M, Kebriaeezadeh A. Efficacy and safety of sofosbuvir/ledipasvir in treatment of patients with COVID-19; a randomized clinical trial. Acta Biomed. 2020;91(4):e2020102.

29. Li Y, Xie Z, Lin W, Cai W, Wen C, Guan Y, Mo X, Wang J, Wang Y, Peng P, et al. Efficacy and safety of lopinavir/ritonavir or arbidol in adult patients with mild/moderate COVID-19: an exploratory randomized controlled trial. Med (N Y). 2020; 1 (1):105-113.e104.

30. Lou Y, Liu L, Yao H, Hu X, Su J, Xu K, Luo R, Yang X, He L, Lu X, et al. Clinical outcomes and plasma concentrations of baloxavir marboxil and favipiravir in COVID-19 Patients: an exploratory randomized. Controlled Trial Eur J Pharm Sci. 2021;157:105631.

31. Nojomi M, Yassin Z, Keyvani H, Makiani MJ, Roham M, Laali A, Dehghan N, Navaei M, Ranjbar M. Effect of Arbidol (Umifenovir) on COVID-19: a randomized controlled trial. BMC Infect Dis. 2020;20(1):954.

32. Roozbeh F, Saeedi M, Alizadeh-Navaei R, Hedayatizadeh-Omran A, Merat S, Wentzel H, Levi J, Hill A, Shamshirian A. Sofosbuvir and daclatasvir for the treatment of COVID-19 outpatients: a double-blind, randomized controlled trial. J Antimicrob Chemother. 2021;76(3):753-7.

33. Sadeghi A, Ali Asgari A, Norouzi A, Kheiri Z, Anushirvani A, Montazeri M, Hosamirudsai H, Afhami S, Akbarpour E, Aliannejad R, et al. Sofosbuvir and daclatasvir compared with standard of care in the treatment of patients admitted to hospital with moderate or severe coronavirus infection (COVID-19): a randomized controlled trial. J Antimicrob Chemother. 2020;75(11):3379-85.

34. Spinner CD, Gottlieb RL, Criner GJ, Arribas López JR, Cattelan AM, Soriano Viladomiu A, Ogbuagu O, Malhotra P, Mullane KM, Castagna A, et al. Effect of remdesivir vs standard care on clinical status at 11 days in patients with moderate COVID-19: a randomized clinical trial. JAMA. 2020;324(11):1048-57.

35. Udwadia ZF, Singh P, Barkate H, Patil S, Rangwala S, Pendse A, Kadam J, Wu W, Caracta CF, Tandon M. Efficacy and safety of favipiravir, an oral RNA-dependent RNA polymerase inhibitor, in mild-to-moderate COVID-19: A randomized, comparative, open-label, multicenter, phase 3 clinical trial. Int J Infect Dis. 2021:103:62-71.

36. Wang Y, Zhang D, Du G, Du R, Zhao J, Jin Y, Fu S, Gao L, Cheng Z, Lu Q et al. Remdesivir in adults with severe COVID-19: a randomised, double-blind, placebo-controlled, multicentre trial. Lancet. 2020;395(10236):1569-78.

37. Ader F, Peiffer-Smadja N, Poissy J, Bouscambert-Duchamp M, Belhadi D, Diallo A, Delmas C, Saillard J, Dechanet A, Mercier N, et al. An open-labe randomized controlled trial of the effect of lopinavir/ritonavir, lopinavir/ ritonavir plus IFN- $\beta$ - $1 \mathrm{a}$ and hydroxychloroquine in hospitalized patients with COVID-19. Clin Microbiol Infect. 2021. https://doi.org/10.1016/j. cmi.2021.05.020.

38. Arabi YM, Gordon AC, Derde LPG, Nichol AD, Murthy S, Beidh FA, Annane D, Swaidan LA, Beane A, Beasley R, et al. Lopinavir-ritonavir and hydroxychloroquine for critically ill patients with COVID-19: REMAP-CAP randomized controlled trial. Intensive Care Med. 2021:47(8):867-86.

39. Barratt-Due A, Olsen IC, Nezvalova-Henriksen K, Kåsine T, LundJohansen F, Hoel H, Holten AR, Tveita A, Mathiessen A, Haugli M, et al. 
Evaluation of the effects of remdesivir and hydroxychloroquine on viral clearance in COVID-19: a randomized trial. Ann Intern Med. 2021. https://doi.org/10.7326/m21-0653.

40. Bosaeed M, Mahmoud E, Alharbi A, Altayib H, Albayat H, Alharbi F, Ghalilah K, Al Arfaj A, AlJishi J, Alarfaj A, et al. Favipiravir and hydroxychloroquine combination therapy in patients with moderate to severe COVID-19 (FACCT Trial): an open-label, multicenter, randomized. Controlled Trial Infect Dis Ther. 2021. https://doi.org/10.2139/ssrn.3829663.

41. Dabbous HM, Abd-Elsalam S, El-Sayed MH, Sherief AF, Ebeid FFS, El Ghafar MSA, Soliman S, Elbahnasawy M, Badawi R, Tageldin MA. Efficacy of favipiravir in COVID-19 treatment: a multi-center randomized study. Arch Virol. 2021;166(3):949-54

42. Dabbous HM, El-Sayed MH, El Assal G, Elghazaly H, Ebeid FFS, Sherief AF, Elgaafary M, Fawzy E, Hassany SM, Riad AR, et al. Safety and efficacy of favipiravir versus hydroxychloroquine in management of COVID-19: a randomised controlled trial. Sci Rep. 2021;11(1):7282.

43. Sayad B, Khodarahmi R, Najafi F, Miladi R, Mohseni Afshar Z, Mansouri F, Rahimi Z, Shirvani M, Salimi M, Vaziri S, et al. Efficacy and safety of sofosbuvir/velpatasvir versus the standard of care in adults hospitalized with COVID-19: a single-centre, randomized controlled trial. J Antimicrob Chemother. 2021;76(8):2158-67.

44. Solaymani-Dodaran M, Ghanei M, Bagheri M, Qazvini A, Vahedi E, Hassan Saadat S, Amin Setarehdan S, Ansarifar A, Biganeh H, Mohazzab A, et al. Safety and efficacy of Favipiravir in moderate to severe SARSCoV-2 pneumonia. Int Immunopharmacol. 2021;95:107522.

45. Zhao H, Zhang C, Zhu Q, Chen X, Chen G, Sun W, Xiao Z, Du W, Yao J, Li $\mathrm{G}$, et al. Favipiravir in the treatment of patients with SARS-CoV-2 RNA recurrent positive after discharge: a multicenter, open-label, randomized trial. Int Immunopharmacol. 2021;97:107702.

46. Abbass S, Kamal E, Salama M, Salman T, Sabry A, Abdel-Razek W, Helmy S, Abdelgwad A, Sakr N, Elgazzar M, et al. Efficacy and safety of sofosbuvir plus daclatasvir or ravidasvir in patients with COVID-19: A randomized controlled trial. J Med Virol. 2021. https://doi.org/10.1002/ jmv.27264.

47. Alavi Darazam I, Shokouhi S, Mardani M, Pourhoseingholi MA, Rabiei MM, Hatami F, Shabani M, Moradi O, Gharehbagh FJ, Irvani SSN, et al. Umifenovir in hospitalized moderate to severe COVID-19 patients: A randomized clinical trial. Int Immunopharmacol. 2021;99:107969.

48. Doi Y, Hibino M, Hase R, Yamamoto M, Kasamatsu Y, Hirose M, Mutoh Y, Homma Y, Terada M, Ogawa T, et al. A prospective, randomized, openlabel trial of early versus late favipiravir therapy in hospitalized patients with COVID-19. Antimicrob Agents Chemother. 2020. https://doi.org/ 10.1128/AAC.01897-20.

49. El-Bendary M, Abd-Elsalam S, Elbaz T, El-Akel W, Cordie A, Elhadidy T, Elalfy H, Farid K, Elegezy M, El-Badrawy A, et al. Efficacy of combined Sofosbuvir and Daclatasvir in the treatment of COVID-19 patients with pneumonia: a multicenter Egyptian study. Expert Rev Anti-infect Ther. 2021. https://doi.org/10.1080/14787210.2021.1950532.

50. Holubovska O, Bojkova D, Elli S, Bechtel M, Boltz D, Muzzio M, Peng X, Sala F, Cosentino C, Mironenko A, et al. Enisamium is an inhibitor of the SARS-CoV-2 RNA polymerase and shows improvement of recovery in COVID-19 patients in an interim analysis of a clinical trial. MedRxiv. 2021. https://doi.org/10.1101/2021.01.05.21249237.

51. Mahajan L, Singh AP, Gifty. Clinical outcomes of using remdesivir in patients with moderate to severe COVID-19: a prospective randomised study. Indian J Anaesth. 2021;65(Suppl 1):S41-S46.

52. Shinkai M, Tsushima K, Tanaka S, Hagiwara E, Tarumoto N, Kawada I, Hirai Y, Fujiwara S, Komase Y, Saraya T, et al. Efficacy and safety of favipiravir in moderate COVID-19 pneumonia patients without oxygen therapy: a randomized, phase III clinical trial. Infect Dis Ther. 2021. https://doi.org/10.1007/s40121-021-00517-41.

53. Goldman JD, Lye DCB, Hui DS, Marks KM, Bruno R, Montejano R, Spinner CD, Galli M, Ahn M-Y, Nahass RG, et al. Remdesivir for 5 or 10 Days in Patients with Severe Covid-19. N Engl J Med. 2020;383(19):1827-37.

54. Sekhavati E, Jafari F, SeyedAlinaghi S, Jamalimoghadamsiahkali S, Sadr S, Tabarestani M, Pirhayati M, Zendehdel A, Manafi N, Hajiabdolbaghi $M$, et al. Safety and effectiveness of azithromycin in patients with COVID-19: an open-label randomised trial. Int J Antimicrob Agents. 2020;56(4):106143.

55. Hung IF, Lung KC, Tso EY, Liu R, Chung TW, Chu MY, Ng YY, Lo J, Chan J, Tam AR, et al. Triple combination of interferon beta- $1 \mathrm{~b}$, lopinavir-ritonavir, and ribavirin in the treatment of patients admitted to hospital with COVID-19: an open-label, randomised, phase 2 trial. Lancet. 2020;395(10238):1695-704.

56. Zhao H, Zhu Q, Zhang C, Li J, Wei M, Qin Y, Chen G, Wang K, Yu J, Wu Z, et al. Tocilizumab combined with favipiravir in the treatment of COVID19: a multicenter trial in a small sample size. Biomed Pharmacother. 2021;133:110825.

57. Zheng F, Zhou Y, Zhou Z, Ye F, Huang B, Huang Y, Ma J, Zuo Q, Tan X, Xie $J$, et al. A novel protein drug, novaferon, as the potential antiviral drug for COVID-19. MedRxiv. 2020;395:565.

58. Khamis F, Al Naabi H, Al Lawati A, Ambusaidi Z, Al Sharji M, Al Barwani U, Pandak N, Al Balushi Z, Al Bahrani M, Al Salmi I, et al. Randomized controlled open label trial on the use of favipiravir combined with inhaled interferon beta- $1 \mathrm{~b}$ in hospitalized patients with moderate to severe COVID-19 pneumonia. Int J Infect Dis. 2021;102:538-43.

59. Okumuş N, Demirtürk N, Çetinkaya RA, Güner R, Avcı IY, Orhan S, Konya P, Şaylan B, Karalezli A, Yamanel L, et al. Evaluation of the effectiveness and safety of adding ivermectin to treatment in severe COVID-19 patients. BMC Infect Dis. 2021;21(1):411.

60. Mahmud R, Rahman MM, Alam I, Ahmed KGU, Kabir A, Sayeed S, Rassel MA, Monayem FB, Islam MS, Islam MM, et al. Ivermectin in combination with doxycycline for treating COVID-19 symptoms: a randomized trial. J Int Med Res. 2021;49(5):3000605211013550.

61. Group PTC. Azithromycin for community treatment of suspected COVID-19 in people at increased risk of an adverse clinical course in the UK (PRINCIPLE): a randomised, controlled, open-label, adaptive platform trial. Lancet. 2021;397(10279):1063-74.

62. López-Medina E, López P, Hurtado IC, Dávalos DM, Ramirez O, Martínez E, Díazgranados JA, Oñate JM, Chavarriaga H, Herrera S, et al. Effect of ivermectin on time to resolution of symptoms among adults with mild COVID-19: a randomized clinical trial. JAMA. 2021;325(14):1426-35.

63. Group RC. Azithromycin in patients admitted to hospital with COVID19 (RECOVERY): a randomised, controlled, open-label, platform trial. Lancet. 2021;397(10274):605-12.

64. Ahmed S, Karim MM, Ross AG, Hossain MS, Clemens JD, Sumiya MK, Phru CS, Rahman M, Zaman K, Somani J, et al. A five-day course of ivermectin for the treatment of COVID-19 may reduce the duration of illness. Int J Infect Dis. 2021;103:214-6.

65. Omrani AS, Pathan SA, Thomas SA, Harris TRE, Coyle PV, Thomas CE, Qureshi I, Bhutta ZA, Mawlawi NA, Kahlout RA, et al. Randomized double-blinded placebo-controlled trial of hydroxychloroquine with or without azithromycin for virologic cure of non-severe Covid-19. EClinicalMedicine. 2020;29:100645.

66. Furtado RHM, Berwanger O, Fonseca HA, Corrêa TD, Ferraz LR, Lapa MG, Zampieri FG, Veiga VC, Azevedo LCP, Rosa RG, et al. Azithromycin in addition to standard of care versus standard of care alone in the treatment of patients admitted to the hospital with severe COVID-19 in Brazil (COALITION II): a randomised clinical trial. Lancet. 2020;396(10256):959-67.

67. Brown SM, Peltan I, Kumar N, Leither L, Webb BJ, Starr N, Grissom CK, Buckel WR, Srivastava R, Butler AM, et al. Hydroxychloroquine vs. azithromycin for hospitalized patients with COVID-19 (HAHPS): results of a randomized, active comparator trial. Ann Am Thorac Soc. 2020;18(4):590-7.

68. Purwati, Budiono, Rachman BE, Yulistiani, Miatmoko A, Nasronudin, Lardo S, Purnama YI, Laely M, Rochmad I, et al. A randomized, doubleblind, multicenter clinical study comparing the efficacy and safety of a drug combination of lopinavir/ritonavir-azithromycin, lopinavir/ritonavir-doxycycline, and azithromycin-hydroxychloroquine for patients diagnosed with mild to moderate COVID-19 infections. Biochem Res Int. 2021;2021:6685921.

69. Gupta S, Dixit PK, Ghana P, Abhisheka K, Khurana H, Jha VK, Mahapatra D, Goel J, Ahmed S, Varadaraj G. Open-label randomized control trial of hydroxychloroquine in patients with moderate to severe coronavirus disease 2019 infection. Med J Armed Forces India. 2021;77(Suppl 2):S305-s311.

70. Rahmani H, Davoudi-Monfared E, Nourian A, Khalili H, Hajizadeh N, Jalalabadi NZ, Fazeli MR, Ghazaeian M, Yekaninejad MS. Interferon $\beta-1 b$ in treatment of severe COVID-19: a randomized clinical trial. Int Immunopharmacol. 2020;88:106903. 
71. Vlaar APJ, de Bruin S, Busch M, Timmermans S, van Zeggeren IE, Koning $R$, Ter Horst L, Bulle EB, van Baarle F, van de Poll MCG, et al. AntiC5a antibody IFX-1 (vilobelimab) treatment versus best supportive care for patients with severe COVID-19 (PANAMO): an exploratory, open-label, phase 2 randomised controlled trial. Lancet Rheumatol. 2020;2(12):e764-73.

72. Wu X, Yu K, Wang Y, Xu W, Ma H, Hou Y, Li Y, Cai B, Zhu L, Zhang M, et al. Efficacy and safety of triazavirin therapy for coronavirus disease 2019: a pilot randomized controlled trial. Engineering (Beijing). 2020;6(10):1185-91.

73. Horby P, Mafham M, Linsell L, Bell JL, Staplin N, Emberson JR, Wiselka M, Ustianowski A, Elmahi E, Prudon B, et al. Effect of Hydroxychloroquine in Hospitalized Patients with Covid-19. N Engl J Med. 2020;383(21):2030-40.

74. Abella BS, Jolkovsky EL, Biney BT, Uspal JE, Hyman MC, Frank I, Hensley SE, Gill S, Vogl DT, Maillard I, et al. Efficacy and safety of hydroxychloroquine vs placebo for pre-exposure SARS-CoV-2 prophylaxis among health care workers: a randomized clinical trial. JAMA Intern Med. 2021;181(2):195-202.

75. Alavi Darazam I, Shokouhi S, Pourhoseingholi MA, Naghibi Irvani SS, Mokhtari M, Shabani M, Amirdosara M, Torabinavid P, Golmohammadi M, Hashemi S, et al. Role of interferon therapy in severe COVID-19: the COVIFERON randomized controlled trial. Sci Rep. 2021;11(1):8059.

76. Li C, Luo F, Liu C, Xiong N, Xu Z, Zhang W, Yang M, Wang Y, Liu D, Yu $C$, et al. Effect of a genetically engineered interferon-alpha versus traditional interferon-alpha in the treatment of moderate-to-severe COVID-19: a randomised clinical trial. Ann Med. 2021;53(1):391-401.

77. Kalil AC, Patterson TF, Mehta AK, Tomashek KM, Wolfe CR, Ghazaryan V, Marconi VC, Ruiz-Palacios GM, Hsieh L, Kline S, et al. Baricitinib plus remdesivir for hospitalized adults with Covid-19. N Engl J Med. 2021;384(9):795-807.

78. Johnston C, Brown ER, Stewart J, Karita HCS, Kissinger PJ, Dwyer J, Hosek S, Oyedele T, Paasche-Orlow MK, Paolino K, et al. Hydroxychloroquine with or without azithromycin for treatment of early SARS CoV-2 infection among high-risk outpatient adults: a randomized clinical trial. EClinicalMedicine. 2021;33:100773.

79. Seet RCS, Quek AML, Ooi DSQ, Sengupta S, Lakshminarasappa SR, Koo CY, So JBY, Goh BC, Loh KS, Fisher D, et al. Positive impact of oral hydroxychloroquine and povidone-iodine throat spray for COVID-19 prophylaxis: an open-label randomized trial. Int J Infect Dis. 2021:106:314-22

80. Kestelyn E, Dung NTP, Lam Minh Y, Hung LM, Quan NM, Dung NT, Minh NNQ, Xuan TC, Phong NT, Ninh Thi Thanh V, et al. A multi centre randomized open label trial of chloroquine for the treatment of adults with SARS-CoV-2 infection in Vietnam. Wellcome Open Res. 2020:5:141

81. Les Bujanda I, Loureiro-Amigo J, Bastons FC, Guerra IE, Sánchez JA, Murgadella-Sancho A, Rey RG, López JL, Álvarez JS. Treatment of COVID-19 pneumonia with glucocorticoids (CORTIVID): a structured summary of a study protocol for a randomised controlled trial. Trials. 2021;22(1):43.

82. Duška F, Waldauf $\mathrm{P}$, Halačová M, Zvoníček V, Bala J, Balík M, Beneš J, Klementová O, Kozáková I, Kubricht V, et al. Azithromycin added to hydroxychloroquine for patients admitted to intensive care due to coronavirus disease 2019 (COVID-19)-protocol of randomised controlled trial AZIQUINE-ICU. Trials. 2020;21(1):631.

83. Bosaeed M, Alharbi A, Hussein M, Abalkhail M, Sultana K, Musattat A, Alqahtani H, Alshamrani M, Mahmoud E, Alothman A, et al. Multicentre randomised double-blinded placebo-controlled trial of favipiravir in adults with mild COVID-19. BMJ Open. 2021;11(4):e047495.

84. Furuta Y, Komeno T, Nakamura T. Favipiravir (T-705), a broad spectrum inhibitor of viral RNA polymerase. Proc J.jnn Acad Ser B Phys Biol Sci. 2017:93(7):449-63.

85. Wang S, Dong K, Chen W. Development of a hemoptysis risk prediction model for patients following CT-guided transthoracic lung biopsy. BMC Pulm Med. 2020;20(1):247.

86. Vankadari N. Arbidol: A potential antiviral drug for the treatment of SARS-CoV-2 by blocking trimerization of the spike glycoprotein. Int J Antimicrob Agents. 2020;56(2):105998.

87. Mangum EM, Graham KK. Lopinavir-Ritonavir: a new protease inhibitor. Pharmacotherapy. 2001;21(11):1352-63.
88. Tanabe Y, Sakamoto N, Enomoto N, Kurosaki M, Ueda E, Maekawa S, Yamashiro T, Nakagawa M, Chen CH, Kanazawa N, et al. Synergistic inhibition of intracellular hepatitis C virus replication by combination of ribavirin and interferon- alpha. J Infect Dis. 2004;189(7):1129-39.

89. Chu CM, Cheng VC, Hung IF, Wong MM, Chan KH, Chan KS, Kao RY, Poon LL, Wong CL, Guan Y, et al. Role of lopinavir/ritonavir in the treatment of SARS: initial virological and clinical findings. Thorax. 2004;59(3):252-6.

90. Kiselev OI, Maleev WV, Deeva EG, Leneva IA, Selkova EP, Osipova EA, Obukhov AA, Nadorov SA, Kulikova EV. Clinical efficacy of arbidol (umifenovir) in the therapy of influenza in adults: preliminary results of the multicenter double-blind randomized placebo-controlled study ARBITR. Ter Arkh. 2015;87(1):88-96.

91. Spanakis N, Tsiodras S, Haagmans BL, Raj VS, Pontikis K, Koutsoukou A, Koulouris NG, Osterhaus AD, Koopmans MP, Tsakris A. Virological and serological analysis of a recent Middle East respiratory syndrome coronavirus infection case on a triple combination antiviral regimen. Int J Antimicrob Agents. 2014;44(6):528-32.

92. Sheahan TP, Sims AC, Leist SR, Schäfer A, Won J, Brown AJ, Montgomery SA, Hogg A, Babusis D, Clarke MO, et al. Comparative therapeutic efficacy of remdesivir and combination lopinavir, ritonavir, and interferon beta against MERS-CoV. Nat Commun. 2020;1 1(1):222.

93. Cevik M, Tate M, Lloyd O, Maraolo AE, Schafers J, Ho A. SARS-CoV-2, SARS-CoV, and MERS-CoV viral load dynamics, duration of viral shedding, and infectiousness: a systematic review and meta-analysis. Lancet Microbe. 2021;2(1):e13-22.

94. Diagnosis and treatment of pneumonia caused by new coronavirus (trial version 6). Medical Administration and Hospital Authority. www. nhc.gov.cn/yzygj/s7653p/202002/8334a8326dd94d329df351d7da8aef c2.shtml. Accessed 28 Apr 2021.

95. Bhatia HK, Singh H, Grewal N, Natt NK. Sofosbuvir: A novel treatment option for chronic hepatitis C infection. J Pharmacol Pharmacother. 2014;5(4):278-84.

96. Chien M, Anderson TK, Jockusch S, Tao C, Li X, Kumar S, Russo JJ, Kirchdoerfer RN, Ju J. Nucleotide analogues as inhibitors of SARSCoV-2 polymerase, a key drug target for COVID-19. J Proteome Res. 2020;19(11):4690-7.

97. Elfiky AA. Ribavirin, remdesivir, sofosbuvir, galidesivir, and tenofovir against SARS-CoV-2 RNA dependent RNA polymerase (RdRp): a molecular docking study. Life Sci. 2020;253:117592.

98. Walker AP, Fan H, Keown JR, Margitich V, Grimes JM, Fodor E, Te Velthuis AJW. Enisamium is a small molecule inhibitor of the influenza A virus and SARS-CoV-2 RNA polymerases. BioRxiv. 2020. https://doi.org/10. 1101/2020.04.21.053017.

99. Hill A, Wang J, Levi J, Heath K, Fortunak J. Minimum costs to manufacture new treatments for COVID-19. J Virus Erad. 2020;6(2):61-9.

100. Merat S. SD1000: high sustained viral response rate in 1361 patients with hepatitis C genotypes 1, 2, 3, and 4 using a low-cost, fixed-dose combination tablet of generic sofosbuvir and daclatasvir: a multicenter, phase III clinical trial. Clin Infect Dis. 2020;70(10):2206-12.

101. Poustchi H, Majd Jabbari S, Merat S, Sharifi AH, Shayesteh AA, Shayesteh E, Minakari M, Fattahi MR, Moini M, Roozbeh F, et al. The combination of sofosbuvir and daclatasvir is effective and safe in treating patients with hepatitis $C$ and severe renal impairment. J Gastroenterol Hepatol. 2020;35(9):1590-4.

102. Reddy Vegivinti CT, Pederson JM, Saravu K, Gupta N, Barrett A, Davis AR, Kallmes KM, Evanson KW. Remdesivir therapy in patients with COVID19: A systematic review and meta-analysis of randomized controlled trials. Ann Med Surg (Lond). 2021;62:43-8.

103. Al-Abdouh A, Bizanti A, Barbarawi M, Jabri A, Kumar A, Fashanu OE, Khan SU, Zhao D, Antar AAR, Michos ED. Remdesivir for the treatment of COVID-19: a systematic review and meta-analysis of randomized controlled trials. Contemp Clin Trials. 2021;101:106272.

104. Joseph BA, Dibas M, Evanson KW, Paranjape G, Vegivinti CTR, Selvan PT, Saravu K, Gupta N, Pulakurthi YS, Keesari PR, et al. Efficacy and safety of lopinavir/ritonavir in the treatment of COVID-19: a systematic review. Expert Rev Anti Infect Ther. 2021;19(6):679-87.

105. Patel TK, Patel PB, Barvaliya M, Saurabh MK, Bhalla HL, Khosla P. Efficacy and safety of lopinavir-ritonavir in COVID-19: a systematic review of randomized controlled trials. J Infect Public Health. 2021;14(6):740-8. 
106. Madelain V, Oestereich L, Graw F, Nguyen TH, de Lamballerie X, Mentré F, Günther S, Guedj J. Ebola virus dynamics in mice treated with favipiravir. Antiviral Res. 2015;123:70-7.

\section{Publisher's Note}

Springer Nature remains neutral with regard to jurisdictional claims in published maps and institutional affiliations.

- fast, convenient online submission

- thorough peer review by experienced researchers in your field

- rapid publication on acceptance

- support for research data, including large and complex data types

- gold Open Access which fosters wider collaboration and increased citations

- maximum visibility for your research: over $100 \mathrm{M}$ website views per year

At BMC, research is always in progress.

Learn more biomedcentral.com/submissions 